\title{
MOLECULARLY IMPRINTED POLYMERS AND THEIR APPLICATION AS ENVIRONMENTAL SENSORS
}

A Dissertation
presented to
the Faculty of the Graduate School
at the University of Missouri-Columbia
In Partial Fulfillment
of the Requirements for the Degree
Doctor of Philosophy
by
JINGJING DAI
Dr. Maria Fidalgo, Dissertation Supervisor
December 2017


The undersigned, appointed by the dean of the Graduate School, have examined the dissertation entitled

\section{MOLECULARLY IMPRINTED POLYMERS AND THEIR APPLICATION AS ENVIRONMENTAL SENSORS}

presented by Jingjing Dai, a candidate for the degree of doctor of philosophy, and hereby certify that, in their opinion, it is worthy of acceptance.

Professor Maria Fidalgo

Professor Baolin Deng

Professor Enos Inniss

Professor Chung-Ho Lin 


\section{DEDICATION}

\section{To Julian}

I wish a better world for you. We will have clean water, clean air for everyone on the earth. We will end poverty and reach sustainability, equality, and harmony. 


\section{ACKNOWLEDGEMENTS}

I would like to sincerely thank my dissertation advisor Dr. Maria Fidalgo, who gave me the great opportunity to do my $\mathrm{PhD}$ research along with her continuous support and motivation. She has been and will always be my advisor and mentor.

I also would like to express my gratitude and appreciation to all the members in my committee, Dr. Baolin Deng, Dr. Enos Innis, and Dr. Chung-Ho Lin, who have been there with me for the past three years providing their knowledge, experience, inspirations and encouragement.

Furthermore, my thanks go to all my collaborators Dr. Xiaoqing Dong, Dr. Susan Nagal, Dr. Chung-Ho Lin, Bayati Mohamed, and Danh Vu, for their advices and expertise.

Finally, I would like to thank all the group members, colleagues, professors and staff in the department for their help and friendship. You all helped make this possible. 


\section{Table of Contents}

ACKNOWLEDGEMENTS ................................................................................. ii

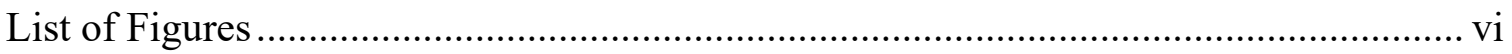

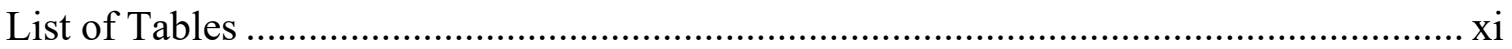

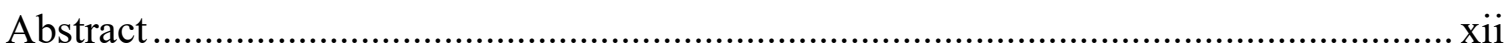

Chapter 1. Background and Introduction.............................................................. 1

1.1. Carbon Dots ...................................................................................... 3

1.1.1. Functionalized Carbon Dots ......................................................... 5

1.1.2. Fluorescence properties and Applications ...................................... 7

1.2. Molecularly Imprinted Polymers …................................................... 7

1.2.1. MIPs and their applications as environmental sensors ..................... 10

1.2.2. Characterization methods of MIPs.............................................. 12

1.2.3. Stober method ......................................................................... 16

1.3. DNT and its Environmental Sensors....................................................... 18

1.3.1. DNT and its retarding effects on vinyl polymerization .................... 18

1.3.2. DNT sensors.......................................................................... 19

1.3.3. The effects of water chemistry and interferences ........................... 20

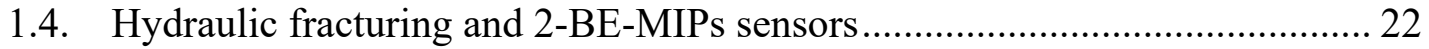

1.4.1. Hydraulic fracturing and its fingerprinting contaminants................. 22

1.4.2. UV-vis reflectance and 2BE MIP sensors ................................... 25

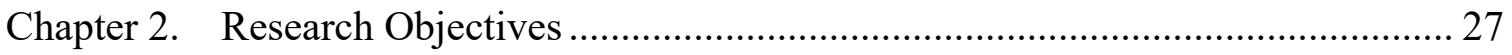

Chapter 3. Effect of water chemistry on the aggregation and photoluminescence

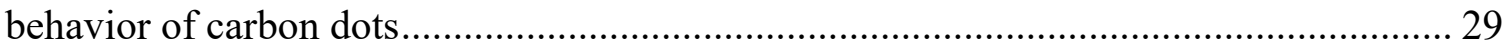




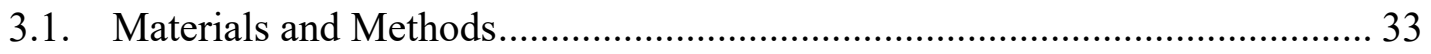

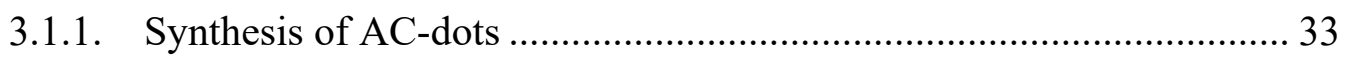

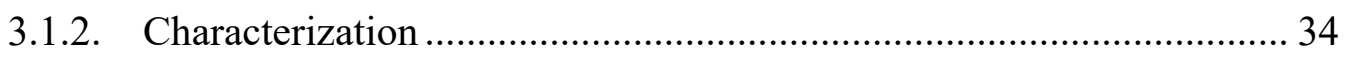

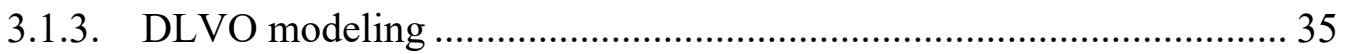

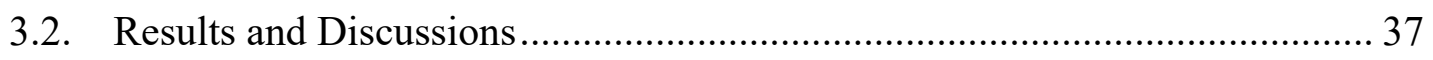

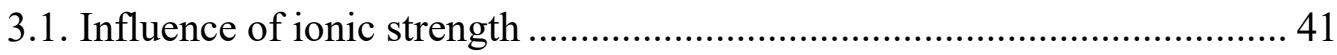

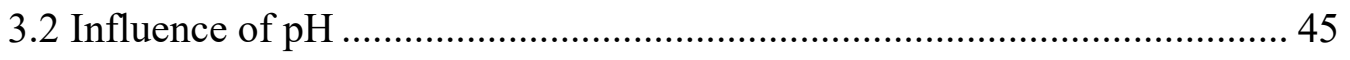

3.3 Influence of Natural Organic Matter......................................................... 48

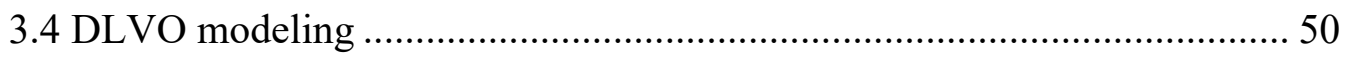

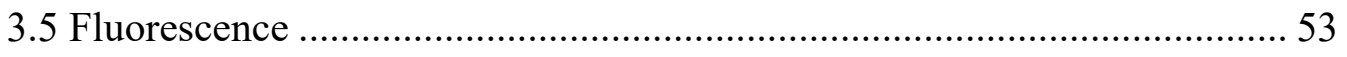

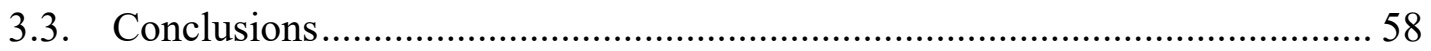

Chapter 4. Molecularly imprinted polymers labeled with amino-functionalized carbon dots for fluorescent determination of 2,4-dinitrotoluene .............................................. 60

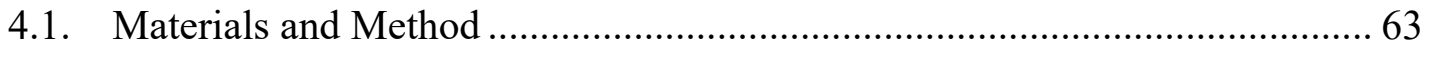

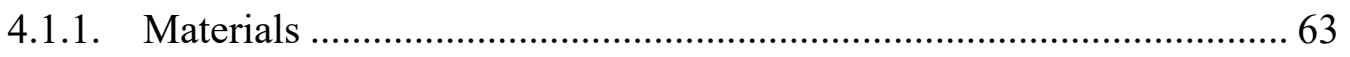

4.1.2. Synthesis of the colloidal crystals and MIPs ....................................... 63

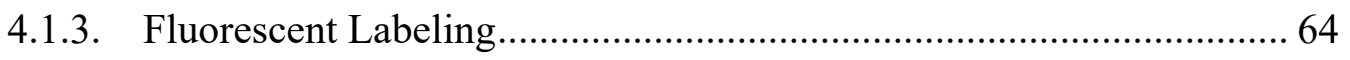

4.1.4. Characterization of NIP-PAA and NIP-PMAAA ................................ 65

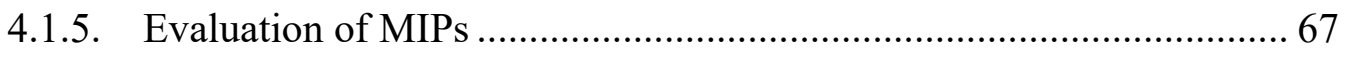

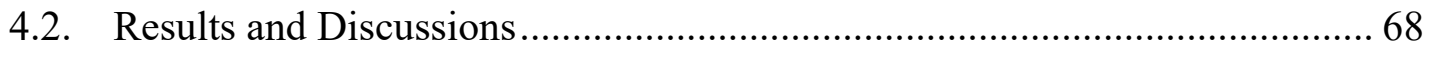

4.2.1. Preparation and Characterization of AC-dots and Porous MIPs .......... 68

4.2.2. Recognition Capacities of MIPs, NIP-PMAAA, and NIP-PAA.......... 73

4.2.3. Photoluminescence Properties of MIP Sensors .................................... 78 
4.2.4. Selectivity of MIP sensors ……………………............................... 82

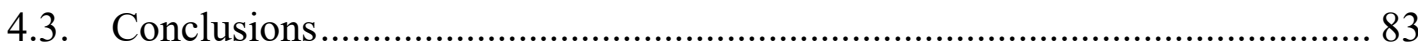

Chapter 5. Effect of $\mathrm{pH}$, ionic strength and natural organic matter on the DNT-MIP

fluorescent sensor

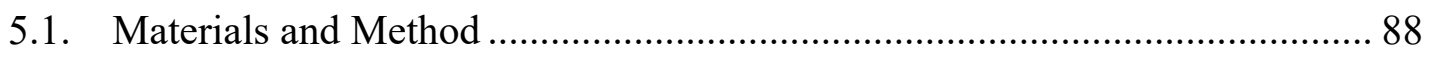

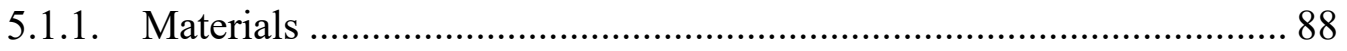

5.1.2. Synthesis of MIPs and Fluorescent labeling ...................................... 89

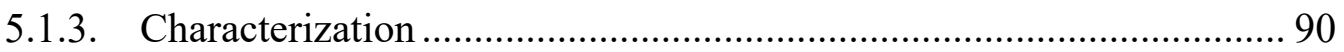

5.1.4. Fluorescence Quenching Experiments................................................... 91

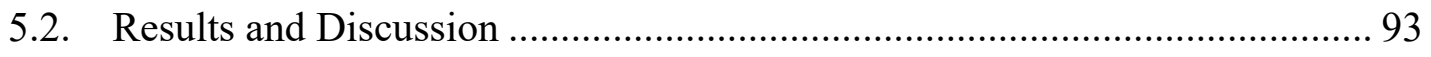

5.2.1. Characterization Results ................................................................. 93

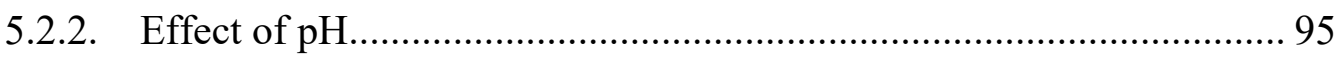

5.2.3. Effect of Ionic Strength and Different Salts Types............................. 98

5.2.4. Effect of NOM on Fluorescent Quenching .......................................... 99

5.2.5. Analytical applications in natural water samples............................... 102

5.3. Conclusion and Future Study ..................................................................... 104

Chapter 6. Water Quality Monitoring at Hydraulic Fracturing Sites Using Molecularly Imprinted Porous Hydrogels .................................................................................... 105

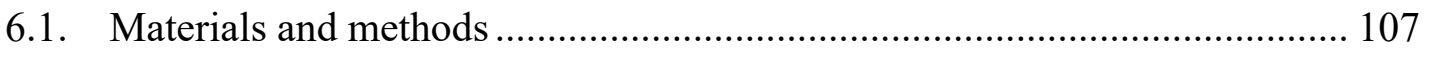

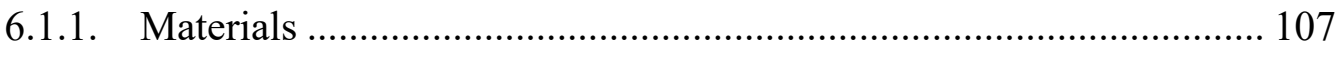

6.1.2. Molecularly Imprinted Porous Films Fabrication................................. 107

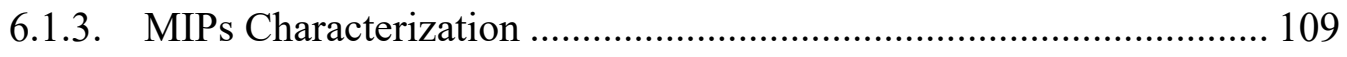

6.1.4. Binding / Incubation tests ................................................................ 110 
6.1.5. Optical sensing tests.............................................................. 112

6.2. Results and Discussion ...................................................................... 113

6.2.1. Characterization of MIPs ..................................................... 113

6.2.2. Recognition Capacities of MIPs and NIPs.................................. 116

6.2.3. Sensing properties of MIPs ..................................................... 117

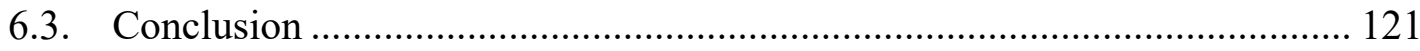

Chapter 7. Conclusions and Future Study........................................................... 123

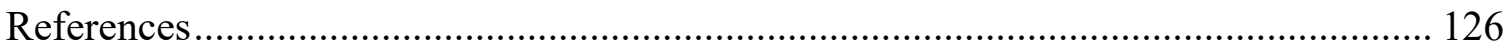

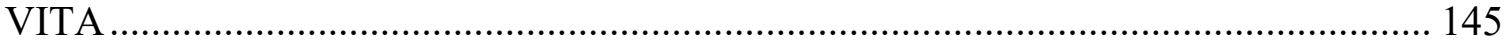




\section{List of Figures}

Figure 1.1 A polymer matrix is formed through polymerization by functional monomers and template. Molecularly imprinted polymers are obtained by

removing templates with cavities left

Figure 1.2 Batch rebinding approach is accomplished by serial incubation and then the polymer is filtrated to determine the free template in each vessel (GarcíaCalzón and Díaz-García 2007) 12

Figure 1.3 Different topologies of polymers can be linear, branched, macroscopic network and microgel, which is important in imprinting effects of MIPs (Cormack and Elorza 2004).

Figure 1.4 Schematic illustration of binding site homogeneity and heterogeneity in MIPs (Umpleby et al. 2004) 16

Figure 1.5 Radicals attack on the ring of nitroaromatic compounds 19

Figure 1.6 Radicals attack on the nitro groups of nitroaromatic compounds 19

Figure 3.1 (a), (c) Representative TEM image of a-CDs and CDs: insets represent the segmented particles after subtracting the background using ImageJ software.

(b), (d) Size distribution of a-CDs and CDs, as percent number of particles. 38

Figure 3.2 (a), (b) Representative HRTEM images of a-CDs and CDs respectively with lattice parameters of $0.32 \mathrm{~nm}$. 39

Figure 3.3 FT-IR spectra of: (a) a-CDs. (b) CDs 40

Figure 3.4 Zeta potential of $\mathrm{CDs}(\mathrm{pH}=5.7)$ and a-CDs $(\mathrm{pH}=9.7)$ as a function of ionic strength $\left(\mathrm{NaCl}\right.$ and $\left.\mathrm{CaCl}_{2}\right)$ 43 
Figure 3.5 Hydrodynamic diameter of $\mathrm{CDs}(\mathrm{pH}=5.7)$, a-dots $(\mathrm{pH}=9.7)$ as a function of ionic strength $\left(\mathrm{NaCl}\right.$ and $\left.\mathrm{CaCl}_{2}\right)$...... 43

Figure 3.6 Zeta potential of CDs, a-CDs as a function of $\mathrm{pH}$. (a) $1 \mathrm{mM}$ ionic strength given by $\mathrm{NaCl}$. (b) $1 \mathrm{mM}$ ionic strength given by $\mathrm{CaCl}_{2}$. 46

Figure 3.7 Hydrodynamic diameter of a-CDs, CDs as a function of pH. (a) $1 \mathrm{mM}$ ionic strength given by $\mathrm{NaCl}$. (b) $1 \mathrm{mM}$ ionic strength given by $\mathrm{CaCl}_{2}$ 48

Figure 3.8 Hydrodynamic diameter (a) and zeta potential (b) of CDs, a-CDs suspended in humic acid solutions of variable concentration at neutral $\mathrm{pH}$. 50

Figure 3.9 Calculated DLVO interaction energy plotted as a function of separation distance between CDs particles; effect of ionic strength and $\mathrm{pH}$ variations. (a) CDs with different $\mathrm{NaCl}$ concentrations. (b) $\mathrm{CDs}$ with different $\mathrm{CaCl}_{2}$ concentrations. (c) CDs in $1 \mathrm{mM} \mathrm{NaCl}$ with different $\mathrm{pH}$. (d) CDs in $1 \mathrm{mM}$ $\mathrm{CaCl}_{2}$ with different $\mathrm{pH}$.

Figure 3.10 Calculated DLVO interaction energy plotted as a function of separation distance between a-CDs particles; effect of ionic strength and $\mathrm{pH}$ variations. (a) a-CDs with different $\mathrm{NaCl}$ concentrations. (b) a-CDs with different $\mathrm{CaCl}_{2}$ concentrations. (c) a-CDs in $1 \mathrm{mM} \mathrm{NaCl}$ with different $\mathrm{pH}$. (d) a-CDs in $1 \mathrm{mM}$ $\mathrm{CaCl}_{2}$ with different $\mathrm{pH}$. 52

Figure 3.11 Fluorescence spectra for (a) CDs, and (b) a-CDs, at different concentrations 54

Figure 3.12 Fluorescence spectra for (a) CDs, and (b) a-CDs, at various levels of ionic strength and electrolytes. 
Figure 3.13 Absorption and fluorescence spectra: CDs (a) and (b); a-CDs(c) and (d), respectively, in the presence of HA; comparison of fluorescence intensity peak for CDs, a-CDs suspended in HA solutions (1ppm-100ppm) and pure HA (e).

Figure 4.1 TEM image of AC-Dots (a); (b) represents the segmented particles after subtracting the background by ImageJ software. 68

Figure 4.2 FTIR spectrum of AC-dots 69

Figure 4.3 Fluorescence spectra of A-CDs at different $\mathrm{pH}$, and inset graph shows

plot of FL peak vs. $\mathrm{pH}$ 70

Figure 4.4 Zeta potential of AC-Dots at different $\mathrm{pH}$ 70

Figure 4.5 Fluorescence spectra of AC-dots with increasing concentrations of DNT, and inset graph shows FL intensity vs. DNT concentrations. 71

Figure 4.7 Fluorescence spectra of Copolymer (a) and AC-Dots labeled Copolymer

(b) 73

Figure 4.8 Recognition Capacities of label-wash films (L-W), wash-label films (W-

L), and non-labeled films (NL) at DNT concentration of $10 \mathrm{ppm}$. 73

Figure 4.9 Recognition capacities of NIP-PMAAA, NIP-PAA and MIP. 74

Figure 4.10 Swelling ratio \% of nonporous PAA (open) and PMAAA films (hatched) 75

Figure 4.11 DSC thermograms of NIP-Copolymer (a) and NIP-PAA (b)....................... 76

Figure 4.12 FTIR spectra of NIP-PMAAA (a) and NIP-PAA (b) ............................... 77

Figure 4.13 ${ }^{1} \mathrm{H}$ NMR spectra of NIP-Copolymer (A) and NIP-PAA (B) ........................ 78 
Figure 4.14 Fluorescence emission spectra of C-MIPs with an increasing concentration of DNT (a) and Stern-Volmer plot of C-MIPs with an increasing concentration of DNT (b)

Figure 4.15 Fluorescence response time of C-MIP for DNT and adsorption kinetics of MIP with initial concentration of $10 \mathrm{ppm}$

Figure 4.16 The cycle curves of the AC-Dots labeled MIPs in the absence (the upper dots) and the presence (the bottom dots) of $10 \mathrm{ppm}$ DNT.

Figure 4.17 Quenching amount of DNT in presence of analogues; [DNT] $=0.04 \mathrm{mM}$; [analogues] $=0.02 \mathrm{mM}$

Figure 5.1 Swelling ratios of PMAAA films 95

Figure 5.2. Swelling Ratio\% of MIP films at different ionic strength with different salts 95

Figure 5.3 Fluorescence intensity of AC-Dots response to $\mathrm{pHs}$ 97

Figure 5.4 FL Intensity of MIP sensors measured in acidic $(\mathrm{pH}=4)$ and basic $(\mathrm{pH}=10)$ environments and measured after rinse in the absence of DNT

Figure 5.5 Quenching by DNT (10 ppm) at different $\mathrm{pHs}$ 97

Figure 5.6 Quenching by DNT (10 ppm) with different salts, $\mathrm{pH}=6$ 99

Figure 5.7 Quenching of AC-Dots and AC-Dots labeled MIPs by variable concentrations of SRNOM, in absence of DNT. 100

Figure 5.8 Quenching by DNT (10 ppm) with Suwannee River NOM 101

Figure 5.9 Stern-Volmer plot of AC-MIPs with an increasing concentration of DNT 102

Figure 6.1 Schematic of the MIP fabrication process: (a) monomer infiltration of the colloidal silica deposit; (b) glass "sandwich" formation; (c) UV polymerization; 
(d) particle and glass removal by hydrofluoric acid; (e) porous film containing target molecule; (f) removal of 2BE molecules by thermal treatment in vacuum

to obtain MIP

Figure 6.2 SEM images of: silica particles (a); porous film surface (b); inner structure (c)

Figure 6.3 DSC thermograms of NIP hydrogel films. 115

Figure 6.4 Swelling ratio $(\% \mathrm{SR})$ variations of PAA films with $\mathrm{pH}$ 116

Figure 6.5 Recognition capacities of NIP and MIP at neutral $\mathrm{pH}$.

Figure 6.6 Reflectance spectra at $\theta=30^{\circ}$ of a: MIP1 (molar ratio of template/monomer $=1: 15), \mathrm{b}:$ MIP2 $($ molar ratio of template/monomer $=1: 39)$, c: MIP3 (molar ratio of template/monomer $=1: 387$ ), and d: NIP porous film. 120

Figure 6.7 Wavelength shifts of MIP1, MIP2, MIP3 and NIP. 120

Figure 6.8 Bragg's peak wavelength response time of MIP sensors for 2BE with initial concentration of $10 \mathrm{ppm}$. 120

Figure 6.9 Reusability test of MIP sensor $(2 \mathrm{BE}$ concentration $=100 \mathrm{ppb})$ 121 


\section{List of Tables}

Table 1.1 Organic chemicals identified in hydraulic fracturing produced water, their concentration (Lin and Nagel, unpublished data), their role in the operation

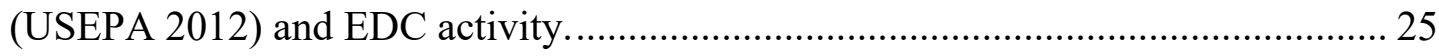

Table 5.1 Detection of DNT in real water samples..................................................... 103 


\begin{abstract}
Molecularly imprinted polymers are specialty polymers with ability of selectively capturing target molecules. They show great potential to be environmental sensors for the detection of specific contaminant. The overall research objective is to investigate the sensing ability of MIPs based on two mechanisms fluorescence quenching and reflectance for two example contaminants 2,4-dinitrotoluene and 2-butoxylethanol, which are fingerprinting contaminant of explosive manufacturing and hydraulic fracking. The water chemistry effects are explored on MIPs for their potential use as in-situ sensors in complex aquatic environments.

Fluorescent carbon dots with different surface functionality were fabricated and their environmental fate was explored. Amino-functionalized carbon dots (AC-dots) were applied to fluorescently label a molecularly imprinted polymer (MIP) for 2,4dinitrotoluene (DNT) as a template. DNT is specifically captured by the cavities in the MIP and interact with AC-dots on the surface, resulting in quenching of the fluorescence of the AC-dots. Response to DNT reaches equilibrium within $\sim 30 \mathrm{~min}$. The method has a dynamic range that extends from 1 to $15 \mathrm{ppm}$, and allows for quantitation of DNT in aqueous solutions, with a detection limit of $0.28 \mathrm{ppm}$. Selectivity tests conducted in presence of DNT analogs demonstrated the specific recognition of DNT.

The effect of sample water chemistry on carbon dots labeled molecularly imprinted polymer (AC-MIP) sensor the detection of 2,4-dinitrotoluene (DNT) was investigated. With the increase of ionic strength from $1 \mathrm{mM}$ to $100 \mathrm{mM}$, the quenching amount of MIPs decreased about $19 \%$ and $30 \%$ with $\mathrm{NaCl}$ and $\mathrm{CaCl}_{2}$ respectively. In the range of $\mathrm{pH}$ from 4 to 9 , quenching effect is slightly higher at basic environment for both MIPs
\end{abstract}


and non-imprinted polymers (NIPs) resulting from swelling properties of the films. NOM added the quenching amount to the sensor with a modified equation developed with NOM as a variable. In both lake water and tap water, DNT concentrations read by the sensors were very close to the HPLC measured DNT concentrations with the range from $72 \%$ to $105 \%$.

Molecularly imprinted polymers (MIPs) sensors for detection of 2-butoxyethanol (2BE), a pollutant associated with hydraulic fracturing contamination, were developed based on the combination of a colloidal crystal templating method and a molecular imprinting technique. MIPs exhibited higher binding than non-imprinted films (NIPs) due to the specific adsorption provided by molecular imprinting with imprinting efficiencies around 2. Optical tests were performed because of the uniformly ordered porous structure. The reflectance spectra of the sensors showed Bragg's peaks, which responded to the presence of $2 \mathrm{BE}$; peaks presented increasing red shifts up to $50 \mathrm{~nm}$ with $2 \mathrm{BE}$ concentrations in the range of $1 \mathrm{ppb}$ to $100 \mathrm{ppm}$, which allowed quantitative estimates of present $2 \mathrm{BE}$ concentration in aqueous solutions. The material has the potential for early detection of hydraulic fracturing sites contamination. 


\section{Chapter 1. Background and Introduction}

Biomolecular recognition is a process which biomolecules recognize and binds to their molecular targets such as antibodies, hormone and enzymes (Katz and Willner 2004). The interaction between biomolecule and target molecules are noncovalent bonding such as hydrogen bonding, metal coordination, hydrophobic forces, van der Waals forces, $\pi-\pi$ interactions, halogen bonding, electrostatic and or electromagnetic effects. Because these biomolecules are unstable, scientists find methods to design synthetic materials with given receptors, which are easier to prepare, cheaper and more stable (Haupt 2003). Molecularly imprinting is a useful technique mimicking the natural recognition entities for creating recognition sites in materials for target molecule (Kriz et al. 1997).

Molecularly imprinted polymers (MIPs) are crosslinked polymeric materials with molecular recognition abilities against a target molecule (template). The template is removed from the polymer matrix after polymerization and with specific binding sites left

(Figure 0.1) (García-Calzón and Díaz-García 2007).

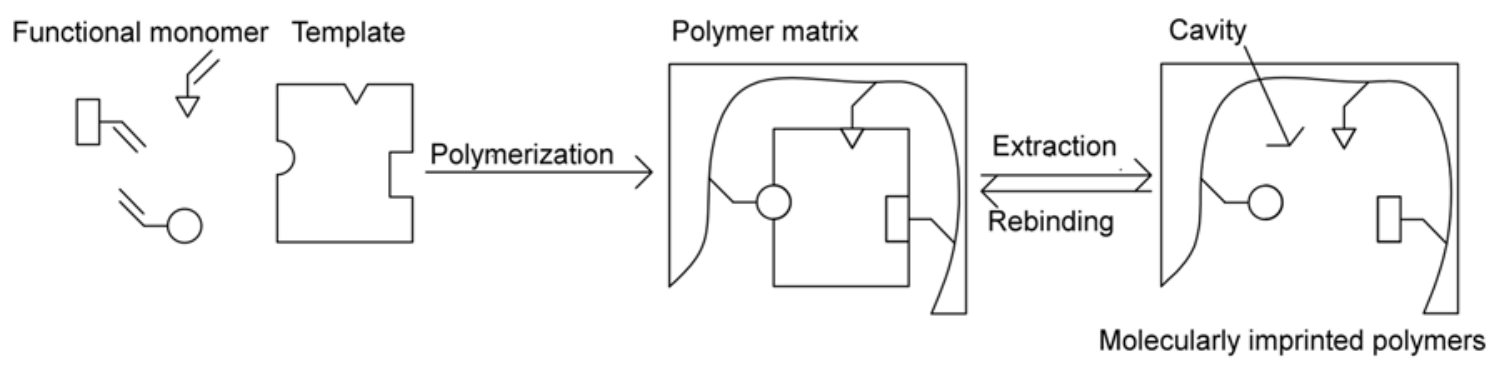

Figure 0.1 A polymer matrix is formed through polymerization by functional monomers and template. Molecularly imprinted polymers are obtained by removing templates with cavities left.

Due to their specific recognition ability, MIPs can be used for separation, purification, chemical sensors, catalysis and drug delivery. MIPs have been applied as a pretreatment 
media or solid-phase extraction (SPE) sorbent because their selective preconcentration and specific recognition of environmental pollutants followed by liquid chromatography (Hu et al. 2007, Sambe et al. 2007, Watabe et al. 2005, Xu et al. 2014). MIPs have been synthesized for the concentration and detection of a variety of pollutants present at very low levels (ppbs and below); some examples are triazines (Xu et al. 2014), TBZ (Hu et al. 2007), fluoroquinolone antibiotics (Tan et al. 2013), and triclosan (Gao et al. 2010). Compared to the widely used hydrophilic-lipophilic balanced (HLB) polymers, MIPs are more resistant to matrix effects, a common difficulty encountered in relatively dirty samples like surface water or wastewater. As a result of the better selectivity, MIPs show significantly superior performance than conventional concentration methods (Demeestere et al. 2010). This result highlights the potential for MIPs to be used in passive sampling devices (Bao and Zeng 2014, Long et al. 2014, Lydy et al. 2014).

MIPs were applied in the fabrication of sensors with capabilities to respond to a variety of physical or chemical stimulus; electrochemical, photoluminescent, and UV-vis spectroscoscopic sensors have been reported (Valero-Navarro et al. 2009, Yang et al. 2012a). In this sense, MIPs can be engineered not only to very specifically capture and concentrate organic contaminants in water at extremely low concentration, i.e. below current gold standard analytical techniques, but can also be designed to quantify the capture level and produce a signal proportional to the captured contaminant mass that can be transmitted in real time from remote sites to a central monitoring facility.

There are several methods to add photoluminescent properties to MIPs. For example, quantum dots have been combined with MIPs for fluorescence labeling but their use in environmental applications has been questioned due to their heavy metal content (Li et al. 
2010c, Zhang et al. 2011c). Carbon dots are fluorescent nanoparticles that offer a promising alternative to quantum dots for sensing, due to their low cost, benign fabrication process and negligible environmental impact (Li and Kaner 2008, Rao et al. 2009). Early studies have shown C-dots are biocompatible and less cytotoxic than Cdbased quantum dots and may even inhibit cancer cells (Hsu et al. 2013, Qu et al. 2012, Song et al. 2013). These fluorescent nanoparticles have been successfully utilized in biomedicine, optoelectronics, catalysis, and chemical sensors (Wang and $\mathrm{Hu}$ 2014a). Their application in the environmental field is particularly promising. We will begin the introduction with the fluorescence nanoparticles carbon dots and how to apply them into MIPs.

\subsection{Carbon Dots}

C-dots constitute a class of quasi-spherical, photoluminescence carbon nanoparticles with sizes below $10 \mathrm{~nm}$ (Baker and Baker 2010c). Compared to traditional semiconductor Qdots and organic dyes, photoluminescent $\mathrm{C}$-dots are superior in terms of high aqueous solubility, robust chemical inertness, easy functionalization, high resistance to photobleaching, low toxicity and good biocompatibility (Baker and Baker 2010b). As a result, much attention has also been paid to their potential application in biological labeling, bioimaging, and drug delivery (Wang et al. 2011c). During the past few years, much progress has been achieved in the synthesis, properties and application of C-dots (Shen et al. 2012a, Wang and Hu 2014b). Several low cost and environmentally benign chemical approaches have been developed for the preparation of carbon dots (Hsu et al. 2012, Wang et al. 2011a). For their strong and tunable photoluminescence, C-dots have 
been successfully applied in sensing, energy conversion and catalysis (Li et al. 2015).

As interest for both fundamental research and applications has surged dramatically in the last few years, it is inevitable CDs will find their way to natural water sources where, unlike the more well studied fullerenes and carbon nanotubes, their environmental impact and fate is mostly unknown. The presumed negligible environmental impact of carbon dots can only be confirmed if their behavior in natural systems is fully understood. However, the colloidal stability of CDs in environmentally relevant aqueous suspensions has not been investigated, and neither the effect of those conditions on their PL properties. Due to their small size and large surface area, adsorption processes are expected to play a key role in determining the physicochemical properties of C-dots (Chowdhury et al. 2013). Adsorption may alter the surface characteristics of the nanoparticles so that they become dispersed and thus mobile in aqueous media (Chowdhury et al. 2013, Lanphere et al. 2013). Natural organic matter (e.g. humic acid) and exopolymeric substances (e.g. proteins and polysaccharides) have been shown to adsorb onto carbon nanotubes and act as stabilization agents (Saleh et al. 2010); these macromolecules can potentially interact with C-dots surfaces by $\pi-\pi$ bonding and hydrophobic effects, due to the presence of $\mathrm{C}=\mathrm{C}$ double bonds as well as aromatic rings (Dimiev et al. 2012).

CDs are well known for their colloidal stability in water suspensions at low ionic strength; their PL is stable under those conditions but generally expected to decline if aggregation occurs. Other matrix chemistry effects have been reported. For example, their florescence intensities were shown to be pH-dependent, decreasing with increasing pH (Jelinek 2016). Furthermore, a study showed no significant change in fluorescence quenching of carbon dots by HA up to a concentration of $10 \mathrm{ppm}$ (Cayuela et al. 2015), while another study 
reported that the quenched fluorescence intensity of surface functionalized QDs was linearly proportional to the concentration of $\mathrm{Hg}^{2+}$ under optimum conditions (VázquezGonzález and Carrillo-Carrion 2014).

In summary, there is extensive literature on inorganic nanoparticle stability in natural waters, as well as for some common carbon-based particles such as carbon nanotubes, fullerenes and a few studies on graphene oxides, but there are no reports, to the authors' knowledge on the aggregation and surface properties of carbon dots under environmental conditions. In view of the increasing amount of research devoted to carbon dots applications for sensing and imaging, an understanding of the colloidal stability is needed as a first step towards the prediction of environmental fate, transport and potential toxicity of these nanomaterials. It has been recognized that our knowledge on nanoparticle environmental behavior needs to be continuously revised and extended to account for new materials or novel commercial applications as they emerge in order to accurately assess the associated risks (Troester et al. 2016).

\subsubsection{Functionalized Carbon Dots}

Carbon dots (CDs) as a new series of fluorescent nanomaterials have drawn great attention in recent years owning to their unique properties. Among them, functionalized C-dots have been synthesized through one-step hydrothermal carbonization of chitosan or citric acid at mild temperature (Dong et al. 2012b, Yang et al. 2012b). Some research shows surface functional groups, such as $\mathrm{C}=\mathrm{O}$ and $\mathrm{C}=\mathrm{N}$, can efficiently introduce new energy levels for electron transitions and result in the continuously adjustable full-color emissions (Nie et al. 2014). Also surface modification would increase PL quantum yield 
(QY) significantly (Zhu et al. 2013).

Typically, surface functionalization of C-dots provides an effective bridge to connect them to other systems (Ding et al. 2014). One of the simplest and most popular conjugation methods is covalent bond formation through reactive functional groups such as primary amines (Dong et al. 2012a), carboxylic acids (Liu et al. 2012a), and hydroxyls (Yu et al. 2013). Besides covalent bonding to C-Dots, metal ion coordination was employed to bind europium $\left(\mathrm{Eu}^{3+}\right)$ onto the surface of C-Dots due to the affinity between $\mathrm{Eu}^{3+}$ and oxygen-donor atoms, which subsequently quenches the fluorescence of C-Dots (Yu et al. 2013). The $\pi-\pi$ stacking interactions between C-Dots and DNA or aptamer can also be used as binding strategy for detection of nucleic acid (Li et al. 2011). Furthermore, primary alkyl-ammonium binding is used to modify 18 -crown-6-ether $(18 \mathrm{C} 6 \mathrm{E})$ on the surface of aminated C-Dots, which conjugated on reduced graphene oxide (rGO) at the same time (Wei et al. 2012). Sol gel technique is also a promising approach for engineering the surface of C-Dots with functional molecules. For example, C-Dots@MIP (molecularly imprinted polymer) was synthesized by one-pot room-temperature sol gel polymerization and applied as a fluorescence sensor for the detection of dopamine in aqueous solution (Mao et al. 2012).

It draws increasing interest to combine functionalized C-dots and MIPs for the fluorescent sensing potential. MIPs as sensors combining functionalized C-dots have been reported for recognition of Bisphenol A, 4-nitrophenol, glucose, dopamine and nifedipine (Hao et al. 2016, Jalili and Amjadi 2015, Liu et al. 2016, Wang et al. 2015b). 


\subsubsection{Fluorescence properties and Applications}

The photoluminescence (PL) properties of carbon dots vary sensitively with size, and emissions are red-shifted with increasing size (Wang and Hu 2014a). At present, the PL mechanism of carbon dots (CDs) is still under debate (Zhu et al. 2015), although different researchers have attributed their emission properties to quantum confinement effect (Li et al. 2010b), surface states determined by hybridization of the carbon backbone and the connected chemical groups (Fang et al. 2011, Hu et al. 2009), fluorophores with different degrees of p-conjugation (Bourlinos et al. 2011, Mao et al. 2010a, Srivastava and Gajbhiye 2011), and the recombination of electron-hole pairs localized within small sp2 carbon clusters embedded within a sp3 matrix. Furthermore, the PL can be tuned via surface modification or via electron/energy transfer, and numerous capping strategies have been reported (Wang and $\mathrm{Hu} 2014 \mathrm{a}$ ).

Fluorescent CDs have emerged as highly promising sensors due to its biocompatibility, simple and low cost manufacturing. CDs can be used as fluorescent probes for the detection of many cations, as well as number of anions ((Dhenadhayalan and Lin 2015)). They have been also used to detect a range of drugs, small molecules and a wide range of biomolecules (Baptista et al. 2015). These have been detected in various ways, including

fluorescence quenching (Shan et al. 2014), fluorescence 'turn-off turn on' methods (Zheng et al. 2013), modified electrodes (Wang et al. 2012b), and target recognition (Wang et al. 2013b).

\subsection{Molecularly Imprinted Polymers}

There are two main methods to create molecularly imprinted polymers. The first one is 
called the self-assembly approach, which involves only noncovalent interactions during the process all elements of the MIP combine and interact with the template molecule bound. The second is preorganized approach in which the template molecule covalently links to the monomer while, after polymerization, the monomer is cleaved from the template molecule (Kriz et al. 1997). A hybrid of these two called semi-covalent approach. There are covalent bonds between the template and the functional monomers before polymerization, while after removing the template, the re-binding of the target molecule to the MIP occurs by non-covalent interactions (Yan 2004).

Several elements including a template, functional monomers, a cross-linker, an initiator are involved in molecular imprinting process. Pyrogenic solvents and extraction solvents may also be used. The template and functional monomers with the specific functional groups participate in binding interactions and create the imprinted binding sites. The cross-linker provides a rigid polymer matrix and it holds the functional monomers to stabilize the imprinted binding site and contribute in determining the nature of MIPtemplate selectivity. Initiators initiate free radical polymerizations in the presence of templates. The pyrogenic solvent serves to bring all the components in the polymerization, while the extraction solvent removes the template to create a cavity in the polymer matrix (Cormack and Elorza 2004).

The chemical equilibrium in the polymerization solution is described by the following equation:

$[\mathrm{MTM}]=\mathrm{K}^{2}[\mathrm{~T}][\mathrm{M}]^{2}$ Equation 0.1 where $\mathrm{K}$ is the association constant; $[\mathrm{T}]$ is the concentration of template molecule; $[\mathrm{M}]$ is the concentration of functional monomer; and $[\mathrm{MTM}]$ is the concentration of the desired 
template monomer complex.

If we assume the association between two functional monomers and one template are independent and identical, we can get the following equation,

$\mathrm{N}=\mathrm{N}_{\mathrm{S}}+\mathrm{N}_{\mathrm{NS}}=\mathrm{P}\left(\mathrm{K}^{2} \cdot[\mathrm{L}] \cdot[\mathrm{MTM}]+\mathrm{K} \cdot[\mathrm{L}] \cdot\left(\left[\mathrm{M}_{0}\right]-2[\mathrm{MTM}]\right)\right) \quad$ Equation 0.2

where N, NS, and NNS are total, specific, and nonspecifically binding, respectively, L is the free ligand concentration, and $\mathrm{P}$ is a polymer concentration factor (in relation to the concentrations of the template molecules and the polymer).

The selectivity of MIPs can be calculated when other values are obtained from experiments.

By applying thermodynamic theory, the trend of molecular imprinting reactions can be characterized with the following equation,

$\Delta \mathrm{G}=\Delta \mathrm{G}_{\text {(trans+rot) }}+\Delta \mathrm{G}_{\text {(rotors) }}+\Delta \mathrm{G}_{\mathrm{H}}+\sum \Delta \mathrm{G}_{\mathrm{i}}+\Delta \mathrm{H}_{\text {conform }}+\Delta \mathrm{G}_{\mathrm{vdw}} \quad$ Equation 0.3

where $\Delta G$ is the change in Gibbs free energy for the formation of the monomer-template complex; $\Delta \mathrm{G}_{\text {(trans+rot) }}$ is the change in Gibbs free energy for both translational and rotational; $\Delta \mathrm{G}_{(\text {rotors })}$ is the change in Gibbs free energy caused by restricted rotational motion template molecules; $\Delta \mathrm{G}_{\mathrm{H}}$ is the change in Gibbs free energy of the whole groups participated in the reactions; $\Delta \mathrm{H}_{\text {conform }}$ is the strain energy in the complex in relation with the introduction of unfavorable bond lengths, bond angles, dihedral angles, and so on; and $+\Delta \mathrm{G}_{\mathrm{vdw}}$ is the change in Gibbs free energy by van der Waals forces.

In some cases, some changes in Gibbs free energy are negligible; the formula can be simplified as:

$\Delta \mathrm{G}=\Delta \mathrm{G}_{\text {(trans+rot) }}+\Delta \mathrm{G}_{\text {(rotors) }}+\sum \Delta \mathrm{G}_{\mathrm{i}}+\Delta \mathrm{G}_{\mathrm{vdw}} \quad$ Equation 0.4 Template and functional monomers can form multiple binding sites. Molecular 
recognition characteristics of MIPs are attributed to pre-organization of functional groups and size and shape specificity of binding. The selectivity of MIPs is highly dependent on the number and intensity of interactions between template molecules and imprinted groups, in addition to the shape and rigidity of the template molecules.

According to molecular cluster theory, the shape of nanocavities is complementary to the template used like lock-key. This lock-key principle is constituted by many binding sites, with continuous distribution of affinities of template. The template-template interaction should be paid with more attention for the cavities in the MIPs with the formed molecular clusters of chiral imprinted molecules. MIPs prepared with crystal nuclei have larger adsorption ability (Cao et al. 2012).

\subsubsection{MIPs and their applications as environmental sensors}

Polymer-based sensors attract attention due to the simplicity and low cost of manufacturing, improved shelf life and possibilities for mass production with potential of minimizing batch-to-batch variation in performance. The lock-key features of MIPs attracted increasing attention, and MIPs have been considered as promising alternatives to their biological counterparts to develop novel matrices in diverse research fields. An interdisciplinary research area combining sensors and MIPs has spontaneously emerged. To evaluate the effectiveness of sensors, the key features include the recognition selectivity and specificity, limits of detection and quantification, and overall quality and robustness of the results (Uzun and Turner 2016).

Satisfactory MIPs-based sensors contain two parts: recognition sites and a signal transducer (Xu et al. 2012). The recognition process plays an important role in their sensing performance. The recognition process is controlled by three major factors: the 
quantity of the functional groups participating in the interaction, their correct arrangement within the cavity, and the shape of the cavity itself (Piletsky and Turner 2008). The specificity can be provided solely by the shape and size of the cavity although the specificity is enhanced when the template interacts with one or more properly oriented functional monomers (Vasapollo et al. 2011).

One recognition mechanism observed in MIP systems is the conformational change in the polymer induced by template interaction. Stimuli such as $\mathrm{pH}$ or the presence of template can translate into the swelling/shrinking behavior when polymer matrix present as hydrogels (Vasapollo et al. 2011). Photonic polymer sensor and pH-responsive MIPs have been developed based on the conformational change effect (Kanekiyo et al. 2008, Wang et al. 2012a).

Optical devices based on the fluorescent properties of template can be achieved through three kinds of design of MIP-based sensors including affinity sensors, receptor sensors and catalytic sensors. The detection principle of the affinity MIP sensor, immunosensortype device is based on the measurement of the concentration of the template adsorbed by MIP immobilized on the detector surface (Johnson-White et al. 2007). However, not all analytes can be easily modified with dyes and the modification itself can change the affinity of the analyte. For MIP-based receptor sensors, they most often measure the change in membrane electroconductivity, induced by specific interaction of MIPs with template molecules (Sergeyeva et al. 1999). The catalytic sensors combine MIPs possessing catalytic properties with traditional electrochemical or optical transducers (Lakshmi et al. 2009). 


\subsubsection{Characterization methods of MIPs}

Batch rebinding is a method to evaluate binding capacity and selectivity for MIPs. The procedure is demonstrated in Figure 0.2. It is assumed that after serial incubation equilibrium between the template and the imprinted polymer is reached.

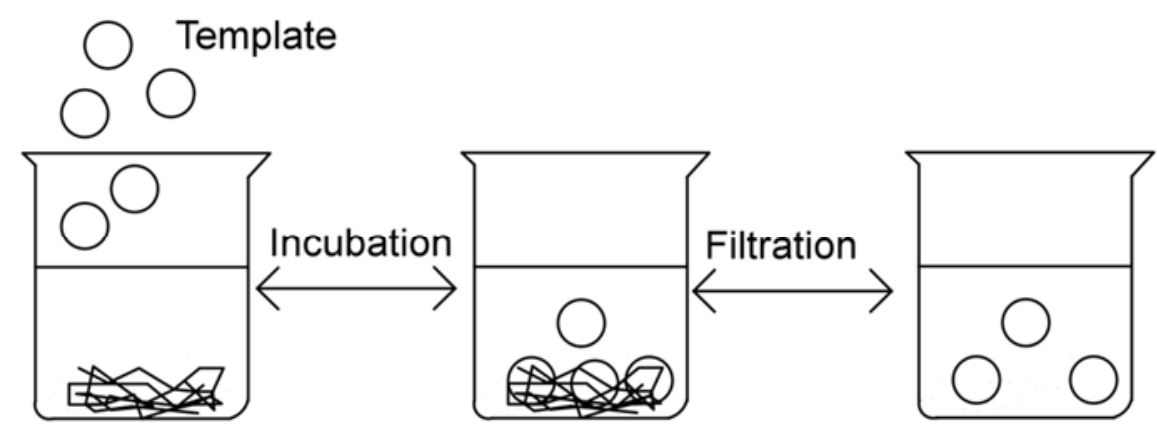

Molecularly imprinted polymer Bound template Free template

Figure 0.2 Batch rebinding approach is accomplished by serial incubation and then the polymer is filtrated to determine the free template in each vessel (García-Calzón and Díaz-García 2007).

The reversible reaction is:

$R_{\text {free }}+L_{\text {free }} \underset{k_{-1}}{\stackrel{k_{1}}{\rightleftarrows}} R L$

Equation 0.5

where $\mathrm{k}_{1}$ stands for adsorption constant, and k-1 stands for the desorption constants. At equilibrium:

$\frac{\left[L_{\text {free }}\right]\left[R_{\text {free }}\right]}{[R L]}=\frac{C\left(N_{t}-B\right)}{B}=\frac{k_{-1}}{k_{1}}=k_{d}$ Equation 0.6

where $\left[R_{\text {free }}\right]=N_{t}-B, N_{t}$ is the amount of existing active centers per unit of volume in the MIP, and $k_{d}$ stands for the equilibrium desorption constant. The equation can be rearranged to:

$\frac{B}{N_{t}}=\frac{C}{k_{d}+C}$

Equation 0.7 
The amount of bound template per gram of imprinted polymer can be calculated. (GarcíaCalzón and Díaz-García 2007).

Chromatography can separate the components and measure the relative proportions of analytes in a mixture. One of the main applications of MIPs is as chromatographic stationary phases for selective preconcentration of target component. Polymer particles are manually packed into refillable columns and connected to chromatography instrument. Theoretical plate numbers $\left(\mathrm{N}_{\mathrm{sys}}\right)$ can be calculated for each analyte using classical chromatographic theory, assuming skewed peaks, according to the following equation:

$$
\begin{array}{ll}
N_{\text {sys }}=\frac{41.7\left(t_{R} / W_{0.1}\right)^{2}}{B / A+1.25} & \text { Equation } 0.8
\end{array}
$$

where $t_{R}$ is the retention time, $\mathrm{W}_{0.1}$ is the peak width at the corresponding peak height fraction, and $\mathrm{B} / \mathrm{A}$ is the asymmetry factor.

It is also a quick and easy method for analyzing binding properties of MIPs. When the template binds to the MIPs in the column, the polymer becomes saturated and the amount of template eluting from the column gradually increases, providing an obvious increase in the characteristic peak (Barahona et al. 2010).

UV-vis spectroscopy and ${ }^{1} \mathrm{H}$ NMR are two common methods in determining the intermolecular interaction between functional monomers and template molecule (Shi et al. 2007).

Ultraviolet-visible spectroscopy uses light in the visible and adjacent (near UV and nearinfrared) ranges. It can be an adsorption spectroscopy or reflectance spectroscopy and often used in analytical chemistry for the quantitation of different substances. In a UV spectroscopic titrations experiment, template solution titrates in functional monomers 
solutions of different concentrations. After equilibrating for a while, the changes of absorbance $(\triangle \mathrm{A})$ of these solutions are measured at a specific wavelength by a UV spectrophotometer (Chen et al. 2011). From the results of the experiment, we can calculate the dissociation constants for the polymerization interaction, as well as the amount of unreacted template (Karim et al. 2005).

Nuclear magnetic resonance (NMR) spectroscopy is a research technique to determine the physical and chemical properties including structure, dynamics, reaction state, and chemical environment of atoms or the molecules. NMR analysis is used to identify substance with good resolution. Different functional groups are distinguishable with obvious signals. However, NMR spectroscopy is relatively expensive and has a very large liquid helium-cooled super-conducting magnet. NMR active nuclei such as ${ }^{1} \mathrm{H}$ and ${ }^{13} \mathrm{C}$ absorb electromagnetic radiation in a magnetic field.

Gibbs free energy change can reflect the extent of non-covalent monomer-template interactions. NMR spectroscopy has revealed the mechanisms of molecular recognition of MIPs and predicts binding capacities by calculating monomer-template dissociation constants (Sellergren et al. 1988, Whitcombe et al. 1998).

Fourier-Transform Infrared Spectroscopy (FT-IR) provides an infrared spectrum to identify chemical bonds in a molecule for samples. It can produce a profile of functional groups and covalent bonding information of the polymer. Usually there are $\mathrm{O}-\mathrm{H}$ and $\mathrm{C}=\mathrm{O}$ stretching vibration in the MIPs with empty cavities, so that the target-containing MIPs have peaks shifted to lower frequencies (Javanbakht and Akbari-Adergani 2012).

The structure of MIPs is very important on their imprinting performance. More binding sites are available with a larger surface area. Additionally, polymers have different 
morphologies as shown in Figure 0.3.

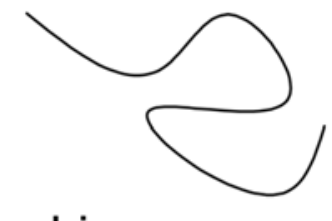

Linear

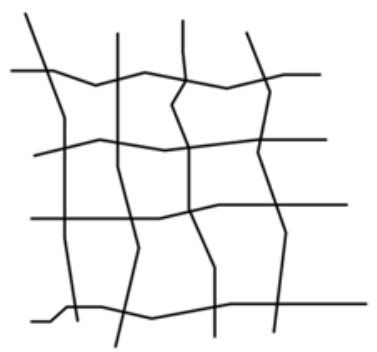

Macroscopic Network

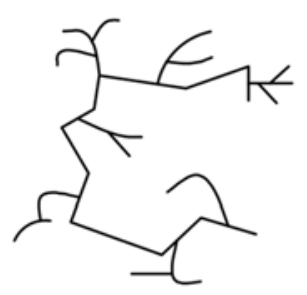

Branched

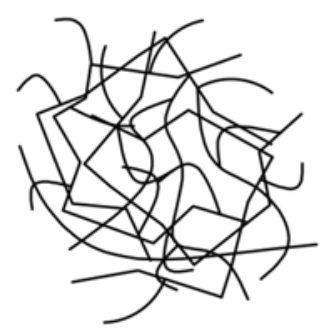

Microgel

Figure 0.3 Different topologies of polymers can be linear, branched, macroscopic network and microgel, which is important in imprinting effects of MIPs (Cormack and Elorza 2004).

Morphological information of MIPs can be achieved by microscopy techniques like scanning electron microscope. BET analysis provides precise specific surface area evaluation of materials for studying porosity and particle size.

A scanning electron microscope (SEM) uses electrons instead of light to produce images. The electrons interact with atoms in the sample, producing various signals including secondary electrons, back-scattered electrons, X-rays, etc. These signals can be detected and give information of the samples' surface structure and chemical composition. Because most of MIPs are nonconductive, a thin layer of conductive material like gold, platinum or carbon needs to be coat on them using a sputter coater. SEM can often be used to image macropores of MIPs. 
Braunauer-Emmett-Teller (BET) theory explains the physical adsorption of gas molecules on a solid surface and is the underlying theory for the measurement of the specific surface area of a material. Nitrogen sorption porosimetry involves a fixed mass of dry polymer being exposed to nitrogen at a series of fixed pressures. Sorption isotherms can be constructed by the amount of gas sorbed as a function of pressure. The specific surface area $\left(\mathrm{m}^{2} / \mathrm{g}\right)$, specific pore volume $(\mathrm{ml} / \mathrm{g})$, average pore diameter and pore size distribution can be calculated based on BET and mathematical models (Cormack and Elorza 2004).

Surfaces of MIPs with recognition abilities can be divided into two general categories: homogeneous and heterogeneous shown in Figure 0.4. If the binding cavities have identical shapes and therefore the binding sites have the same affinity and selectivity, it can be considered as a homogeneous material. If the binding cavities have different shapes so as the binding sites have varying affinity and selectivity, then it should be a heterogeneous material (Umpleby et al. 2004).

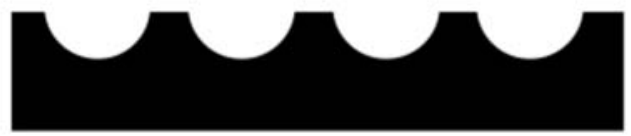

Homogeneous

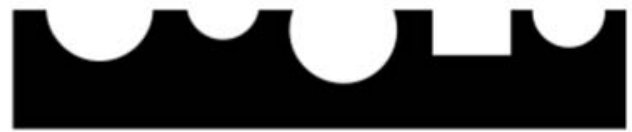

Heterogeneous

Figure 0.4 Schematic illustration of binding site homogeneity and heterogeneity in MIPs (Umpleby et al. 2004)

\subsubsection{Stober method}

MIPs can be fabricated by different polymerization methods. Bulk polymerization has 
been used extensively, followed by grinding and sieving to obtain the MIP particles. Although simple, this approach is usually unsatisfactory due to low yields, and irregular size and shape of the particles that complicate further uses (He et al. 2007). Micrometer and nanometer sized MIP particles constitute an improvement from the bulk polymerization technique, but the separation of the MIP beads from the sample specially in natural environments still remains a challenge (Haupt 2012). Recently, researchers have developed an original procedure that combines molecular imprinting and colloidal crystal to prepare polymers with 3D, highly ordered, macroporous structures and specific binding nanocavities for a rapid assay to detect organic compounds like bisphenol A (Griffete et al. 2011a, Griffete et al. 2011b), atrazine (Wu et al. 2008a) and specific stimulants like theophylline or ephedrine (Hu et al. 2008a). The high sensitivity and specificity observed in these polymeric systems is mainly due to the high surface-tovolume ratios of the structure that allow for a more efficient mass transport in submicrometer-sized pores and enhance surface reactions. In particular, highly ordered porous materials based on hydrogels are able to swell or shrink in aqueous solution upon molecular recognition or environmental conditions leading to a change in optical properties (Hu et al. 2006, Hu et al. 2008b). The highly controlled pore structure achieved through the colloidal template technique is especially beneficial in sensor applications, since it gives elevated specific surface areas, more interaction sites, efficient mass transport, easier accessibility to the active sites through the interconnected macroporous system, as well as high specificity to analytes of the nanocavities (Holtz and Asher 1999, Sharma et al. 2004). 


\subsection{DNT and its Environmental Sensors}

\subsubsection{DNT and its retarding effects on vinyl polymerization}

The widespread application of DNT in the manufacturing of a diverse set of chemicals has resulted in substantial contamination of soil and water (Xu and Jing 2012). DNT is a precursor and an inevitable byproduct of the manufacturing process of 2,4,6trinitrotoluene (TNT), an explosive used worldwide. DNT is also involved in the production of polyurethane foam products, dyes, plastics, plasticizers, deterrent coatings, and burn rate modifiers for propellants (Lent et al. 2012). DNT is commonly found in surface water, groundwater and soils near the former ammunition factories, facilities that manufacture or process DNT (Reddy et al. 2014). The moderately soluble DNT has low volatility and demonstrated toxicity to the hematopoietic, cardiovascular, nervous, and reproductive systems of humans (Lent et al. 2012, Tchounwou et al. 2003). Therefore, the detection of DNT is of utmost importance, and a significant amount of effort has been devoted to the development of suitable sensors, both in gas and liquid phase (Bosco et al. 2012, Kabessa et al. 2016, Toal and Trogler 2006, Wang et al. 2016). Optical detection techniques, colorimetric or fluorimetric based, have the advantage that they can be portable and used in-situ. Fluorescence sensors are especially suited for detection of nitroaromatic compounds such as DNT, due to their ability to quench emission of excited species (Goodpaster and McGuffin 2001a).

DNT may work as a retarder in vinyl polymerization because aromatic nitro compounds act as inhibitors and show greater tendency toward more reactive and electron-rich radicals. Nitro compounds have very little effect on methyl acrylate and methyl methacrylate but inhibit vinyl acetate and retard styrene polymerization. The 
effectiveness increases with the number of nitro groups in the ring. The mechanism of radical termination involves attack on both the aromatic ring and the nitro group. The reactions are shown in Figure 0.5 and Figure 0.6.

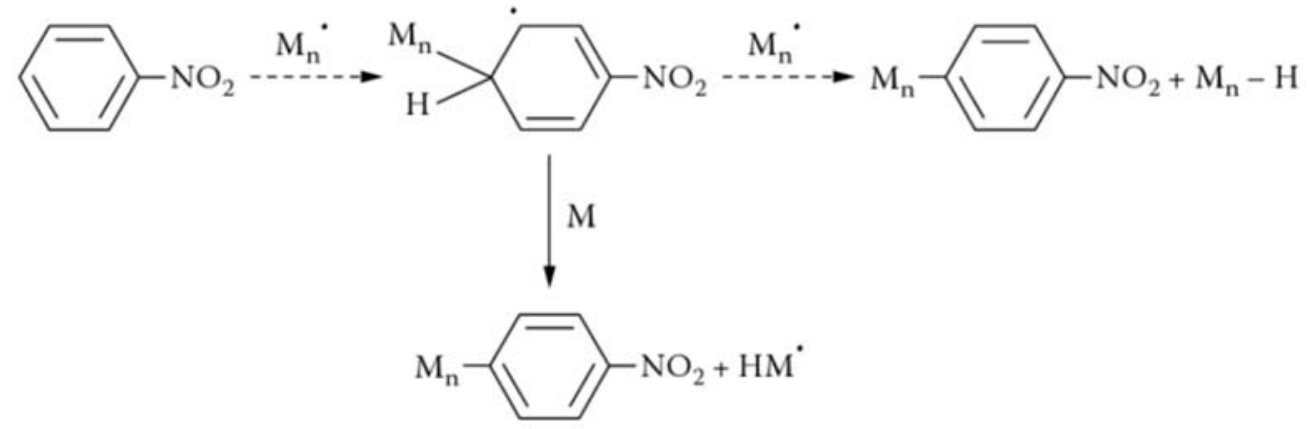

Figure 0.5 Radicals attack on the ring of nitroaromatic compounds

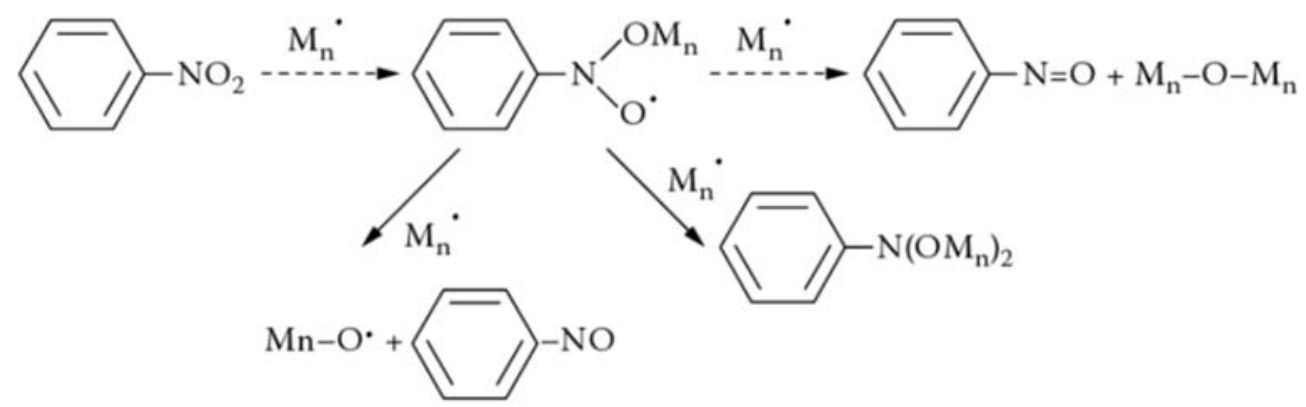

Figure 0.6 Radicals attack on the nitro groups of nitroaromatic compounds

\subsubsection{DNT sensors}

Fluorescence based detectors for DNT have been reported using fluorescent conjugated polymers, hybrids composed of inorganic materials functionalized with an organic fluorophore, and quantum dots (Peveler et al. 2016, Salinas et al. 2012). The latter has shown advantages over the previous approaches, as these nanoparticles do not suffer from instability with respect to temperature, $\mathrm{pH}$, and photobleaching that characterizes the organic-based detection methods (Stringer et al. 2010). Although the exact chemical 
composition of Q-dots varies, they are typically made of cadmium selenide or sulfide (Zhang et al. 2011a), which have raised questions regarding their toxicity. Even though fluorimetric based sensing techniques are very sensitive, they lack specificity. One strategy to increase the specificity of the sensing devices is the capture of the target molecules by Molecularly Imprinted Polymers (MIPs) (McCluskey et al. 2007a). MIPs are robust and can be designed to be insoluble in the media of interest (Wulff 2001). Fluorescent sensors based on Q-dots labeled MIPs have been developed for nitroaromatic explosives like DNT and TNT in aqueous phase (Xu et al. 2013), but the loss of nanoparticles or leaching of toxic heavy metals may result in serious environmental pollution so that the use of CdTe quantum dots is not suitable for an aquatic environment.

\subsubsection{The effects of water chemistry and interferences}

The MIP based sensors can be successfully used for quantitative detection of specific contaminants. In order to obtain a useful sensor device, different transducer mechanisms have been combined with MIPs, such as optical, chemical or electrical properties variations. Among them, the optical MIP-based molecular recognition sensors have been developed due to the covalent linkage of MIPs to optical transducers. The MIP binding

efficiency, recognition processes and selectivity have been intensively studied by optical means due to the general high sensitivity and simplicity of the utilization of optical techniques (Henry et al. 2005). The sensing properties can also be characterized by parameters such as linearity, limit of detection, sensitivity, selectivity, reproducibility, accuracy, robustness, response time and lifetime (Li et al. 2012). However, the effects of sample characteristics commonly encountered in natural waters (i.e. variations of $\mathrm{pH}$, 
ionic strength, natural organic matter content) on the sensing performance ofoptical MIPs are not fully understood. MIPs have been reported which respond to stimuli such as change in temperature (Pan et al. 2013), pH (Kanekiyo et al. 2003), and ionic strength (Wu et al. 2010). Interferences are another concern because they can affect the recognition of MIPs. In the context of this study, we define interference as a compound that will hinder the access to capture sites of the target molecule by non-specific adsorption onto the polymer surface resulting in pore blocking, steric repulsion or electrostatic interactions at or near the interface, and produce a decrease in the capacity of the MIP. Potential interferences in natural waters are dissolved organic matter, dissolved salts, inorganic colloidal particles, and microorganisms. Given the fact that most efforts have been devoted to the synthesis of MIPs as SPE media, research in this area has been directed mostly towards the conditioning, washing and eluting stages of the process rather than the capture and sensing, and much of the actual mechanisms by which this interference may occur are not fully understood. The potential for MIP-based sensors to be used in-situ is promising if the influences of these parameters are explored and interpreted.

Some research has been reported as the use of MIPs as the sorbent for solid-phase extraction of natural water samples (Davoodi et al. 2014, Kueseng et al. 2009). The direct use of MIPs and MIP-enabled devices as sensors in natural waters is not fully explored (Alizadeh et al. 2010, Hao et al. 2016), especially on the effects of different parameters in the water chemistry matrix. Conductometric transducer based atrazine sensor allows the monitoring of atrazine both in contaminated and uncontaminated natural waters as the detection limit is $1 \mathrm{ppb}$. The only draw back is that it required laboratory based 
instrumentation and is not suitable for field studies (Rao et al. 2007). Atrazine and its analogs simazine, ametryn and propazine could be successfully extracted from natural water samples, using atrazine as the template molecule (Wen et al. 2012). In another study, diphenolic Acid (DPA) and bisphenol A (BPA) were used as dummy template molecules for the capture of tetrabromobisphenol A (TBBPA). The reported recoveries of TBBPA in tap, river and lake water ranged from $85 \%$ to $97 \%$ with detection limit as low as $2 \mathrm{ng} / \mathrm{mL}$ (Yin et al. 2012)

Fluorescence based sensors are particular suited for field use, as they are generally sturdy and easy to measure. In this field, fluorescent nanoparticles are preferable to fluorescent polymers, as they have shown to be brighter and more stable; however, leakage of the nanomaterial in to the sample is a concern if the particles are not strongly bonded to the MIP matrix. Sample chemical matrix may produce additional interference by interacting or preventing interaction between the target compound and the fluorescent material, leading to over or underestimations of the true concentration of the target. Detection of contaminants in natural waters at environmentally relevant concentrations is particularly challenging, due to the complex and potential variability of its chemical composition.

\subsection{Hydraulic fracturing and 2-BE-MIPs sensors}

\subsubsection{Hydraulic fracturing and its fingerprinting contaminants}

The rapid rise in unconventional oil and natural gas (UOG) operations combining directional drilling and hydraulic fracturing (fracking) increases the opportunity for air and water pollution from these processes. UOG operations involve the injection of millions of gallons of water and thousands of gallons of more than 750 chemicals 
(Committee on Energy and Commerce 2011), in addition to naturally occurring compounds such as radioactive materials, salts, arsenic, selenium, strontium, and barium which are embedded in the shale layer (Fontenot et al. 2013, Maule et al. 2013, Orem et al. 2014, The Shale Gas Subcommittee of the Secretary of Energy Advisory Board 2011, Warner et al. 2012).

UOG operations release large amounts of reproductive, immunological and neurological toxicants, carcinogens, and endocrine disrupting chemicals (EDCs) into the environment (Colborn et al. 2010) . The effects include infertility, miscarriage, impaired fetal growth, low birth weight, preterm birth, and birth defects (Attarchi et al. 2012, Christiansen et al. 2008, Dadvand et al. 2013, Llop et al. 2010, Lupo et al. 2011, McKenzie et al. 2014). Many of these same reproductive health impacts have also been observed in companion and farm animals living in intensively drilled areas in the United States (Bamberger and Oswald 2012). We previously assessed the EDC activities of 24 fracturing chemicals on 5 hormone receptors, reporting antagonist activities for the majority of the chemicals examined (Kassotis et al. 2014), which was the first report of direct receptor activity for many of them (Chang et al. 2004, Creasy 1985, Devillers et al. 2002, Sohoni and Sumpter 1998, Thayer and Belcher 2011, Thomas and Budiantara 1995). We also found that surface and ground water from fracturing fluid spill sites in Garfield County, Colorado, exhibited higher EDC activities than samples collected outside the active drilling region (Kassotis et al. 2014).

EDCs are part of the emerging contaminants that are being found with increasing frequency in wastewater (Hecker et al. 2002, Johnson and Sumpter 2001). This concern stimulates the development of new selective analytical methods for their detection 
(Auriol et al. 2006, Matthiessen and Johnson 2006). Conventional methods to detect organic compounds involve not only expensive instrumentation, but also a large number of separating analytical procedures, resulting in a complex, time-consuming, and laborious screening procedure. Furthermore, detection in remote areas with difficult access adds complexity and inaccuracy due to sampling errors by non-trained personnel and sample conservation issues. For this reason, the development of novel approaches for easy and rapid detection of selected organic contaminants is highly desirable. Molecular imprinting is a well-established technique used to synthesize molecularly imprinted polymers (MIPs) with specific molecular recognition nanocavities (Caykara et al. 2003). Owing to the complementarity in shape and binding sites, the created nanocavities exhibit high selectivity towards the imprinted molecules, including a large and diverse set of important organic compounds including hormones and metal ions (L. Chen et al. 2011, S. Wei et al. 2006, Zhu L et al. 2003).

MIPs were applied in the fabrication of sensors with capabilities to respond to a variety of physical or chemical stimulus (Valero-Navarro et al. 2009, Yang et al. 2012a). Moreover, the high specificity allows for the fabrication of MIPs arrays targeting a definite group of contaminants. Careful selection of contaminants present in effluents from certain industrial activity and their simultaneously detection combined with their relative concentration can be regarded as a "fingerprint" of contamination from a particular source (Cecinato et al. 2014). Fingerprinting has been applied in the identification of contamination sources in oil spills (Mulabagal et al. 2013, Pei et al. 2013), gasoline leakage from underground storage tanks (Liang et al. 2012), fisheries (Stuart et al. 2014), and food industry wastewater (Wang et al. 2013a). 
Similarly, fingerprinting compounds can also be identified for hydraulic fracking. The most important contaminants are those that have been recognized as EDCs due to their health impact and confirmed presence in hydraulic fracturing waste water: more than 130 fracturing chemicals have been identified as known or potential EDCs (Colborn 2011, Committee on Energy and Commerce 2011, Kassotis et al. 2014, Kassotis et al. 2016). 2BE has been identified in hydraulic fracking wastewater in high concentrations, up to grams per liter, and therefore can be considered an indicator of contamination (Thacker et al. 2015), which is also shown in Table 0.1.

Table 0.1 Organic chemicals identified in hydraulic fracturing produced water, their concentration (Lin and Nagel, unpublished data), their role in the operation (USEPA 2012) and EDC activity.

\begin{tabular}{|c|c|c|c|c|}
\hline \multirow[b]{2}{*}{ Chemical } & \multirow[b]{2}{*}{ Application } & \multicolumn{3}{|c|}{ Type of EDC Activity^ } \\
\hline & & $\begin{array}{c}\text { Anti- } \\
\text { Estrogen }\end{array}$ & $\begin{array}{c}\text { Anti- } \\
\text { Androgen }\end{array}$ & $\begin{array}{c}\text { Anti- } \\
\text { Thyroid }\end{array}$ \\
\hline 2-Butoxyethanol & 55,000 Surfactant, corrosion inhibitor & * & * & * \\
\hline Benzene & 1,338 Paraffin inhibitor & $-20 \%$ & $-33 \%$ & $0 \%$ \\
\hline Cumene & 9 Paraffin inhibitor & $-43 \%$ & $-38 \%$ & $0 \%$ \\
\hline Ethylbenzene & 88 Paraffin inhibitor & $-14 \%$ & $-30 \%$ & $0 \%$ \\
\hline Naphthalene & 80 Surfactant, corrosion inhibitor & $-39 \%$ & $-37 \%$ & $-40 \%$ \\
\hline O/M/P-Xylene & 1920 Paraffin inhibitor & $-33 \%$ & $-13 \%$ & $0 \%$ \\
\hline Toluene & 3,755 Paraffin inhibitor & $-8 \%$ & $-10 \%$ & $0 \%$ \\
\hline Trimethylbenzene & 259 Surfactant, stabilizer & $-6 \%$ & $-6 \%$ & $-2 \%$ \\
\hline
\end{tabular}

^Antagonist activity at $100 \mu \mathrm{M}$ was measured as percent inhibition of estradiol, dihydrotestosterone or T3.

${ }^{*}$ Not yet measured

\subsubsection{UV-vis reflectance and 2BE MIP sensors}

Optical sensors based on the conformational change in presence of template are due to shrinking or swelling behaviors of MIPs. Inverse opal films of MIPs based on colloidal crystal template method have been reported.

Due to the uniformly ordered porous structure, the reflection peaks recorded by the 
spectrophotometer could be interpreted as interferences between light reflection on dense (111) plane (Griffete et al. 2012). The peak, $\lambda_{\max }$, for the porous hydrogel is related to the structure of the porous film by the Bragg equation:

$\lambda_{\max }=1.633(\mathrm{~d} / \mathrm{m})\left(\mathrm{D} / \mathrm{D}_{0}\right)\left(\mathrm{na}^{2}-\sin \theta^{2}\right)^{0.5}$

Equation 0.9

where $d$ is the sphere diameter of the silica colloidal particles used to fabricate the porous film, $m$ is the order of Bragg diffraction, $\left(D / D_{0}\right)$ is the degree of swelling of the gel $(D$ and $D_{0}$ denote the diameters of the gel in the equilibrium state at a certain condition and in the reference state, respectively), $n_{a}$ is the average refractive index of the porous gel at a certain condition, and $\theta$ is the angle of incidence.

These highly ordered structures are made from hydrogel polymers, they can swell or shrink in response to various stimuli including $\mathrm{pH}$, glucose, ionic strength, temperature and so on, leading to a change in reflection wavelength accompanied by a visually structure color change (Chen et al. 2016). These inverse opals of molecularly imprinted polymers have been used for the detection of bisphenol A (Griffete et al. 2012), valillin (Peng et al. 2012), transferrin (Wu et al. 2017), and nanoparticles (Gam-Derouich et al. 2017). $2 \mathrm{BE}$ as a fingerprinting contaminant of hydraulic fracking will be used as the template of the molecularly imprinted polymers and the binding and sensing performance of the sensors will be explored. 


\section{Chapter 2. Research Objectives}

The objective of this work is to investigate the sensing ability of MIPs and MIPs combined with functionalized CDs. The water chemistry effects are explored on CDs and CDs-labeled MIPs for their potential use as in-situ sensors in complex aquatic environments.

In Chapter Three, we study for the first time the colloidal stability of carbon dots, an emerging carbon nanomaterial, in natural water conditions (i.e. variable salinity, $\mathrm{pH}$, dissolved organic matter) and the effect of the transformations they undergo under such circumstances on their fluorescence properties. Two types of carbon dots were considered: as obtained from thermal treatment of organic molecules, and surface functionalized, to represent differences in the surface chemistry. The particles were synthesized and characterized with respect to size and surface chemistry. Aggregation was investigated under environmental relevant conditions and DLVO theory applied to the modeling of particle stability.

In Chapter Four, we fabricated highly porous molecularly imprinted poly(methyl acrylate-co-acrylic acid) (PMAAA) films to solve the disadvantages of previously reported sensors. The porosity and surface area of films were provided by the bulk polymerization of the monomer solution and the template molecule in the pore space of a silica colloidal crystal. AC-dots were covalently bonded to the polymeric film linking amino groups and carboxyl groups with N-hydroxysuccinimide (NHS) and N-(3Dimethylaminopropyl)-N'-ethylcarbodiimide hydrochloride (EDC) as catalysts. Compared with physical attachment or simple coating approaches to C-dots labeled sensors, the strong covalent bonding of the nanoparticles was expected to provide 
superior durability, avoiding the loss of nanoparticles and ensuring fluorescence stability. The easy detection was based on the amount of captured DNT molecules through fluorescence quenching.

In Chapter Five, we investigated the field application of MIP sensor labeled with fluorescent nanoparticles (carbon dots) for the detection and quantification 2,4dinitrotoluene (DNT) in natural water conditions. The effect of salinity, $\mathrm{pH}$ and dissolved organic matter on the sensor performance was analyzed. Two factors may potentially play important roles: interference with the nonspecific and specific adsorption of the DNT onto the MIP, and additional quenching by solutes other than the DNT. A methodology was established to minimize those effects and/or create an adjusted calibration for determination of DNT concentrations under different background water matrixes.

In Chapter Six, our objective is to develop the highly ordered porous photonic 2butoxyethanol-MIP film as UV-vis reflectance sensor. $2 \mathrm{BE}$ has been identified in hydraulic fracking wastewater in high concentrations, up to grams per liter, and therefore can be considered an indicator of contamination (Thacker et al. 2015). By combining MIP with inverse opals, the sensor will selectively adsorb 2BE when present in the tested solutions, resulting in a change the conformational structure of the film and consequently a shift of the peak wavelength of the light reflected by the polymer (Wu et al. 2008b). 


\section{Chapter 3. Effect of water chemistry on the aggregation and \\ photoluminescence behavior of carbon dots}

Carbon dots (CDs) constitute a class of quasi-spherical, photoluminescence carbon nanoparticles with sizes below $10 \mathrm{~nm}$ Carbon dots (CDs) constitute a class of quasispherical, photoluminescence carbon nanoparticles with sizes below $10 \mathrm{~nm}$ (Baker and Baker 2010a). Their easy fabrication and presumably negligible environmental impact make them an attractive alternative to inorganic quantum dots ( $\mathrm{Li}$ and Kaner 2008, Rao et al. 2009). Early studies have shown CDs are biocompatible and less cytotoxic than Cdbased quantum dots and may even inhibit cancer cells (Hsu et al. 2013, Qu et al. 2012, Song et al. 2013). These fluorescent nanoparticles have been successfully utilized in biomedicine, optoelectronics, catalysis, and chemical sensors (Wang and $\mathrm{Hu} 2014 \mathrm{~b}$ ). Their application in the environmental field is particularly promising. Semiconductor quantum dots (SQDs) and graphene quantum dots (GQDs) can be distinguished within the large group of carbon based nanoparticles since SQDs and GQDs are perfect spherical nanocrystals of metallic atoms and nanosheets of $\mathrm{sp}^{2}$ carbons, respectively, while the other types of carbon- dots have an undefined carbogenic structure, being in some situations a combination of crystalline structures resembling graphene layers combined with amorphous carbon. Carbon particles exhibiting a crystalline core based on a mixture of $\mathrm{sp}^{2}$ and $\mathrm{sp}^{3}$ carbons are termed carbon quantum dots (CQDs) while those composed of a mixture of primarily $\mathrm{sp}^{3}$ carbons in a disordered structure carbon nanodots (CNDs) (Cayuela et al. 2016).

The photoluminescence (PL) properties of carbon dots vary sensitively with size, and emissions are red-shifted with increasing size (Wang and $\mathrm{Hu}$ 2014b). At present, the PL 
mechanism of carbon dots (CDs) is still under debate (Zhu et al. 2015), although different researchers have attributed their emission properties to quantum confinement effect (Li et al. 2010a), surface states determined by hybridization of the carbon backbone and the connected chemical groups (Fang et al. 2011, Hu et al. 2009), fluorophores with different degrees of p-conjugation (Bourlinos et al. 2011, Mao et al. 2010b), and the recombination of electron-hole pairs localized within small $\mathrm{sp}^{2}$ carbon clusters embedded within a $\mathrm{sp}^{3}$ matrix (Srivastava and Gajbhiye 2011). Furthermore, the PL can be tuned via surface modification or via electron/energy transfer, and numerous capping strategies have been reported (Wang and $\mathrm{Hu} 2014 b)$.

CDs characteristics can be tuned by the choice of precursors during synthesis, surface passivation and functionalization. Two types of synthesis methods, top-down and bottomup, have been reported (Esteves da Silva and Gonçalves 2011). The top-down methods involve cutting carbon resources, most commonly using concentrated oxidizing acid ( $\mathrm{HNO}_{3}$ or $\mathrm{H}_{2} \mathrm{SO}_{4} / \mathrm{HNO}_{3}$ mixture) (Tao et al. 2012). The bottom-up synthesis methods consist of thermal carbonization and acid dehydration of suitable molecular precursors (Liu et al. 2012b); passivation and/or the functionalization may be required to create highly PL CDs with tunable fluorescent features (Cayuela et al. 2015). Carbon dots possess numerous surface reactive groups that offer the chance for surface modifications. Passivation can enhance the quantum yields (QYs) of the CDs, changing the PL emission and meeting the requirements of selected applications (Zhu et al. 2015). Different strategies have been used to functionalize carbon dots with different types of molecules; for instance, macromolecules, chelating ligands, specific oligonucleotides, and metal ion or metal-mediated DNA binding for heavy metal detection (Cayuela et al. 2016, 
Vázquez-González and Carrillo-Carrion 2014). Acid oxidative treatment, typically using $\mathrm{HNO}_{3}$, can introduce carbonyl functionalities at several carbon surfaces. These groups enhance the carbon dots water solubility, which is very important for biologically applications. Furthermore, the PL can be increased significantly by surface passivation agents, as for example, amino-terminated reagents (Baker and Baker 2010a).

As interest for both fundamental research and applications has surged dramatically in the last few years, it is inevitable CDs will find their way to natural water sources where, unlike the more well studied fullerenes and carbon nanotubes, their environmental impact and fate is mostly unknown. The presumed negligible environmental impact of carbon dots can only be confirmed if their behavior in natural systems is fully understood. However, the colloidal stability of CDs in environmentally relevant aqueous suspensions has not been investigated, and neither the effect of those conditions on their PL properties. Due to their small size and large surface area, adsorption processes are expected to play a key role in determining the physicochemical properties of CDs (Chowdhury et al. 2013). Adsorption may alter the surface characteristics of the nanoparticles so that they become dispersed and thus, mobile in aqueous media (Lanphere et al. 2013). Natural organic matter, e.g. humic acid (HA), and exopolymeric substances, e.g. proteins and polysaccharides, have been shown to adsorb onto carbon nanotubes and act as stabilization agents (Saleh et al. 2010); these macromolecules can potentially interact with CDs surfaces by $\pi-\pi$ bonding and hydrophobic effects, due to the presence of $\mathrm{C}=\mathrm{C}$ double bonds as well as aromatic rings (Dimiev et al. 2012).

CDs are well known for their colloidal stability in water suspensions at low ionic strength; their PL is stable under those conditions but generally expected to decline if aggregation 
occurs. Other matrix chemistry effects have been reported. For example, their florescence intensities were shown to be $\mathrm{pH}$-dependent, decreasing with increasing $\mathrm{pH}$ (Jelinek 2016). Furthermore, a study showed no significant change in fluorescence quenching of carbon dots by HA up to a concentration of 10 ppm (Cayuela et al. 2015), while another study reported that the quenched fluorescence intensity of surface functionalized QDs was linearly proportional to the concentration of $\mathrm{Hg}^{2+}$ under optimum conditions (VázquezGonzález and Carrillo-Carrion 2014).

In summary, there is extensive literature on inorganic nanoparticle stability in natural waters, as well as for some common carbon-based particles such as carbon nanotubes, fullerenes and a few studies on graphene oxides, but the aggregation and surface properties of carbon dots under environmental conditions have not been investigated. In view of the increasing amount of research devoted to carbon dots applications for sensing and imaging, an understanding of the colloidal stability is needed as a first step towards the prediction of environmental fate, transport and potential toxicity of these nanomaterials. It has been recognized that our knowledge on nanoparticle environmental behavior needs to be continuously revised and extended to account for new materials or novel commercial applications as they emerge, in order to accurately assess the associated risks (Troester et al. 2016).

The objective of this work is to investigate for the first time the colloidal stability of carbon dots, an emerging carbon nanomaterial, in natural water conditions (i.e. variable salinity, $\mathrm{pH}$, dissolved organic matter) and the effect of the transformations they undergo under such circumstances on their fluorescence properties. Two types of carbon dots were considered: as obtained from thermal treatment of organic molecules, and surface 
functionalized, to represent differences in their surface chemistry. The particles were synthesized and characterized with respect to size and surface chemistry. Aggregation was investigated under environmental relevant conditions and DLVO theory was applied to the modeling of particle stability.

\subsection{Materials and Methods}

\subsubsection{Synthesis of AC-dots}

Unfunctionalized or plain carbon dots (C-Dots) were synthesized following a modification of a previously published method (Wang et al. 2011b). A 70\% w/w glycerol solution (Sigma, $>99.5 \%$ ) was mixed with a $20 \%$ w/w solution of $\mathrm{NaH}_{2} \mathrm{PO}_{4}$ (Fisher Scientific) in a 10:1 volume ratio. The solution was heated for 20 minutes at $900 \mathrm{~W}$ power in a domestic microwave. The product was allowed to cool to room temperature and the C-dots resuspended by addition of water and continuous stirring until a homogenous colored suspension was obtained.

Amine capped CDs (a-CDs) were fabricated following a procedure described by (Zheng et al. 2014a). Briefly, $2.10 \mathrm{~g}$ of a $10 \mathrm{mmol}$ solution of citric acid (Sigma-Aldrich, ACS reagent, $>=99.5 \%$ ) were added to $10 \mathrm{~mL}$ of branched polyethylenimine ethylenediamine (BPEI) (Sigma-Aldrich) with an average molecular weight of $800 \mathrm{Da}$, as reported by the manufacturer. The reaction was heated to $170^{\circ} \mathrm{C}$ in a silicone oil bath (Fisher Scientific) for $2 \mathrm{hr}$. The yellow colored products were naturally cooled to room temperature followed by addition of deionized water.

The resulting solutions of both CDs and a-CDs were dialyzed with a Biotech CE dialysis membrane (Spectrum Labs, MWCO 1.0 kDa) against ultrapure water (specific resistance $18 \mathrm{M} \Omega * \mathrm{~cm}$ ) for 2 days for purification. Water was replaced every $6 \mathrm{hr}$. Finally, CDs and 
a-CDs were freeze-dried.

\subsubsection{Characterization}

The surface functional groups of the synthesized materials were investigated by FourierTransform Infrared Spectroscopy (FTIR) in a Nicolet 4700 FT-IR spectrophotometer (Thermoscientific) and the spectra collected over a wavenumber range from 400 to 4000 $\mathrm{cm}^{-1}$.

CDs and a-CDs were imaged by Transmission Electron Microscopy (TEM) in a Tecnai F30 Twin transmission electron microscope (FEI). TEM images were then analyzed using the software ImageJ to obtain the particle size distribution (Schneider et al. 2012a). In brief, the TEM images were selected and imported to ImageJ, then the particles on the images were segmented by adjusting the threshold value and sizes were analyzed by the software. At least 400 particles were measured for each type of nanoparticle.

Hydrodynamic diameter (Dh) and zeta potential were measured with a ZetaSizer Nano ZS (Malvern Instruments, Worcestershire, U.K.), using a monochromatic coherent He-Ne laser with a fixed wavelength of $633 \mathrm{~nm}$. Laser Doppler velocimetry was applied to characterize the electrophoretic mobility (EPM) of the nanomaterials in the various electrolyte solutions. Measured EPMs were converted to zeta-potential using the Smoluchowski equation (Elimelech et al. 2013).

$U=\frac{\varepsilon \zeta}{\mu}$

Equation 3.1

where $U$ is the electrophoretic mobility, $\varepsilon$ is the dielectric constant of the solution, $\mu$ is its viscosity, and $\zeta$ is the zeta potential. Disposable folded capillary cells were employed. 
The hydrodynamic properties of carbon dots were measured under a wide range of solution compositions, including monovalent and divalent electrolytes, represented by $\mathrm{NaCl}$ (reagent grade, Acros Organics) and $\mathrm{CaCl}_{2}$ (reagent grade, J.T.Baker) respectively. Humic acid (technical grade, Acros Organics) was introduced as a model of natural dissolved organic matter (NOM). The $\mathrm{pH}$ of the solution was adjusted by adding $\mathrm{HCl}$, (10mM, Fisher Scientific) or $\mathrm{NaOH}$ (10mM, Fisher Scientific) solutions.

UV-vis adsorption spectra for CDs and a-CDs were recorded using UV-vis spectrophotometer (Lab Tech, UV 8100B, USA) with scanning range 200-600 nm. Photoluminescence was measured on a Hitachi F-4500 Fluorescence Spectrophotometer, equipped with a $150 \mathrm{~W}$ Xenon lamp. Emission scans were performed at an excitation wavelength of $350 \mathrm{~nm}$. The slit width was $5 \mathrm{~nm}$ and scans were conducted in an emission wavelength range from $300 \mathrm{~nm}$ to $700 \mathrm{~nm}$ at a speed of $1,200 \mathrm{~nm} / \mathrm{min}$. Concentration of a-CDs were kept constant at $200 \mathrm{ppm}$ for all absorption and fluorescence experiments; CDs concentrations were $1,600 \mathrm{ppm}$ for adsorption and 52,000 ppm for fluorescence experiments.

\subsubsection{DLVO modeling}

The total interaction energy between particles as they approach each other $\left(V_{\text {TOTAL }}\right)$ was calculated to determine the aggregation process, as described by the traditional DLVO theory. According to this theory, the stability of colloidal particles in aqueous environments is determined by the net effect of Van der Waals interactions $\left(V_{V d W}\right)$ and electrical double layer interactions $\left(V_{E D L}\right)$.

$$
V_{\text {Total }}=V_{E D L}+V_{v d w}
$$

Equation 3.2 
Nanomaterials were modeled as spherical particles with diameter equal to their hydrodynamic diameter. Since dispersion forces are electromagnetic in character, they are subject to a retardation effect (Baker and Baker 2010a, Chowdhury et al. 2013). Thus, Van der Waals interactions were calculated with retardation (Gregory 1975, 1981) by equation 3:

$V_{v d w}=-\frac{A_{i w i} a}{12 h}\left[\left(1+\frac{14 h}{\lambda}\right)\right]^{-1}$

Equation 3.3

where $a$ is the particle radius $(\mathrm{nm}) ; h$ is the surface-to-surface distance between two particles $(\mathrm{nm})$; $\mathrm{A}_{\mathrm{iwi}}$ is the Hamaker constant, $6.26 \times 10^{-21} \mathrm{~J}$ was employed in this study (Feriancikova and $\mathrm{Xu} 2012$ ), $\lambda$ is the characteristic wavelength of the dielectric and usually assumed to be $100 \mathrm{~nm}$.

Electrostatic double layer interactions were calculated using the following expressions:

$V_{E D L}=2 \pi \varepsilon_{0} \varepsilon a \psi^{2} \ln [1+\exp (-k h)]$

Equation 3.4

$n_{\infty}=1000 \times N_{A} \times C_{s}$

Equation 3.5

$\gamma=\operatorname{Tanh}\left(\frac{Z e \psi}{4 k T}\right)$

Equation 3.6

where $a_{P}$ is the radius (nm); $h$ is the surface-to-surface distance between two particles $(\mathrm{nm}) ; n_{\infty}$ is bulk number density of ions which is calculated according to equation (5) where $C S$ is the electrolyte molar concentration and $N_{A}$ is Avogadro's constant; $k$ is Boltzmann constant; and $T$ is absolute temperature.

The parameter $\gamma$ is calculated according to equation (6), where $Z$ is the charge of the ions; $\psi$ is the experimental zeta potential; $e$ is the electron charge; and $K$ is the Debye length 
$\left(\mathrm{m}^{-1}\right)$ as calculated below:

$k=2.32 \times 10^{9} \sqrt{\sum C_{i} z_{i}^{2}}$

Equation 3.7

where $C_{i}$ is ion concentration $\left(\mathrm{mol} \mathrm{m}^{-3}\right) ; z_{i}$ the valence of ion $i$ including sign of charge. The application of (5) is limited to for $h<<a_{P}$ and $K a_{P}>5$.

\subsection{Results and Discussions}

The size, morphology and structure of the nanoparticles were investigated by TEM; the corresponding images of a-CDs and CDs are shown in Figure 3.1 (a) and (c). The image analysis (Figure 3.1 (b) and (d)) reveled an average particles size of $5.1 \mathrm{~nm}$ for a-CDs

and $1.8 \mathrm{~nm}$ for CDs with size ranges between $1 \mathrm{~nm}$ to $9 \mathrm{~nm}$ and $1 \mathrm{~nm}$ to $3 \mathrm{~nm}$ respectively, which is in good agreement with previous reports in the literature (Wang et al. 2011a). 

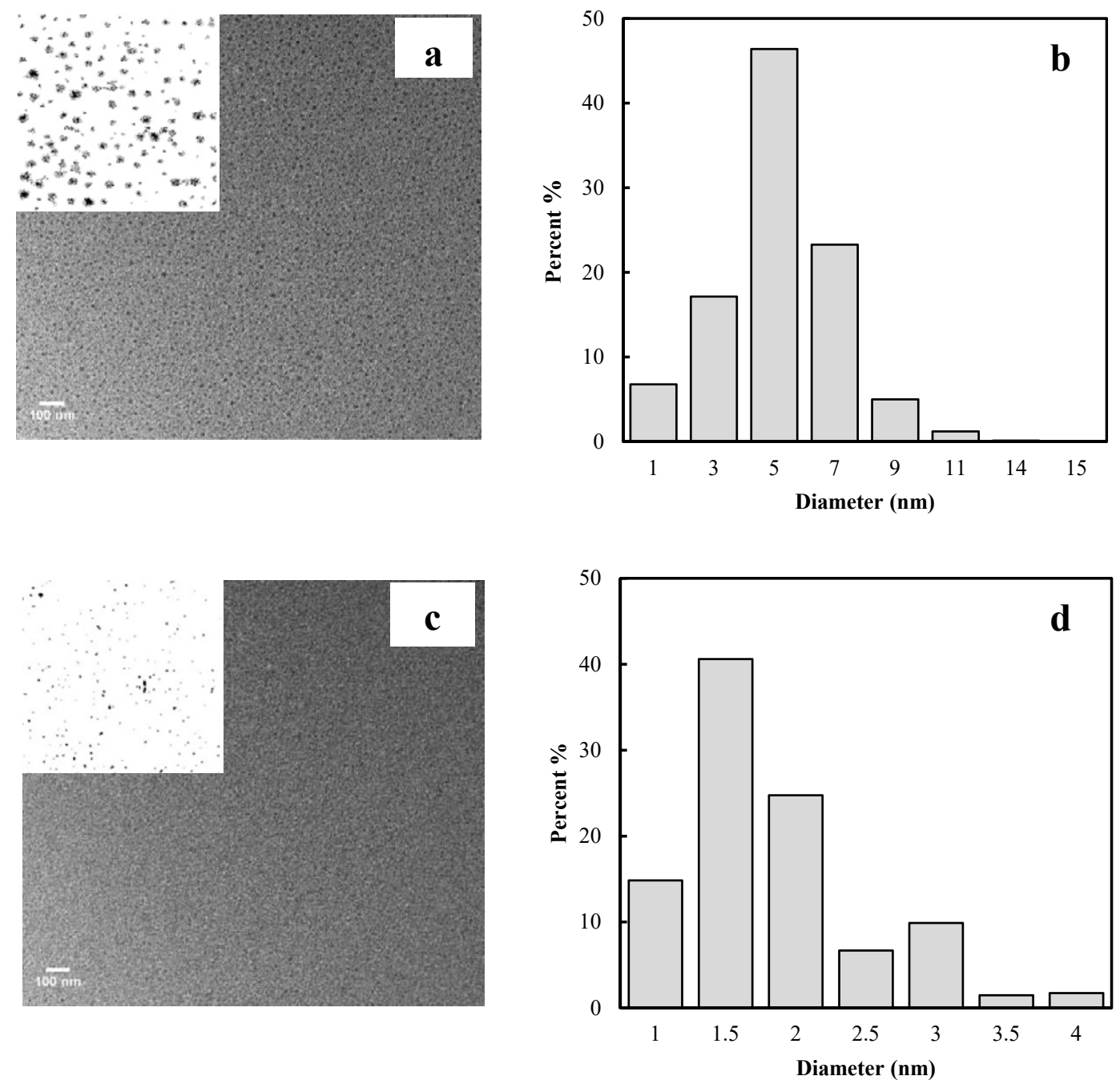

Figure 3.1 (a), (c) Representative TEM image of a-CDs and CDs: insets represent the segmented particles after subtracting the background using ImageJ software. (b), (d) Size distribution of a-CDs and CDs, as percent number of particles. 

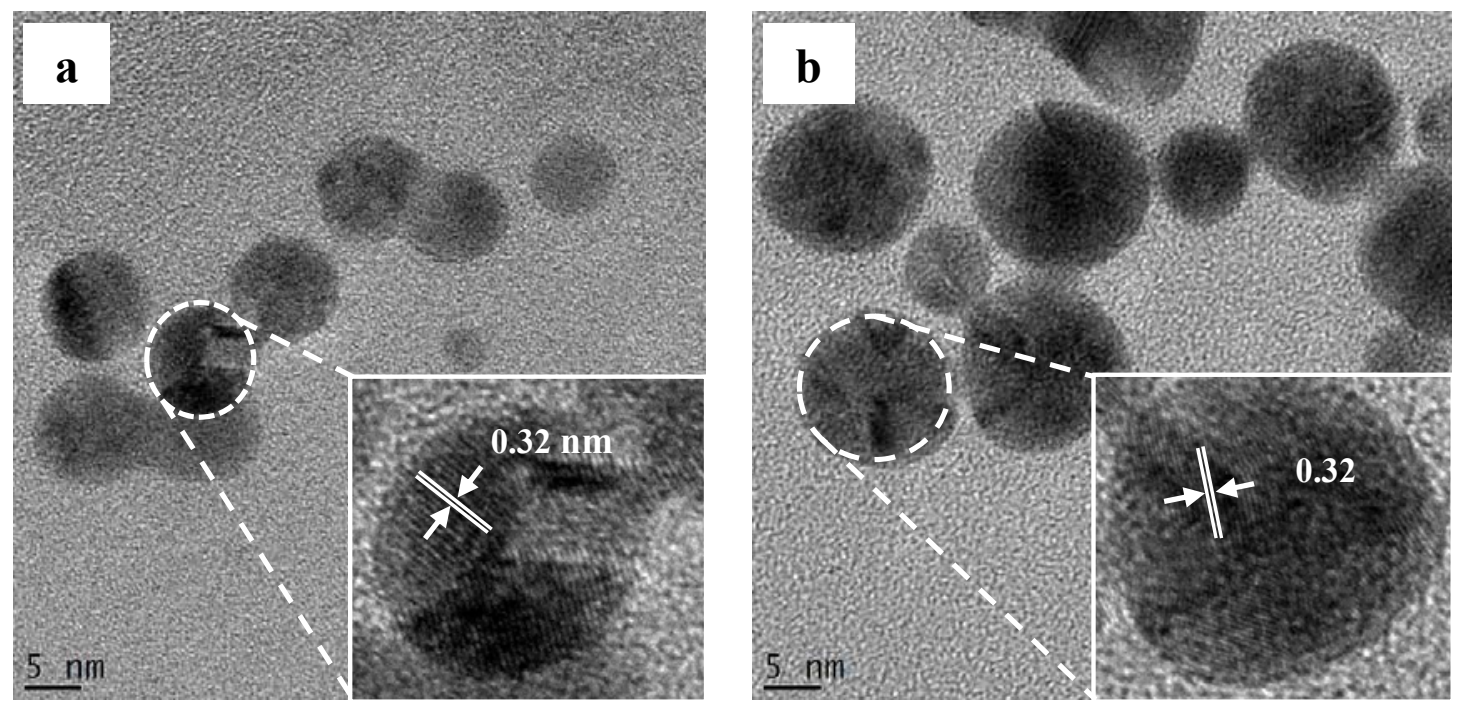

Figure 3.2 (a), (b) Representative HRTEM images of a-CDs and CDs respectively with lattice parameters of $0.32 \mathrm{~nm}$.

Figure 3.2 (a) and (b) represent HRTEM images for CDs and a-CDs respectively. The images reveal the lattice spacing of $0.32 \mathrm{~nm}$ for both type of carbon dots, which agree with (002) spacing of graphitic carbon (Tang et al. 2013).

The surface chemistry was investigated by FTIR; the spectra are presented in Figure 3.3. The FTIR spectrum of CDs (Figure 3.3b) showed the existence O-H, C=C, epoxy groups at the positions of 3223,1450 and 1058, respectively. In contrast, inspection of the a-CDs spectrum suggests abundant presence of amino groups on the surface (Figure 3.3a). The stretching vibrations of $\mathrm{N}-\mathrm{H}$ at $3286 \mathrm{~cm}^{-1}, \mathrm{C}-\mathrm{H}$ at $2958 \mathrm{~cm}^{-1}$ and $2819 \mathrm{~cm}^{-1}$ were observed in the high wavenumber region. Two strong peaks at $1658 \mathrm{~cm}^{-1}$ and $1570 \mathrm{~cm}^{-1}$ contributed to the amide linkage formed. The adsorption bands of $\mathrm{C}=\mathrm{C}, \mathrm{C}=\mathrm{N}, \mathrm{C}=\mathrm{C}-\mathrm{O}$ and epoxy groups were identified at $1466,1362,1300,1111 \mathrm{~cm}^{-1}$, respectively. 

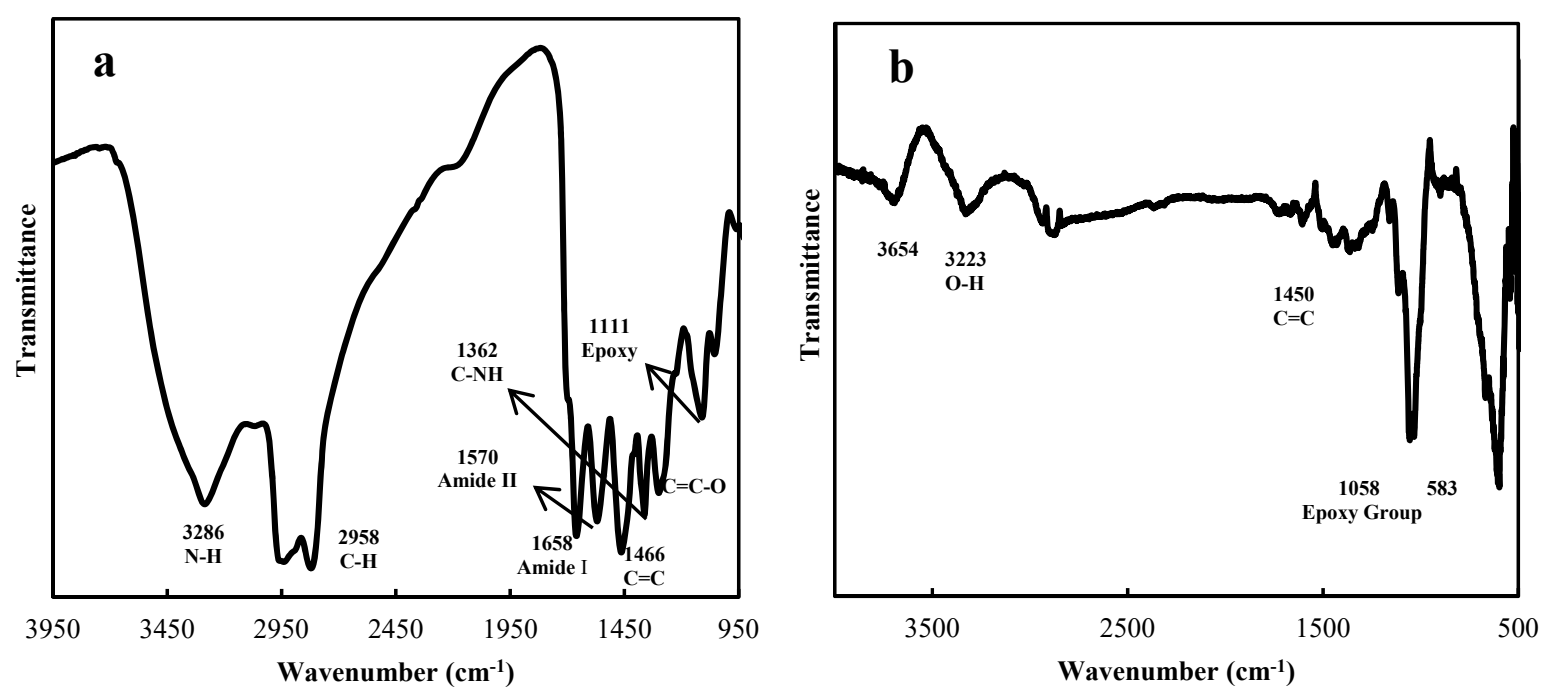

Figure 3.3 FT-IR spectra of: (a) a-CDs. (b) CDs

As prepared, both nanoparticles formed stable colored suspensions in ultrapure water due to their highly hydrophilic nature. The resulting $\mathrm{pH}$ was 5.7 and 9.8 for the CDs and aCDs suspensions, respectively. This result indicates that the basic functional groups were exposed on the surface of the nanoparticles. Under these conditions, their hydrodynamic diameter was determined to be $1.1 \pm 0.4 \mathrm{~nm}(\mathrm{CDs})$ and $1.9 \pm 0.1 \mathrm{~nm}(\mathrm{a}-\mathrm{CDs})$ and their surface charge, given by the zeta potential, was $-11.4 \pm 3.5 \mathrm{mV}(\mathrm{CDs})$ and $6.9 \pm 0.6 \mathrm{mV}$ (a-CDs). It is important to note that the mean particle size measured by dynamic light scattering (DLS) in solution was smaller than the TEM based determination. DLS size measurements are usually expected to yield larger values than those from microscopy images, given that they represent hydrodynamic diameters, which led to the conclusion that there was some degree of aggregation during the drying stage in the preparation of TEM samples, even though extreme care was exercised to avoid it with highly diluted particle suspensions and different evaporation methods. As sample drops were deposited 
on the carbon mesh, carbon dots possibly accumulated in one spot, producing an image compatible with larger particle size.

The combined effect of small particle size and surface charge was responsible for the easy dispersion and remarkable stability of carbon dots suspensions. Moreover, suspensions prepared in the laboratory remained free of sediments for as long as 2 months.

\subsection{Influence of ionic strength}

Figure 3.4 summarizes the effect of ionic strength (IS) and electrolyte type $\left(\mathrm{NaCl}, \mathrm{CaCl}_{2}\right)$ on zeta potential of $\mathrm{CDs}$ and a-CDs. $\mathrm{CDs}$ were negatively charged for all $\mathrm{NaCl}$ and $\mathrm{CaCl}_{2}$ concentrations considered; in contrast, a-CDs exhibited positively charged surfaces. Fig. 4 (a) shows that zeta potential values of CDs became less negative from -11.4 \pm 3.5 to $3.9 \pm 0.5 \mathrm{mV}$, as IS of the $\mathrm{NaCl}$ solution increased from 1 to $100 \mathrm{mM}$. This less negative surface charge can be attributed to the electrical double layer compression, as predicted by classical colloidal theory (Elimelech et al. 2013, Verwey 1947). As IS increases and the thickness of the electrical double layer diminishes, a faster decay of the potential energy of interaction with distance from the surface occurs, placing the shear plane, where zeta potential is measured, at a location of lower absolute potential value. In contrast, IS variation caused a moderate zeta potential increase for the already positively charged a-CDs, followed by a decrease at higher electrolyte concentrations. The zeta potential values of a-CDs increased from $5.4 \pm 0.6$ to $15.4 \pm 0.6 \mathrm{mV}$, as $\mathrm{IS}(\mathrm{NaCl})$ increased from 1 to $10 \mathrm{mM}$ and decreased to $8.64 \pm 0.6 \mathrm{mV}$ at $50 \mathrm{mM}$ level. The effect was more noticeable for $\mathrm{Na}^{+}$(monovalent) than $\mathrm{Ca}^{2+}$ (divalent) cations. This observation suggests the occurrence of specific interactions between the dissolved electrolytes and the 
nanoparticles, that gave rise to an apparent higher surface charge; however, this phenomenon was overcome by the double layer compression at higher ionic strength conditions, leading to a reduced magnitude of the zeta potential.

Figure 3 summarizes the effect of ionic strength (IS) and electrolyte type $\left(\mathrm{NaCl}, \mathrm{CaCl}_{2}\right)$ on zeta potential of C-dots and AC-dots. 

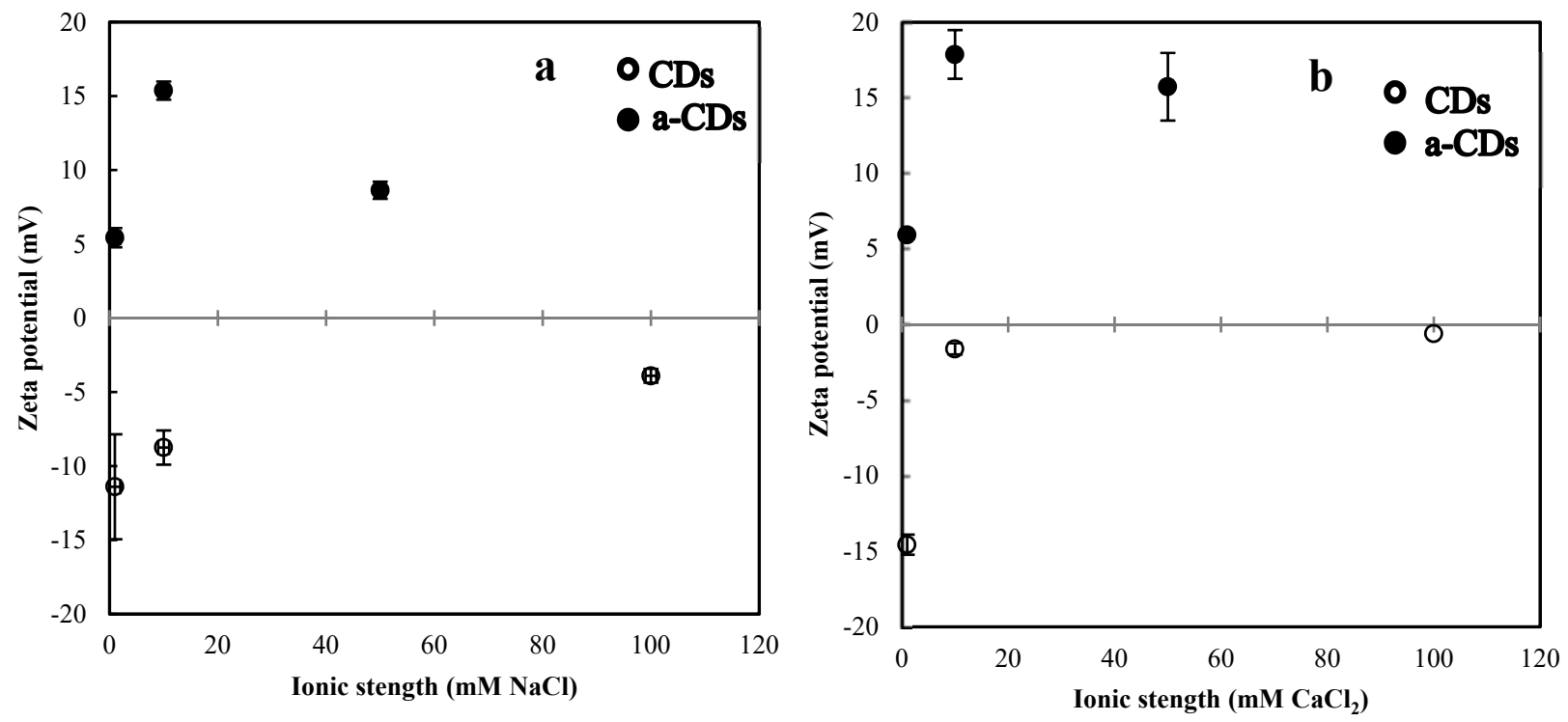

Figure 3.4 Zeta potential of $\mathrm{CDs}(\mathrm{pH}=5.7)$ and a-CDs $(\mathrm{pH}=9.7)$ as a function of ionic strength $(\mathrm{NaCl}$ and $\left.\mathrm{CaCl}_{2}\right)$.
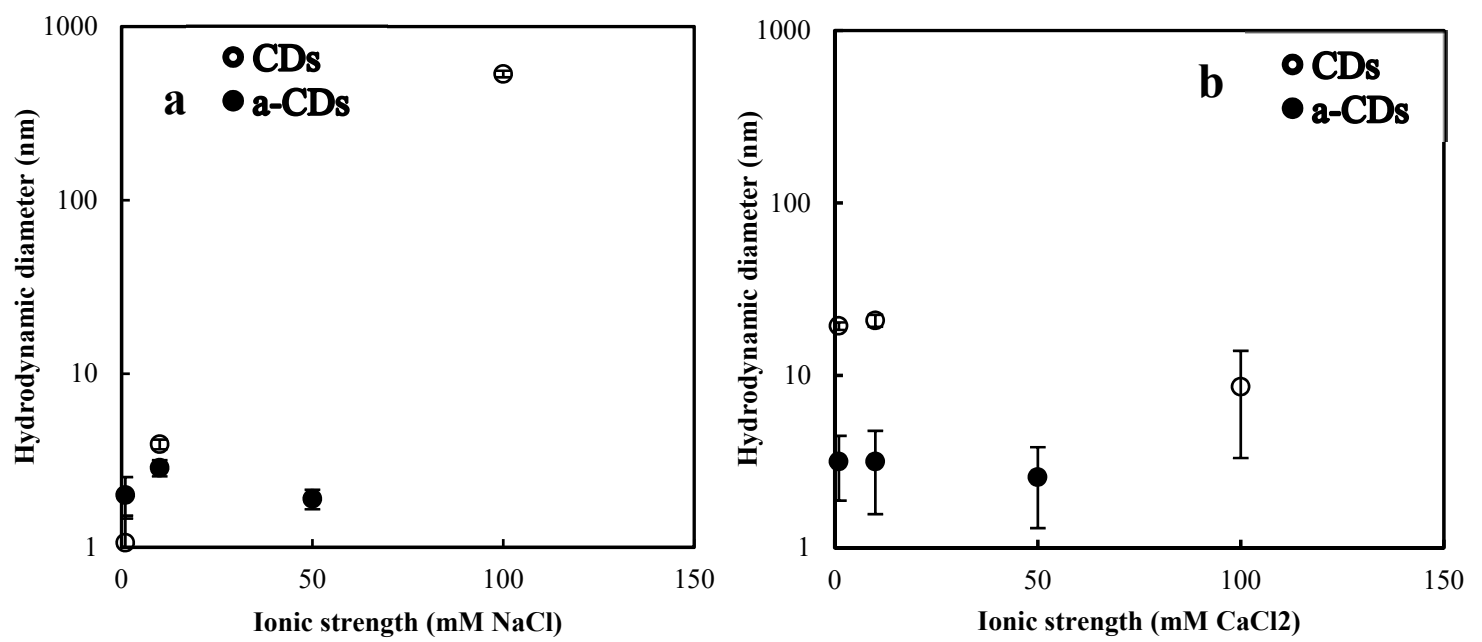

Figure 3.5 Hydrodynamic diameter of $\mathrm{CDs}(\mathrm{pH}=5.7)$, a-dots $(\mathrm{pH}=9.7)$ as a function of ionic strength $(\mathrm{NaCl}$ and $\mathrm{CaCl}_{2}$ )

Figure 3.5 (a) shows a significant increase of CDs hydrophilic diameter $\left(D_{h}\right)$ from $1.1 \pm$ 
0.5 to $533.2 \pm 23.1 \mathrm{~nm}$ suspended in $\mathrm{NaCl}$ solutions. The high $D_{h}$ at $100 \mathrm{mM}$ is in coincidence with the low absolute zeta potential value. The presence of $\mathrm{NaCl}$ did not affect the $D_{h}$ of a-CDs appreciably as shown in Fig. 5(b) and the $D_{h}$ remained approximately constant around $2 \mathrm{~nm}$.

In most natural freshwater bodies, ionic strength is below $10 \mathrm{mM}$ (Chowdhury et al. 2013), which indicates that aggregation is not expected for CDs in those aquatic environments; however, seawater and groundwater conditions may lead to substantial size increase.

The zeta potential and hydrodynamic characterization of CDs, and a-CDs in the presence of a divalent cation $\left(\mathrm{Ca}^{2+}\right)$ are shown in Figure 3.4 (b) and Figure 3.5 (b), respectively. $\mathrm{Ca}^{2+}$ influenced both zeta potential and hydrodynamic diameter more noticeable than the monovalent $\mathrm{Na}^{+}$. Figure 3.4 (b) shows that zeta potential values of CDs are significantly altered by $\mathrm{Ca}^{2+}$, as they became less negative from $-14.8 \pm 0.7$ to $-0.6 \pm 0.04 \mathrm{mV}$, as IS $\left(\mathrm{CaCl}_{2}\right)$ increased from 1 to $100 \mathrm{mM}$. The almost null zeta potential observed may be attributed to specific adsorption of $\mathrm{Ca}^{2+}$ on the negatively charged surface groups of the CDs. On the other hand, the zeta potential of a-CDs increased from $5.9 \pm 0.3$ to $17.9 \pm$ $1.6 \mathrm{mV}$, as the IS $\left(\mathrm{CaCl}_{2}\right)$ increased from 1 to $10 \mathrm{mM}$ but remained virtually constant from $10 \mathrm{mM}$ to $50 \mathrm{mM}$.

Figure $3.5 \mathrm{~b}$ shows the $D_{h}$ of CDs increased to $19.3 \pm 1.0,20.8 \pm 1.7$, and $8.6 \pm 5.3 \mathrm{~nm}$ in presence of 1,10 , and $100 \mathrm{mM}$ IS given by $\mathrm{CaCl}_{2}$ respectively. The stability behavior does not appear to be affected by the change in surface charge of the nanoparticles under those conditions. In comparison to the small diameters of the nanoparticles, hydrated $\mathrm{Ca}^{2+}$ ions have a non-negligible size of $1.2 \mathrm{~nm}$ (Dubin 2003), the specific adsorption on 
CDs may increase the size of nanoparticles and cause moderate aggregation among them at low concentrations, while tend to repulse each other at higher concentrations. Similar to $\mathrm{Na}^{+}$, no effects of $\mathrm{Ca}^{2+}$ on a-CDs were observed, and $D_{h}$ remained constant around 3 $\mathrm{nm}$. The observed behavior of the BPEI coated a-CDs were strikingly different from CDs under these conditions, probably due to radical differences in surface chemistry given by the branched amino groups on the surface. a-CDs exhibit a very good stability in the presence of dissolved salts for all the conditions investigated.

\subsection{Influence of pH}

Our measurements indicated that zeta potential values of $\mathrm{CDs}$ were $\mathrm{pH}$ sensitive and they decreased with increasing $\mathrm{pH}$. Zeta potential of $\mathrm{CDs}$ remained negatively charged over a $\mathrm{pH}$ range from 2 to 11 in the presence of $\mathrm{NaCl}$ and $\mathrm{CaCl}_{2}$, and positively charged over the $\mathrm{pH}$ range from 2 to 10 for a-CDs (Figure 3.6).

In the presence of $1 \mathrm{mM}$ IS ( $\mathrm{NaCl}), \mathrm{CDs}$ ' zeta potential decreased from $-1.4 \pm 0.7$ to $35.1 \pm 2.2 \mathrm{mV}$, as $\mathrm{pH}$ increased from 3 to 11 respectively. The zeta potential decline can be related to the deprotonation of surface functional group, such as carboxyl and hydroxyl groups. Wang showed that CDs also contain hydroxyl and carboxyl functional groups, which agrees with our FTIR results (Wang et al. 2011a). DLS results show that the $\mathrm{pH}$ did not have a noticeable effect on CDs stability from $\mathrm{pH} 4$ to 11 in presence of $\mathrm{NaCl}$. The $D_{h}$ of CDs was around $1 \mathrm{~nm}$, and then increased only slightly as $\mathrm{pH}$ decreased from 4 to 2 .

When CDs were suspended with a background ionic strength of $1 \mathrm{mM}$ given by $\mathrm{CaCl}_{2}$, the zeta potential decreased from $-3.3 \pm 0.3$ to $-20.1 \pm 4.1 \mathrm{mV}$ as a $\mathrm{pH}$ increased from 3 to 11 (Figure $3.6 \mathrm{~b}$ ). The $\mathrm{CDs}$ were mostly well dispersed at $\mathrm{pH}$ 9, as evidenced by a 
measured Dh of $1 \mathrm{~nm}$, but showed slight aggregation for other $\mathrm{pHs}$ considered, when the $D_{h}$ was higher but under $40 \mathrm{~nm}$.
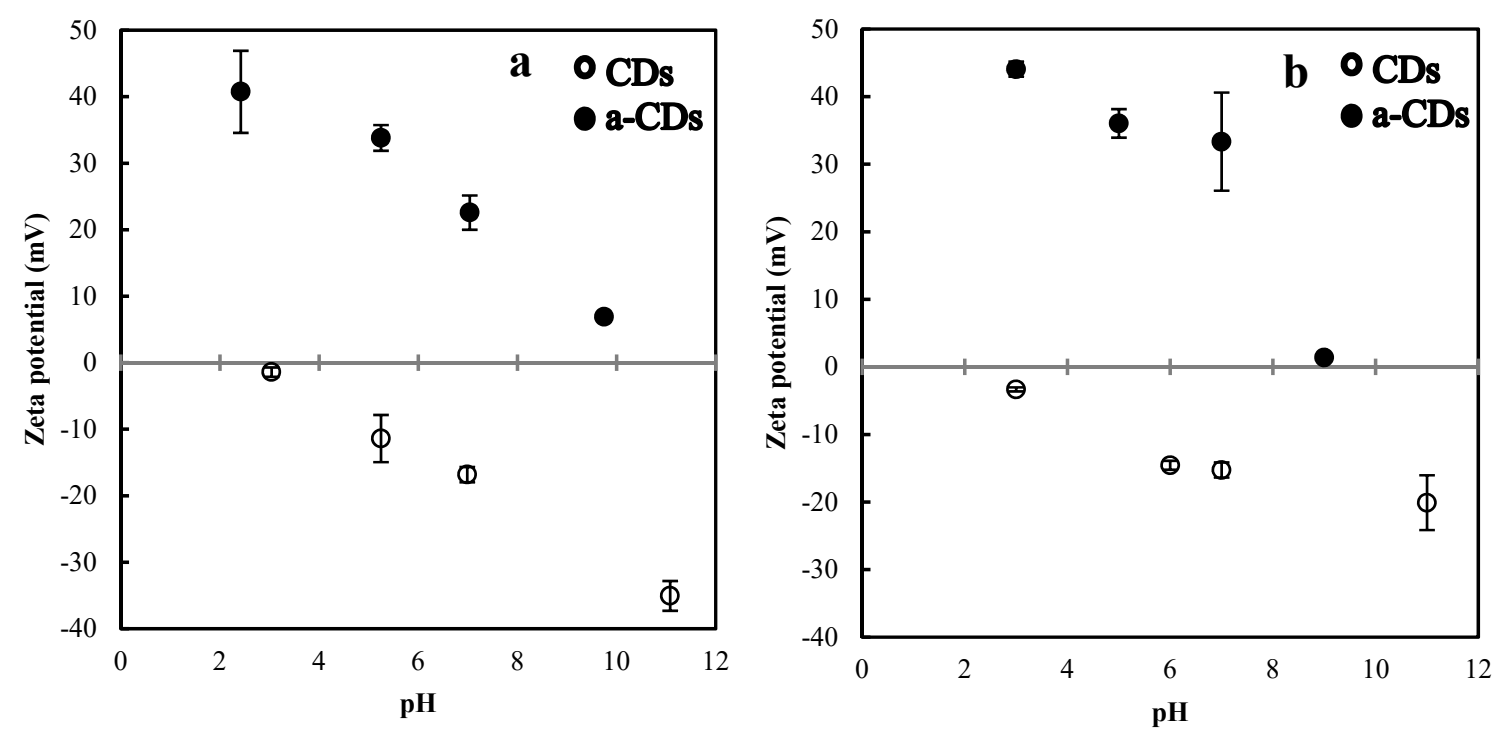

Figure 3.6 Zeta potential of CDs, a-CDs as a function of pH. (a) $1 \mathrm{mM}$ ionic strength given by $\mathrm{NaCl}$. (b) 1 $\mathrm{mM}$ ionic strength given by $\mathrm{CaCl}_{2}$.

a-CDs zeta potentials decreased from $+40.73 \pm 6.2$ to $+6.88 \pm 0.6 \mathrm{mV}$ in presence of 1 $\mathrm{mM} \mathrm{NaCl}$ as $\mathrm{pH}$ increased from 2 to 10 (Figure 3.6 a); on the other hand, the $D_{h}$ showed little to no variation (Figure 3.7 a). The a-CDs suspensions in $1 \mathrm{mM}$ ionic strength $\mathrm{CaCl}_{2}$ showed a decreasing zeta potential from $44.1 \mathrm{mV}$ to $1.4 \mathrm{mV}$ when $\mathrm{pH}$ increased from 3 to 9, and remained almost constant at higher $\mathrm{pH}$ values. The presence of amino groups indicated that $\mathrm{pH}$ significantly changed the state of charge and the properties of CDs. Amino groups have a pKa value that usually varies between 9 and 10 (Stark 1965, Wang et al. 2011a). The basic groups resulted in a higher positively charged surface due to protonation of amino groups for all $\mathrm{pH}$ range investigated. The surface charge increased with decreasing $\mathrm{pH}$, as a result of further protonation of the surface. 
The measured $D_{h}$ was small at pHs between 7 to 9 , an indication of stability, but increased at lower pHs, where nano sized aggregates were formed, mostly below $30 \mathrm{~nm}$. As discussed, divalent cations have greater influence on the aggregation of negatively charge nanomaterials compared to monovalent ions (Wagner et al. 2014). In this situation, $\mathrm{pH}$ did not appear to be a key factor affecting hydrodynamic size, but it significantly changed the surface charge due to protonation or deprotonation of surface functional groups on CDs and a-CDs (Jia et al. 2012). Moreover, size and surface charge values for both particles in ultrapure water suspensions were found to be similar to those under $1 \mathrm{mM} \mathrm{NaCl}$, within the experimental error, for the $\mathrm{pH}$ range between 2-11 (data not shown). Given the potential inaccuracy of $\mathrm{pH}$ determinations for low ionic strength solutions, i.e. ultrapure water, the $1 \mathrm{mM} \mathrm{NaCl}$ condition was used as the indifferent electrolyte condition for comparisons in this study (Davison and Woof 1985). 

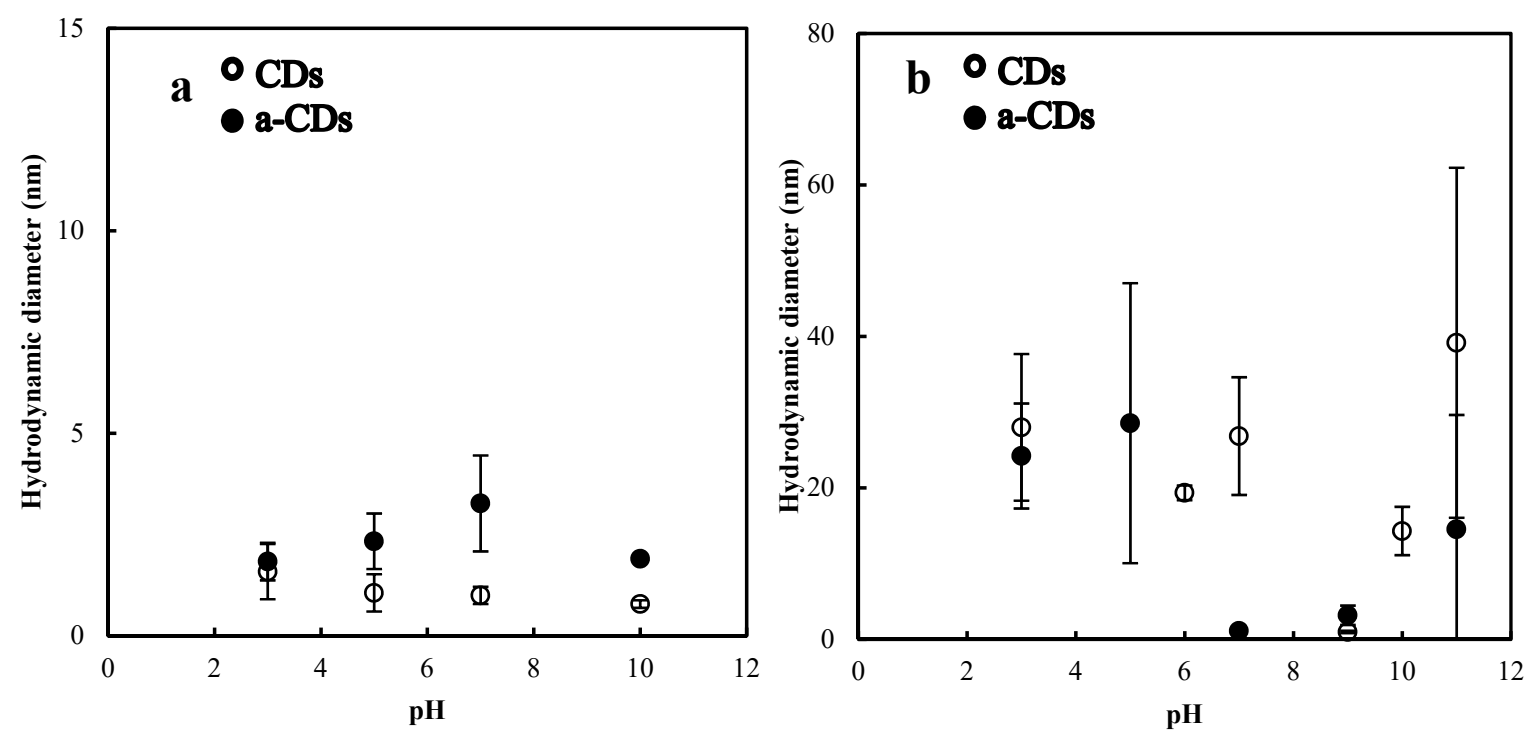

Figure 3.7 Hydrodynamic diameter of a-CDs, $\mathrm{CDs}$ as a function of $\mathrm{pH}$. (a) $1 \mathrm{mM}$ ionic strength given by NaCl. (b) $1 \mathrm{mM}$ ionic strength given by $\mathrm{CaCl}_{2}$.

\subsection{Influence of Natural Organic Matter}

The influence of dissolved natural organic matter on the stability of CDs and a-CDs was investigated in the absence of added electrolytes at circumneutral $\mathrm{pH}$. NOM has been shown to stabilize nanoparticles by inhibiting the formation of aggregates by adsorption on the surface of the nanomaterials, imparting a negative charge, and through steric effects, preventing particles to approach each other to close distances at which Van der Waals interaction become significant to overcome repulsion forces. Whether NOM results in stabilization or destabilization depends on several factors, including chemical nature of the organic molecules, concentration, and solution chemistry. Stabilization usually results from NOM forming a charged layer outside the particle, while destabilization results from particles being bridged by larger NOM molecules and/ or $\mathrm{Ca}^{2+}$ which dominate interactions between nanoparticles (Wagner et al. 2014). 
The $D_{h}$ of CDs was increased from $1.1 \mathrm{~nm}$ to $50 \sim 200 \mathrm{~nm}$ range in presence of humic acid (as shown in Figure 3.8 (a)); the $D_{h}$ of a-CDs was also increased with existing NOM, and reached $491 \mathrm{~nm}$ at the concentration of $50 \mathrm{ppm}$ HA. Surprisingly, particle sizes decreased at higher HA concentrations. This phenomenon, observed for both CDs and aCDs, can be explained by the initial bridging effect at relatively low concentration when a single HA molecule may interact with multiple nanoparticles. At increasing HA concentrations, HA molecules are present is excess and competition for surface sites resulted in HA molecules being associated with individual nanoparticle surfaces. Results are also compatible with higher adsorption affinity for a-CDs, which had a more noticeable size change. The functionalized a-CDs have abundant amine groups on the surface, which may form complexes with HA (Tang et al. 2012). Some studies have utilized this property to improve the performance of HA absorbents by functionalizing with polyethylenimine (PEI) (Deng et al. 2006, Tao et al. 2010). The absorption of HA on a-CDs may result in agglomeration and produce the size growth.

Zeta potential of CDs and a-CDs as a function of HA concentration are shown in Figure 3.8 (b). CDs zeta potential decreased from $-16.8 \pm 1.1 \mathrm{mV}$ to as low as $-58 \pm 2.7 \mathrm{mV}$, at a concentration of $50 \mathrm{ppm} \mathrm{HA}$. At neutral $\mathrm{pH}$ values, the anionic HA was adsorbed onto the surface, yielding a rather low negative zeta potential. However, a-CDs zeta potential was positive and even higher with existence of $\mathrm{HA}$, reaching values above $45 \mathrm{mV}$ for all concentrations. Zhang and Bai studied HA adsorption on chitosan-coated granules by Xray photoelectron spectroscope (XPS) and showed the formation of $-\mathrm{NH}_{3}{ }^{+} \ldots$-OOC-R or $-\mathrm{NH}_{3}{ }^{+} \ldots-\mathrm{O}-\mathrm{C}_{6} \mathrm{H}_{4}-\mathrm{R}$ surface complexes, which involved the generation of $-\mathrm{NH}_{3}{ }^{+}$as a result of interaction between the amino groups and the absorbed HA (Zhang and Bai 
2003). We postulate that this HA induced protonation of the amino groups may be the reason for the increased positive charge observed in our experiments for a-CDs in the presence of HA.
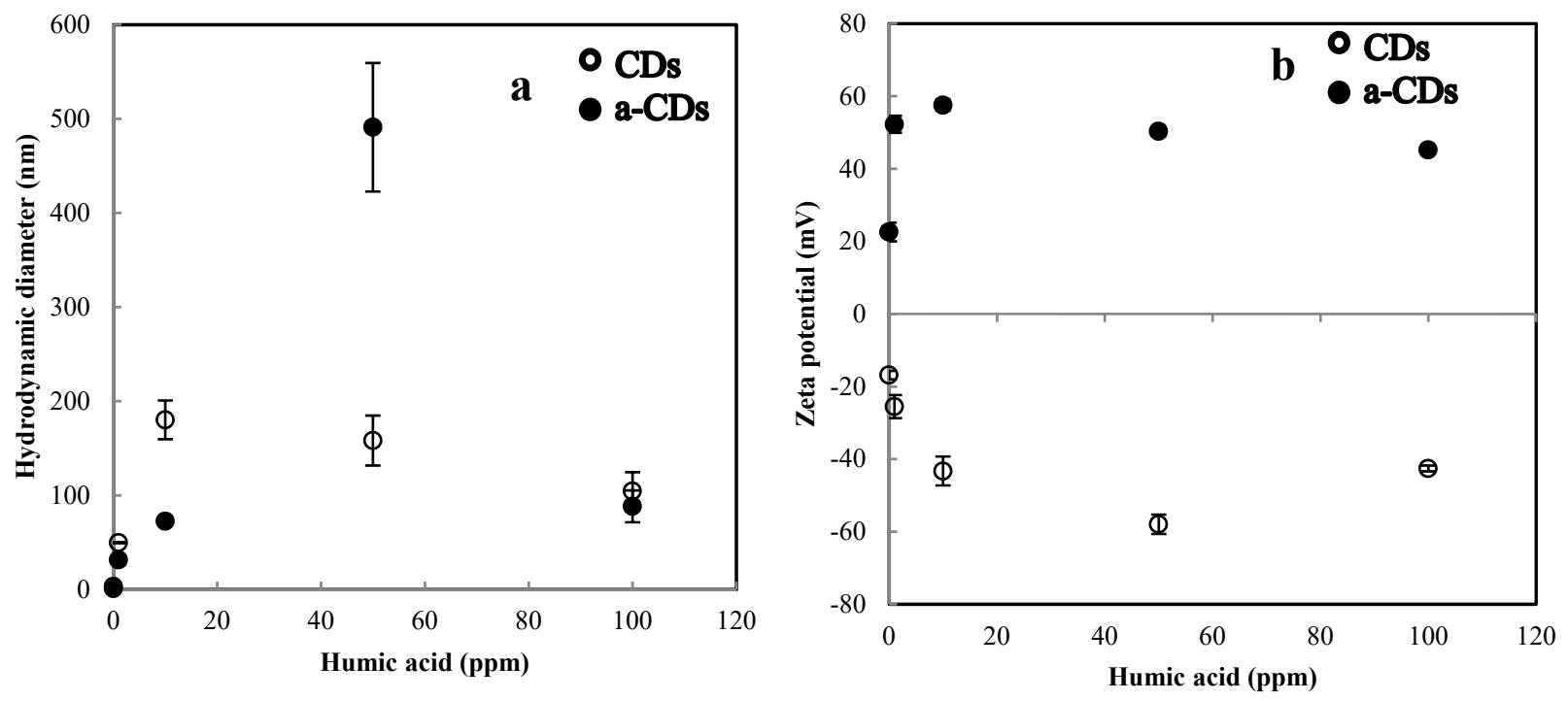

Figure 3.8 Hydrodynamic diameter (a) and zeta potential (b) of CDs, a-CDs suspended in humic acid solutions of variable concentration at neutral $\mathrm{pH}$.

\subsection{DLVO modeling}

DLVO theory was used to model CDs and a-CDs behavior. The ratio of total interaction energies to thermal energy $k T$, was calculated as a function of separation distance and plotted for $\mathrm{pH}$ ranging from 3 to 10 , and IS levels of $1 \mathrm{mM}, 10 \mathrm{mM}$ and $100 \mathrm{mM}$ for CDs (Figure 3.9) and a-CDs (Figure 3.10). Both $\mathrm{CDs}$ and a-CDs exhibited very low interaction energies for all ionic strength and $\mathrm{pH}$ conditions, with values in the same order of magnitude as the thermal energy of the system. Such a system is expected to be unstable; the extremely weak repulsion forces present will allow particles to approach to very close separation distances, where attraction predominates. Thus, particles may 
aggregate irreversibly in the deep primary energy minimum.
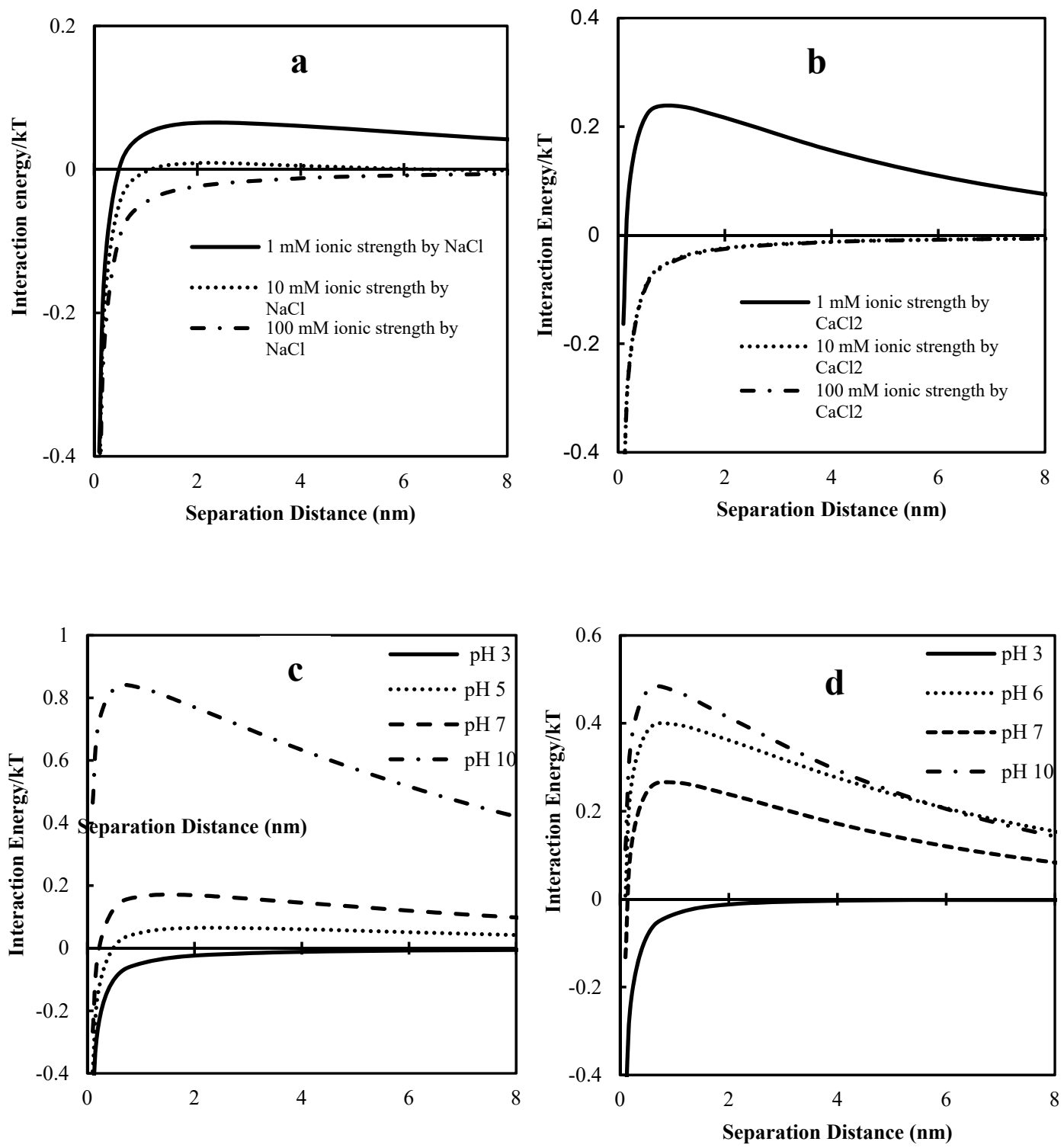

Figure 3.9 Calculated DLVO interaction energy plotted as a function of separation distance between CDs particles; effect of ionic strength and $\mathrm{pH}$ variations. (a) $\mathrm{CDs}$ with different $\mathrm{NaCl}$ concentrations. (b) $\mathrm{CDs}$ with different $\mathrm{CaCl}_{2}$ concentrations. (c) $\mathrm{CDs}$ in $1 \mathrm{mM} \mathrm{NaCl}$ with different $\mathrm{pH}$. (d) $\mathrm{CDs}$ in $1 \mathrm{mM} \mathrm{CaCl}_{2}$ with different $\mathrm{pH}$. 

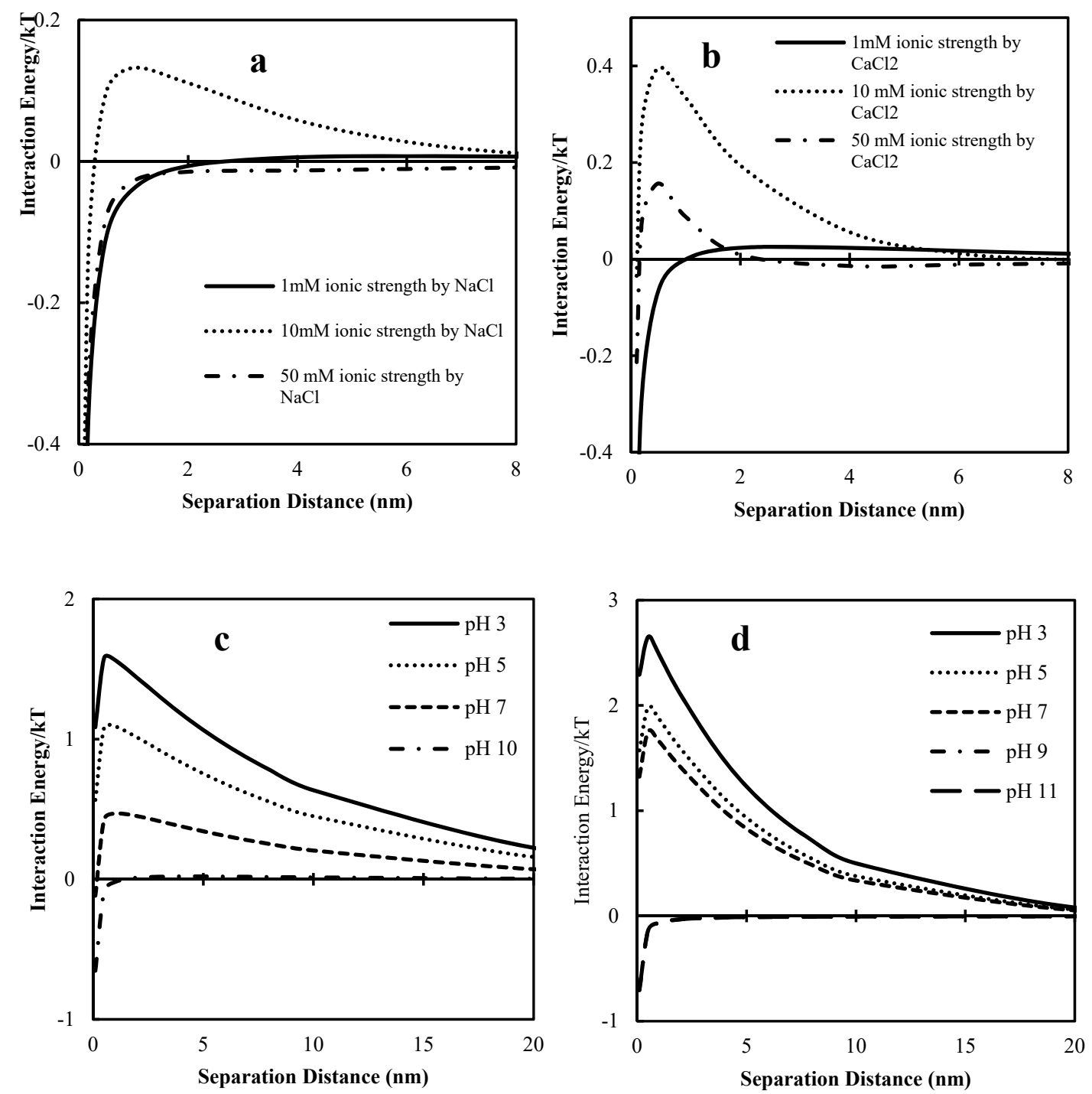

Figure 3.10 Calculated DLVO interaction energy plotted as a function of separation distance between aCDs particles; effect of ionic strength and $\mathrm{pH}$ variations. (a) a-CDs with different $\mathrm{NaCl}$ concentrations. (b) a-CDs with different $\mathrm{CaCl}_{2}$ concentrations. (c) a-CDs in $1 \mathrm{mM} \mathrm{NaCl}$ with different $\mathrm{pH}$. (d) a-CDs in $1 \mathrm{mM}$ $\mathrm{CaCl}_{2}$ with different $\mathrm{pH}$.

Pelley and Tufenkji demonstrated that particle interaction energies are very sensitive to particle size changes (Pelley and Tufenkji 2008). The height of repulsive energy barrier 
and the depth of secondary energy well increased dramatically with increasing particle diameter. The small particle size of CDs had contributed to the low calculated energies barrier, leading to instability predictions for most of the modeled conditions. However, experiments revealed the remarkable stability of carbon dots, except for the highest ionic strength level conditions. The modeling equations contemplate two effects of the addition of salts on repulsion: a decrease in zeta potential and an increase in Debye length (K); both will decrease the repulsion at a given separation distance, and hence promote aggregation.

Even if relatively accurate predictions were obtained for some conditions (i.e. CDs suspended in $\mathrm{NaCl}$, highest ionic strength conditions), the DLVO modeling was largely in disagreement with experimental results, especially for a-CDs. Classic DLVO relies exclusively in the interplay of electrostatic and Van der Waals interactions to describe colloidal stability. However, our experimental work evidenced a lack of correspondence between surface charge and size variations, which suggests a negligible influence of electrostatic repulsion in the colloidal stability of the system. In addition, the very small particle size, comparable to the Debye length and the hydrated cations diameters, further contests the applicability of the theory.

\subsection{Fluorescence}

We investigated the fluorescence emission intensity of both nanomaterials at three levels of ionic strength, given by two electrolytes: $\mathrm{NaCl}$ and $\mathrm{CaCl}_{2}$. The experiments were conducted at a constant particle concentration for each type of CD: 200 ppm for a-CDs and 52,000 ppm for CDs. Those concentrations were chosen in order to achieve high initial fluorescence emission intensities. Analysis of emission intensity changes with 
particle concentration showed that the height of the emission peak increased linearly with concentration up to the chosen particle concentrations, ruling out the potential interference of interparticle interactions, such as aggregation, in the fluorescence determinations (Figure 3.11).
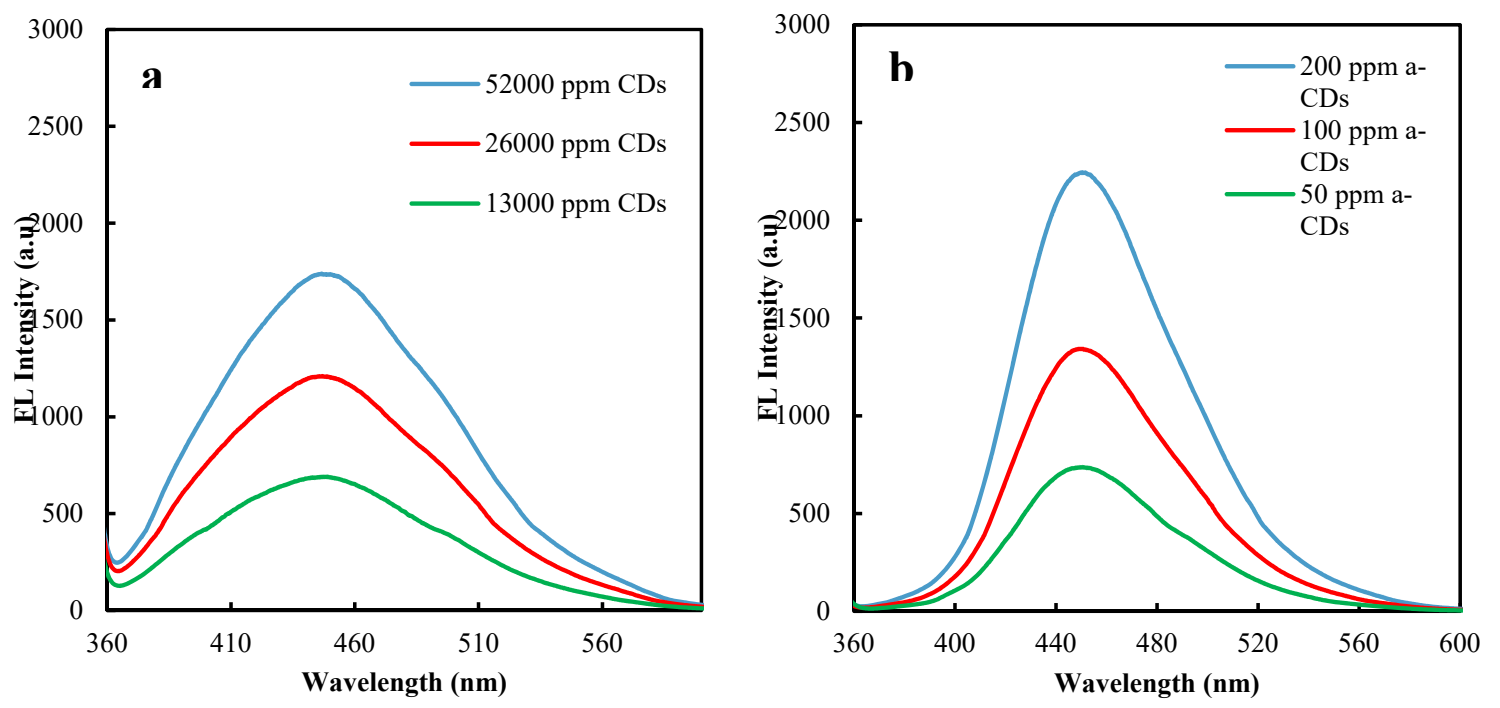

Figure 3.11 Fluorescence spectra for (a) CDs, and (b) a-CDs, at different concentrations

The ionic strength results are presented in Figure 3.12. Since aggregations negatively affects the emission, we focused our work on those ionic strength conditions in which we did not observed significant particle size growth in our DLS determinations. 

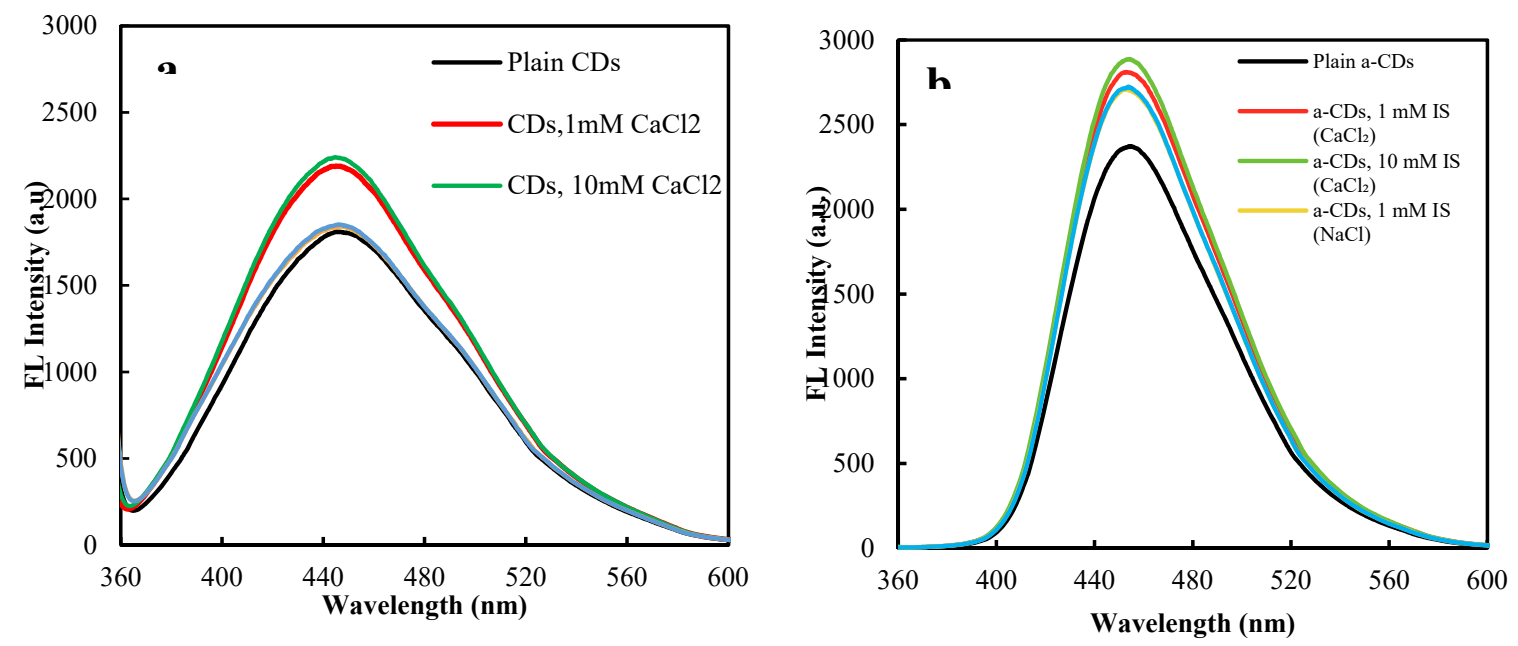

Figure 3.12 Fluorescence spectra for (a) CDs, and (b) a-CDs, at various levels of ionic strength and electrolytes.

Fluorescence emission intensity increased with ionic strength for both CDs and a-CDs in the presence of an indifferent electrolyte. The ionic strength PL behavior implies that the surface defects may serve as capturing centers for carriers, thereby resulting in correlated luminescence (Jia et al. 2012). However, different effects were observed for the suspensions prepared in $\mathrm{CaCl}_{2}$. Lower emission intensities were observed for $\mathrm{CDs}$ with increasing concentration of $\mathrm{Ca}^{2+}$ in comparison with the same ionic strength level given by the monovalent ion. However, the presence of calcium resulted in a negligible effect on the a-CDs. The specific adsorption of $\mathrm{Ca}^{2+}$ was much more noticeable on the negatively charged CDs than on the a-CDs; there was, nevertheless some adsorption as indicated by the differences in zeta potential detected for the latter, but it was not strong enough to have an influence on the fluorescence properties of the carbon dots.

We studied the influence of natural organic matter on fluorescence emission intensity of 
CDs and a-CDs, using HA as a model compound in concentrations from $1 \mathrm{ppm}$ to 100 ppm. The PL intensity of pure HA at the concentrations used in the experiment, shown in Figure 3.13, was substantially lower than that of the carbon dots. In the presence of HA, the emission intensity of CDs and a-CDs decreased with increasing concentration of the model compound. Compared to CDs, a-CDs were affected more noticeably. As discussed, HA can be adsorbed on both nanomaterials and the degree of adsorption is dependent on their surface characteristics. Therefore, functionalized a-CDs had higher affinity for adsorption of HA because of the positively charged amino groups on the surface. The better adsorption due to the formation of surface complexes between protonated amino groups and HA resulted in a larger change in emission intensity (Wagner et al. 2014). Our results suggested that PL from CDs is related to the surface states of the nanoparticles, since adsorption played a major role in controlling emission intensity. However, HA adsorption at the wavelength of emission peak for both carbon dots became significant at high HA concentration (50 ppm and $100 \mathrm{ppm}$ ), which would further contribute to the observed quenching. The negative impact by dissolved NOM on CDs and a-CDs has implications for sensing, potentially limiting the applications of CDs in high BOD streams or wastewaters (Part et al. 2016). 

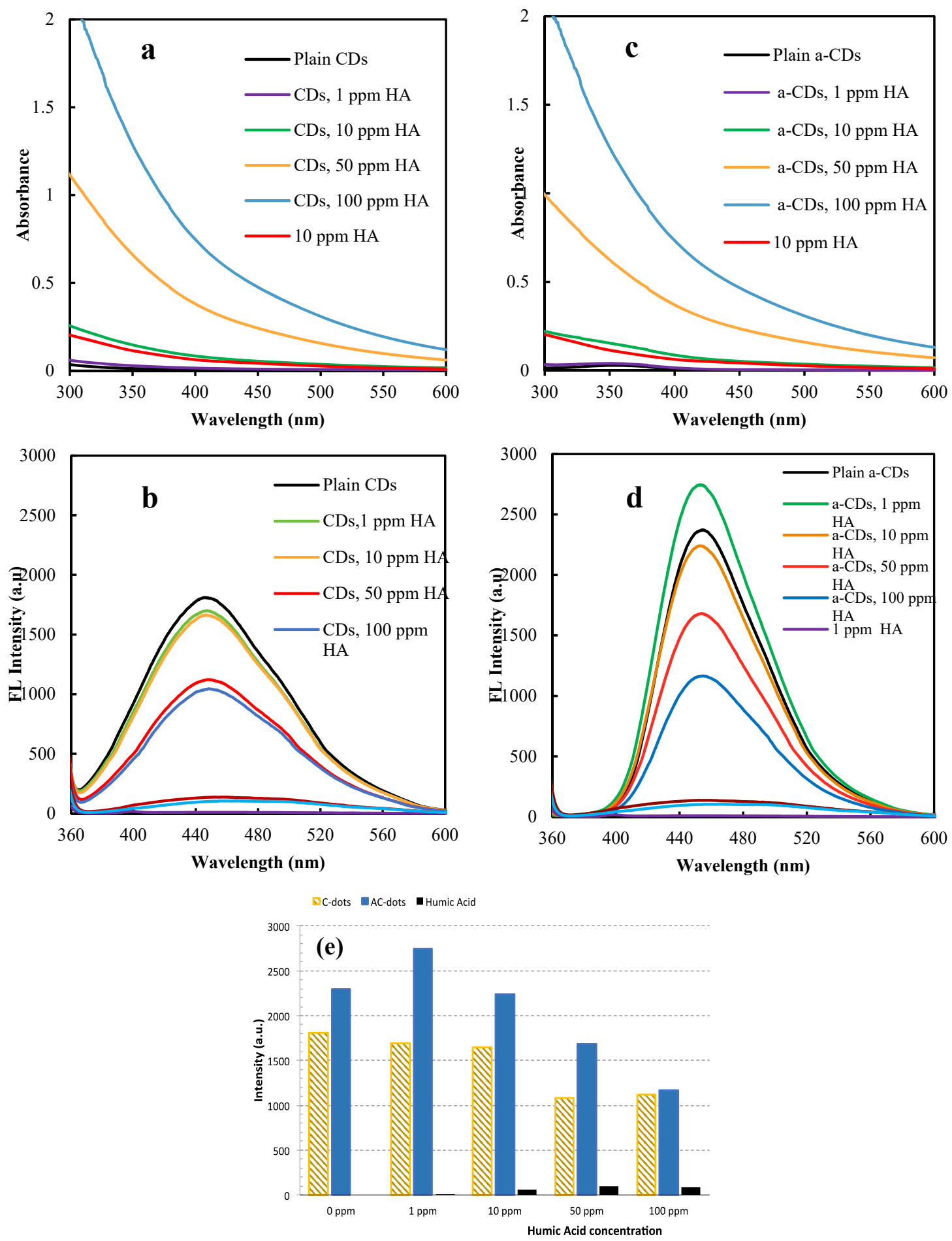

Figure 3.13 Absorption and fluorescence spectra: CDs (a) and (b); a-CDs(c) and (d), respectively, in the presence of HA; comparison of fluorescence intensity peak for CDs, a-CDs suspended in HA solutions (1ppm-100ppm) and pure HA (e). 


\subsection{Conclusions}

As prepared, CDs and a-CDs formed highly stable water suspensions and yielded a circumneutral $\mathrm{pH}$ for $\mathrm{CDs}$ and basic conditions for a-CDs. Negatively charged CDs suspended in $\mathrm{NaCl}$ solutions exhibited behavior compatible with classical colloidal stability principles, but aggregation was inhibited in the presence of $\mathrm{CaCl}_{2}$, even for very low surface charge conditions. a-CDs remained positively charged for all conditions investigated and showed higher zeta potentials at intermediate ionic strength levels followed by a moderate charge decrease, but remained stable in suspension. This is in contrast to the general behavior observed for nanoparticles (lower surface charge and aggregation with increasing ionic strength), and hints to the importance of specific adsorption of divalent cations on carbon dots and even monovalent cations for the aminocoated particles. The surface charge of both carbon dots varies with $\mathrm{pH}$ as expected from the acidity constants of their surface functional groups. However, particle size remains fairly constant, always below $50 \mathrm{~nm}$. Although nanoparticles tend to aggregate because of their high surface energies, more strongly so for smaller particles as in the case of carbon dots, coatings (either engineered or adsorbed from the suspension media, e.g. NOM) may hinder aggregation. Aggregation is expected as a result of most typical concentrations of $\mathrm{Ca}^{2+}$ in surface waters regardless of the presence of NOM and under groundwater conditions, $\mathrm{Ca}^{2+}$ and NOM being key factors in nanoparticle stability, in contrast with our findings. If nanomaterials aggregate fast under natural water conditions and settle, they will impact mostly sediments and benthic organisms. We have shown in this work that carbon dots exhibit an opposite behavior, as they will most likely remain suspended in 
natural waters and will impact animals that inhabit the water column such as planktonic species, fish, and marine mammals. Moreover, high stability translates into excellent transport properties, which may lead to impacted ecosystems far from source of contamination.

Presence of HA also led to a unique response, inducing aggregation due to bridging for low to moderate concentrations and followed by stabilization at higher levels, independently of surface charge modifications. Interestingly, both positively and negatively charged particles presented an increase in the magnitude of zeta potentials with HA concentration.

Fluorescence emission intensity declines were observed for water chemistry conditions at which specific adsorption of ions or HA was suspected, suggesting surface states as the origin of the PL. However, the adsorption of HA at the peak emission wavelength for the carbon dots may have also contributed to this observation. This phenomenon may limit the application of carbon dots in sensing of natural waters. 


\section{Chapter 4. Molecularly imprinted polymers labeled with amino- functionalized carbon dots for fluorescent determination of 2,4- dinitrotoluene}

The widespread application of DNT in the manufacturing of a diverse set of chemicals has resulted in substantial contamination of soil and water (Xu and Jing 2012). DNT is a precursor and an inevitable byproduct of the manufacturing process of 2,4,6trinitrotoluene (TNT), an explosive used worldwide. DNT is also involved in the production of polyurethane foam products, dyes, plastics, plasticizers, deterrent coatings, and burn rate modifiers for propellants (Lent et al. 2012). DNT is commonly found in surface water, groundwater and soils near former ammunition factories and facilities that manufacture or process DNT (Reddy et al. 2014). The moderately soluble DNT has low volatility and demonstrated toxicity to the hematopoietic, cardiovascular, nervous, and reproductive systems of humans (Lent et al. 2012, Tchounwou et al. 2003). Therefore, the detection of DNT is of utmost importance, and a significant amount of effort has been devoted to the development of suitable sensors, both in gas and liquid phase (Bosco et al. 2012, Wang et al. 2016). Optical detection techniques, colorimetric or fluorimetric based, have the advantage that they can be portable and used in-situ. Fluorescence sensors are especially suited for detection of nitroaromatic compounds such as DNT, due to their ability to quench emission of excited species (Goodpaster and McGuffin 2001b). Fluorescence based detectors for DNT have been reported using fluorescent conjugated polymers, hybrids composed of inorganic materials functionalized with an organic fluorophore, and quantum dots (Q-dots) (Peveler et al. 2016). The latter has shown advantages over the previous approaches, as these nanoparticles do not suffer from 
instability with respect to temperature, $\mathrm{pH}$, and photobleaching that characterizes the organic-based detection methods (Stringer et al. 2010). Although the exact chemical composition of Q-dots varies, they are typically made of cadmium selenide or sulfide (Zhang et al. 2011b), which have raised questions regarding their toxicity.

Even though fluorimetric based sensing techniques are very sensitive, they lack selectivity. One strategy to increase the selectivity of the sensing devices is the capture of the target molecules by Molecularly Imprinted Polymers (MIPs) (McCluskey et al. 2007b). MIPs are specialty polymers generated via the interaction of functional monomers, a target molecule and a cross linking agent. The template participates in the polymerization process and then is removed from the polymer leaving behind a cavity, complementary in size, shape and electronic properties to the target molecule, which becomes a highly specific capture site. MIPs are robust and can be designed to be insoluble in the media of interest (Wulff 2002). Fluorescent sensors based on Q-dots labeled MIPs have been developed for nitroaromatic explosives like DNT and TNT in aqueous phase (Xu et al. 2013), but the loss of nanoparticles or leaching of toxic heavy metals may result in serious environmental pollution so that the use of CdTe Q-dots is not suitable for an aquatic environment.

Carbon dots (C-dots) are a relatively new class of carbon nanomaterials, with sizes below $10 \mathrm{~nm}$ (Baker and Baker 2010a). Compared to traditional semiconductor Q-dots and organic dyes, photo luminescent C-dots are superior in terms of high aqueous stability, robust chemical inertness, easy functionalization, high resistance to photobleaching, low toxicity and good biocompatibility (Baker and Baker 2010a). As a result, much attention has also been paid to their potential application in biological labeling, bioimaging, and 
drug delivery (Wang et al. 2011d). During the past few years, much progress has been achieved in the synthesis, properties and application of C-dots (Shen et al. 2012b, Wang and $\mathrm{Hu} 2014 \mathrm{~b}$ ). Several low cost and environmentally benign chemical approaches have been developed for the preparation of C-dots (Wang et al. 2011b). Given their strong and tunable photoluminescence, C-dots have been successfully applied in sensing, energy conversion and catalysis (Li et al. 2015). Among them, functionalized C-dots have been synthesized through one step hydrothermal carbonization of chitosan or citric acid at mild temperature (Dong et al. 2012b, Yang et al. 2012b); besides, surface functionalization of C-dots provides an effective bridge to connect them to other systems (Ding et al. 2014). MIPs as sensors combining functionalized C-dots have been reported for recognition of Bisphenol A, 4-nitrophenol, glucose and nicotinic acid (Hao et al. 2016, Liu et al. 2016, Wang et al. 2015b, Zuo et al. 2015). However, all these reported sensors were in the form of nanoparticles or microgels, and may not be easily recovered for recycling and reuse, or suitable for in-situ testing.

In this work, we fabricated highly porous molecularly imprinted poly(methyl acrylate-coacrylic acid) (PMAAA) films to solve the aforementioned disadvantages of previously reported sensors. The porosity and surface area of the films were provided by the bulk polymerization of the monomer solution and the template molecule in the pore space of a silica colloidal crystal. AC-dots were covalently bonded to the polymer by linking amino groups and carboxyl groups with N-hydroxysuccinimide (NHS) and $\mathrm{N}-(3-$ Dimethylaminopropyl)-N'-ethylcarbodiimide hydrochloride (EDC) as coupling reagents. Compared with physical attachment or simple coating approaches to C-dots labeled sensors, the strong covalent bonding of the nanoparticles was expected to provide 
superior durability, avoiding the loss of nanoparticles and ensuring fluorescence stability. The easy detection was based on the amount of captured DNT molecules through fluorescence quenching.

\subsection{Materials and Method}

\subsubsection{Materials}

Chemicals were purchased from Sigma-Aldrich (https://www.sigmaaldrich.com/unitedstates.html) and used without further purification; tetraethoxysilane (TEOS) (98\%), ammonia solution ( $25 \%$ in water), ethanol (200 proof, ACS reagent, 99.5\%), acrylic acid (AA) (99\%), methyl acrylate (MA) (99\%), ethylene glycol dimethacrylate (EGDMA) (98\%), 2,2'-azobisisobutyronitrile (AIBN) (98\%), 2,4-dinitrotoluene (DNT) (97\%), phenol, 4-nitrophenol (4-NP) (99\%), and dinitrophenol (DNP) (98\%), hydrofluoric acid (HF) (48\%), citric acid (CA) (99.5\%), branched polyethylenimine (BPEI), Nhydroxysuccinimide (NHS), and N-(3-Dimethylaminopropyl)-N'-ethylcarbodiimide hydrochloride (EDC).

\subsubsection{Synthesis of the colloidal crystals and MIPs}

The monodisperse silica particles were synthesized following the Stöber method (Stöber

et al. 1968). TEOS $(8.3 \mathrm{~mL})$ and ethanol $(200 \mathrm{~mL})$ were mixed in a round flask and stirred at $300 \mathrm{rpm}$. Then, ammonia $(18.3 \mathrm{~mL})$ was introduced and allowed to react for 12 h. Silica particles obtained were centrifuged and redispersed twice in DI water and once in ethanol for purification. Colloidal crystals were fabricated by vertical self-assembly on a glass substrate as described elsewhere (Jiang et al. 1999). In this procedure, a cleaned glass slide was vertically placed into a flask containing a $0.5 \%$ volume fraction suspension of silica particles in ethanol. After the volatilization of ethanol, the colloidal 
crystals were formed on the slide. Another slide was placed upon the colloidal crystal film and the two slides were held together. Polymerization solutions were prepared from $0.4 \mathrm{~mL}$ (4.4 mmol) AA and $0.4 \mathrm{~mL}(5.8 \mathrm{mmol}) \mathrm{MA}$ as the functional monomer (with the molar ratio of 3:4), $0.55 \mathrm{~mL}(2.9 \mathrm{mmol})$ EGDMA as the crosslinking agent, $6 \mathrm{mg}(0.04$ mmol $)$ AIBN as the initiator, $4 \mathrm{mg}(0.02 \mathrm{mmol}) \mathrm{DNT}$ as the target molecule and $0.6 \mathrm{~mL}$ ethanol as the solvent, to yield MIP-PMAAA. One end of glass assembly was put in contact with the polymerization mixture that rises by capillary forces filling the void spaces within the colloidal crystal. Polymerization was performed under UV light at wavelength of $365 \mathrm{~nm}$ for $5 \mathrm{~h}$ at room temperature. Silica particles were removed by submerging in 5\% hydrofluoric acid bath. Then, the films were soaked in ethanol in order to elute DNT, changing the solvent every half an hour for 3 hours. The fabrication of non-imprinted copolymer (NIP-PMAAA) followed a similar procedure but without the addition of DNT to the polymerization solution. Also, nonimprinted films (NIP-PAA) were prepared entirely from AA as the functional monomer using $0.8 \mathrm{~mL} \mathrm{AA}$ instead of $0.4 \mathrm{~mL}$ AA and $0.4 \mathrm{~mL} \mathrm{MA}$, for comparison between the materials.

\subsubsection{Fluorescent Labeling}

Amino functionalized carbon dots (AC-dots) were synthesized following a published method (Zheng et al. 2014b). Briefly, $2.10 \mathrm{~g}$ (10 mmol) of CA was added to $10 \mathrm{~mL}$ of BPEI. The reaction was heated in an oil bath and held at $170{ }^{\circ} \mathrm{C}$ for 2 hours. Then, the reaction flask was naturally cooled to room temperature. After water addition, the product was dialyzed by means of a cellulose ester (CE) membrane (Spectrum Laboratories, Inc., MWCO $1.0 \mathrm{kDa}$ ) against water for 2 days. The water was replaced every $6 \mathrm{~h}$, and finally, AC-dots were freeze-dried to yield a yellow solid. The porous MIP films were placed 
into a $0.033 \mathrm{mM} \mathrm{EDC} / 0.033 \mathrm{mM}$ NHS aqueous solution. After stirring for $1 \mathrm{~h}$ at room temperature, $2 \mathrm{~mL}$ solution of AC-dots with a concentration of $5 \mathrm{~g} \cdot \mathrm{L}^{-1}$ was added dropwise. The mixture was stirred for $36 \mathrm{~h}$ at room temperature in the dark to form the covalent bonding between the AC-dots and the films.

\subsubsection{Characterization of NIP-PAA and NIP-PMAAA}

The particle size distribution (PSD) of AC-dots and silica particles was analyzed using dynamic light scattering (DLS) on a Zetasizer Nano ZS instrument (Malvern Instruments). Silica particles and porous films were imaged by a FEI Quanta 600 FEG Environmental Scanning Electron Microscopy (ESEM) to investigate the pore size and morphology. Samples were attached to a metal mount using carbon tape and were coated with a thin layer of platinum to provide a conductive surface using a sputter coater (Emitech K575x). AC-dots were imaged by Transmission Electron Microscopy (TEM) in a Tecnai F30 Twin transmission electron microscope (FEI). Quantitative analysis of the images was done using ImageJ software (National Institutes of Health, NIH).

Fourier Transformed Infrared Spectroscopy (FTIR) spectrum of AC-dots was collected with a Nicolet 4700 FT-IR in order to identify the functional groups present on the surface of AC-dots. PAA and PMAAA films were characterized with FTIR and Nuclear Magnetic Resonance Spectrometry (NMR) to compare their chemical structures. The

infrared spectra of dried NIPs were obtained by preparing $\mathrm{KBr}$ pellets in Thermo Nicolet Nexus 670 FTIR. NMR sample was prepared by dissolving a few milligrams of PAA or PMAAA in $0.6 \mathrm{ml} \mathrm{CDCl} 3$; and the solution was transferred to a 5-mm diameter NMR tube. 1H NMR was obtained at $25{ }^{\circ} \mathrm{C}$ on a Bruker AV III HD $600 \mathrm{MHz}$ NMR spectrometer equipped with a $5 \mathrm{~mm}$ HCN cryo-probe and $\mathrm{Z}$ gradient coil. A total of 168 
(PAA) and 328 (PMAA) scans were collected using a 30 degree excitation pulse and a $3.7 \mathrm{~s}$ repetition delay. A line-broadening of $0.3 \mathrm{~Hz}$ was applied to the FID before Fourier transformation. The residual chloroform solvent proton at 7.25 PPM was used for chemical shift referencing.

Thermal properties of the NIP-PAA and NIP-PMAAA films were determined by the differential scanning calorimetry (DSC) technique. The analysis was carried out on a TA Instruments Q100 Differential Scanning Calorimeter. The sample ( 2 mg of a lyophilized hydrogel) was sealed into a DSC aluminum pan. An empty aluminum pan was used as reference. The sample was studied at heating rate of $5^{\circ} \mathrm{C} / \mathrm{min}$ between $40{ }^{\circ} \mathrm{C}$ to $120^{\circ} \mathrm{C}$. Swelling experiments were performed on NIP-PAA and NIP-PMAAA in phosphate buffer at three different $\mathrm{pH}$ values $(4,7$ and 10$)$ at room temperature. The polymers were swollen in solutions for $48 \mathrm{~h}$ at ambient temperature and the degree of swelling was determined gravimetrically. The percentage swelling ratio (SR) was calculated from the expression (1)

$\% S R=\left(m_{s}-m_{d}\right) / m_{d}$

Equation 4.1

where $m_{s}$ is the mass of the swollen film at equilibrium and $m_{d}$ is the mass of the freeze dried films.

Hydrophilic/hydrophobic properties of two kinds of NIPs were assessed based on the measurement of pure water contact angles. NIP-PAA and NIP-PMAAA nonporous films were prepared on PMMA slide by dropping the same mixture of monomer solution as porous films. The video contact angle system (VCA-2500 XE, AST products, Billerica, MA) was employed to perform the sessile drop method. At least six stabilized contact angles from different sites of each sample were obtained to calculate average contact 
angle and standard deviation.

\subsubsection{Evaluation of MIPs}

Incubation tests were conducted by weighing approximately $6-10 \mathrm{mg}$ of dried (MIPPMAAA, NIP-PAA and NIP-PMAAA) films in a $20 \mathrm{~mL}$ DNT solution for 2 days to reach adsorption equilibrium. Tests were performed with DNT concentrations of 1, 2.5, 5, 7.5, 10, and 15 ppm. DNT concentrations were determined by high-performance liquid chromatography (HPLC) coupled with a $15-\mathrm{cm}$ by $4.6-\mathrm{mm}$ Epic C18 column. Methane/water $(50: 50, \mathrm{v} / \mathrm{v})$ was used as the mobile phase at flow rate of $0.75 \mathrm{~mL} \cdot \mathrm{min}^{-1}$ with UV Detection at $254 \mathrm{~nm}$ and injection volume of $10 \mu \mathrm{L}$. The fluorescence spectra of MIP films were taken in DI water before and after dipping into the DNT solutions.

Photoluminescent properties were tested on a Hitachi F-4500 Fluorescence Spectrophotometer. Emission scans were performed at an excitation wavelength of 350 $\mathrm{nm}$. The slit width was $5 \mathrm{~nm}$ and scans were conducted in an emission wavelength range of $300 \mathrm{~nm}$ to $700 \mathrm{~nm}$ at a speed of $1200 \mathrm{~nm} \cdot$ minute $^{-1}$. Fluorescence quenching tests were conducted on C-MIPs by DNT solution with concentrations of $1,2.5,5,7.5,10$, and 15 ppm. The time response on fluorescence (FL) intensity of C-MIPs and DNT concentrations change were conducted with initial concentration of $10 \mathrm{ppm}$.

The recognition capacity $(\mathrm{RC})$ is defined as the adsorbate mass per unit of adsorbent mass and is calculated from the equation:

$R C=\left(C_{i}-C_{e}\right) V / m$

Equation 4.2

where $C_{i}$ and $C_{e}$ are the initial and the equilibrium concentrations of the DNT in solution

(in $\mathrm{mg} \mathrm{mL}^{-1}$ ), respectively, $\mathrm{V}$ is the volume of solution (in $\mathrm{mL}$ ) and $\mathrm{m}$ is the mass of the 
films. Then, the imprinting efficiency (IE) is defined by

$I E=R C_{M I P} / R C_{N I P}$

Equation 4.3

where $\mathrm{RC}_{\mathrm{MIP}}$ and $\mathrm{RC}_{\mathrm{NIP}}$ are the recognition capacities of MIP and NIP-PAA, respectively. Selectivity of MIPs

To investigate the competitive recognition ability of MIPs, DNP, 4- NP, and phenol were used as analogues. The mixture of DNT and analogue water solution were prepared at a molar ratio of 2:1 with a concentration of DNT of $0.04 \mathrm{mM}$. Following similar procedures, MIPs were dipped into mixed solution for 30 minutes and FL intensity were measured before and after exposure to DNT and its analogues.

\subsection{Results and Discussions}

\subsubsection{Preparation and Characterization of AC-dots and Porous MIPs}

The TEM image of AC-dots is shown in Figure 4.1 (a) and after background subtraction in Figure 4.1 (b), where the individual nanoparticles can be identified. AC-dots have sizes between $1 \mathrm{~nm}$ to $9 \mathrm{~nm}$ with the average particle size of $5.1 \mathrm{~nm}$, which falls in the range reported in previous studies (Dong et al. 2012a, Zheng et al. 2014b).
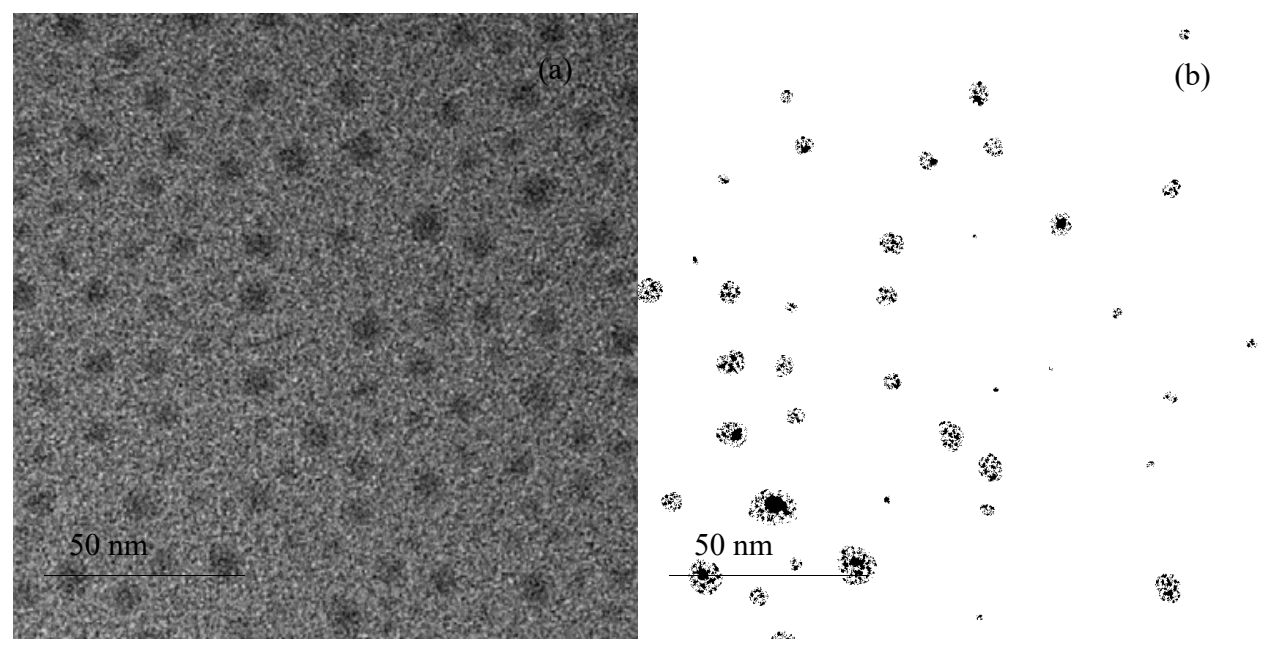

Figure 4.1 TEM image of AC-Dots (a); (b) represents the segmented particles after subtracting the background by ImageJ software 
The surface chemistry of AC-dots was investigated by FTIR. The AC-Dots spectrum showed abundant presence of amino groups on the surface (Figure 4.2). The stretching vibrations of N-H at $3286 \mathrm{~cm}^{-1}$, C-H at $2958 \mathrm{~cm}^{-1}$ and $2819 \mathrm{~cm}^{-1}$ were observed in the high wavenumber region. Two strong peaks at $1658 \mathrm{~cm}^{-1}$ and $1570 \mathrm{~cm}^{-1}$ indicated that the amide linkage was formed. The adsorption bands of $\mathrm{C}=\mathrm{C}, \mathrm{C}=\mathrm{N}, \mathrm{C}=\mathrm{C}-\mathrm{O}$ and epoxy groups were identified at $1466,1362,1300,1111 \mathrm{~cm}^{-1}$, respectively.

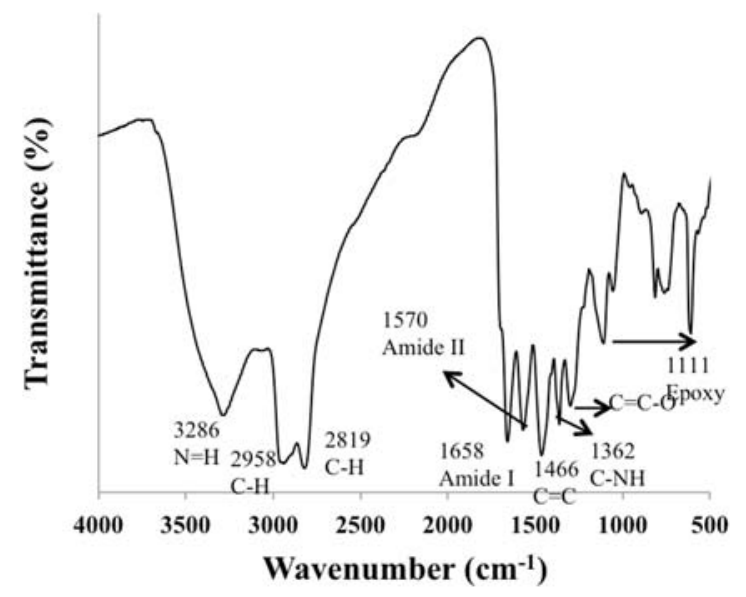

Figure 4.2 FTIR spectrum of AC-dots

Prior to their attachment to the polymeric films, the photoluminescence of AC-dots suspended in aqueous solutions was investigated, as well as the fluorescence quenching by the presence of DNT. AC-dots displayed strong photoluminescent properties and, in the range of $\mathrm{pHs}$ from 3.79 to 9.90 ; the FL intensity increased as $\mathrm{pH}$ decreased (Figure 4.3). With decreasing $\mathrm{pH}$ values, the surface of the AC-Dots became more positively charged (see the zeta potential in Figure 4.4) due to the protonation of amino groups, which is in agreement with previous studies (Wang et al. 2015a). This indicates the surface state plays a key role in FL of AC-dots (Dong et al. 2012b). At low pHs, the positively charged nanoparticles stayed well dispersed due to electrostatic repulsion and 
exhibit higher FL intensity. Therefore, the stability of the AC-dots and their FL activities were enhanced at acidic environment.

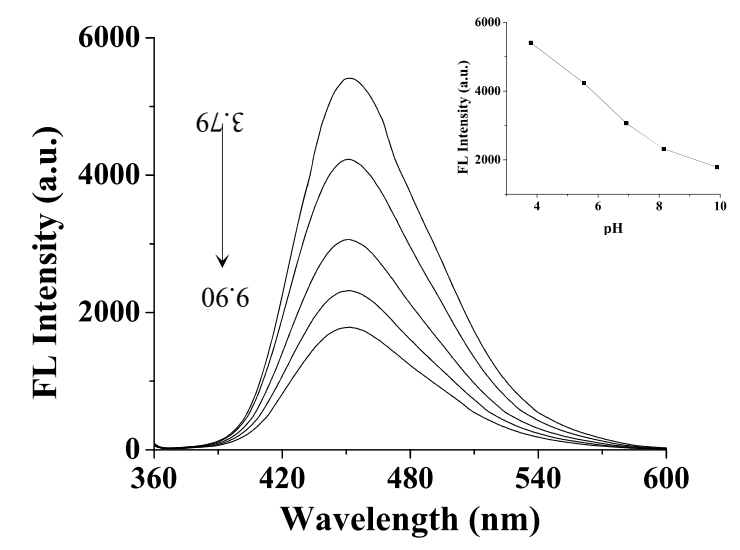

Figure 4.3 Fluorescence spectra of A-CDs at different $\mathrm{pH}$, and inset graph shows plot of FL peak vs. pH

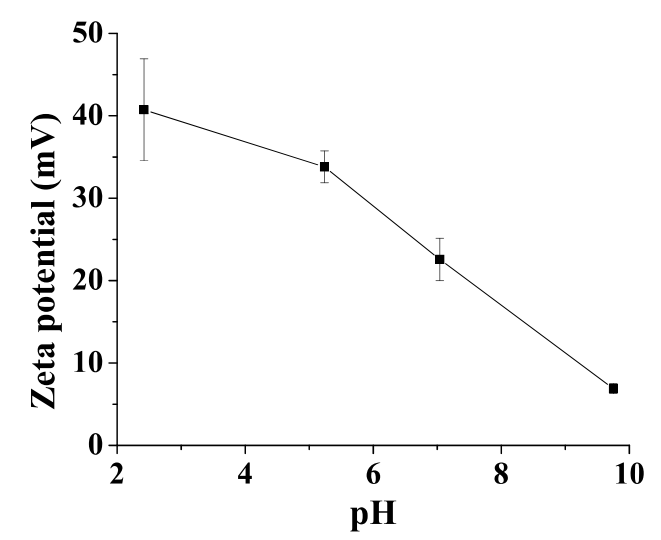

Figure 4.4 Zeta potential of AC-Dots at different $\mathrm{pH}$

The fluorescence quenching tests of AC-dots by DNT demonstrated there was significant interaction between the particles and the contaminant. The FL of AC-dots (Figure 4.5) decreased with increasing concentration of DNT. Static quenching might occur as ACdots formed stable charge-transfer complex with DNT molecule, because of the strong electron-withdrawing ability of nitrated compounds. The FL was reduced since the DNT is essentially reducing the number of AC-dots that can emit. 


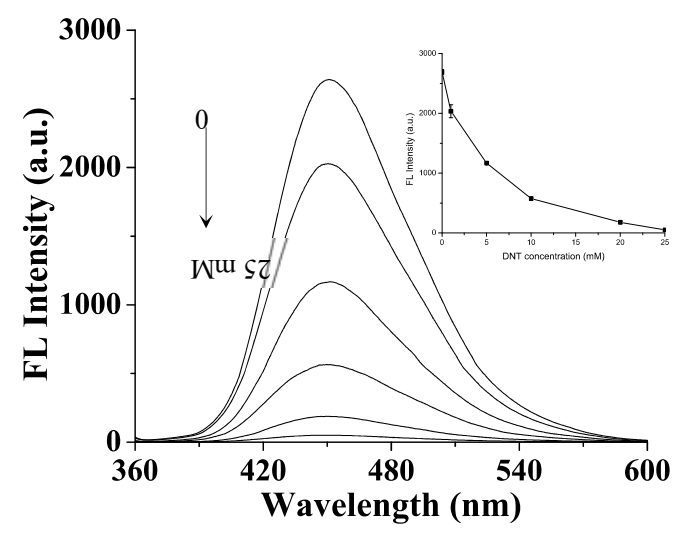

Figure 4.5 Fluorescence spectra of AC-dots with increasing concentrations of DNT, and inset graph shows FL intensity vs. DNT concentrations

Porous structure of MIP films was given by templating with monodispersed silica particles. Silica particles of narrow size distribution were obtained through strict control of the synthesis parameters. SEM images of particle templates are shown in Figure 4.6 (a). The particle sizes obtained by SEM were $213 \pm 9 \mathrm{~nm}$, and $231 \pm 1 \mathrm{~nm}$ when measured by DLS. A micrograph of the porous films is shown in Figure 4.6 (b). This porous macrostructure can provide a high surface area for abundant available binding sites.

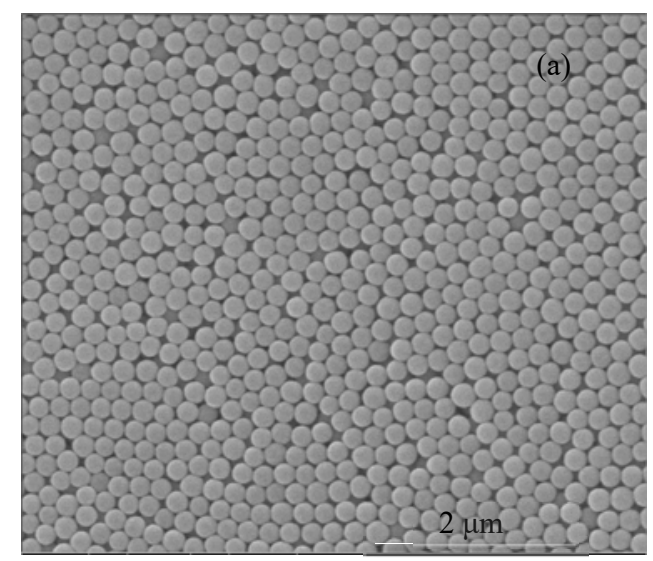



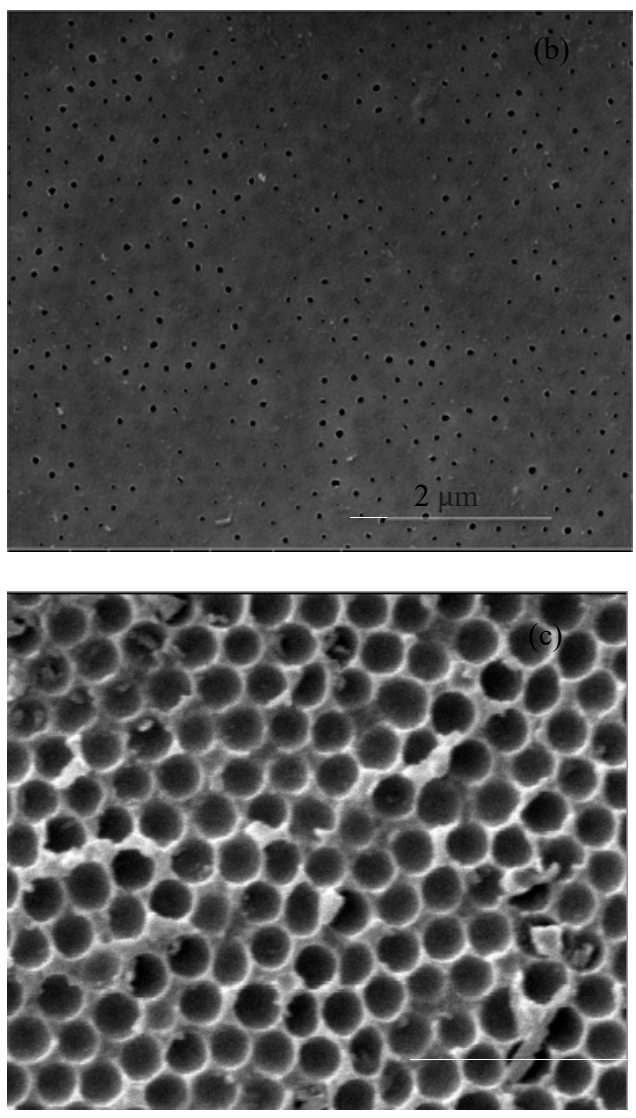

Figure 4.6 SEM images of silica particles (a), porous film surface (b), and inner structure (c).

The significant increase of a steady fluorescence emission of porous films (Figure 4.7) after extensive washing to remove imprinting DNT molecules, strongly suggests the successful formation of covalent bonds between amino groups of AC-Dots and the carboxyl groups of the polymer.

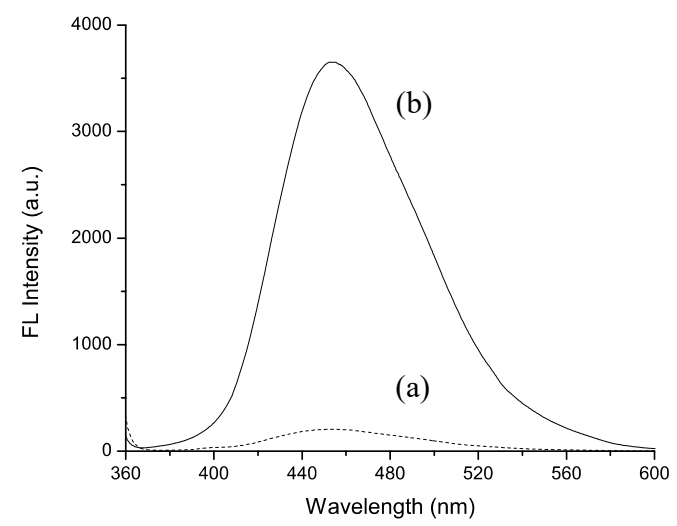


Figure 4.7 Fluorescence spectra of Copolymer (a) and AC-Dots labeled Copolymer (b)

In order to assess whether the fluorescent labeling process may destroy part of the imprinting cavities, the recognition capacity of films prepared by three different approaches were compared: a non-labeled MIP, a MIP subjected to DNT removal and then labeling, and a MIP labeled before DNT removal. The materials were incubated for 30 minutes in a $10 \mathrm{ppm}$ solution of DNT. No significant differences in recognition capacities among the three types of films were observed (Figure 4.8), which proves the fluorescent labeling does not harm the imprinted binding sites.

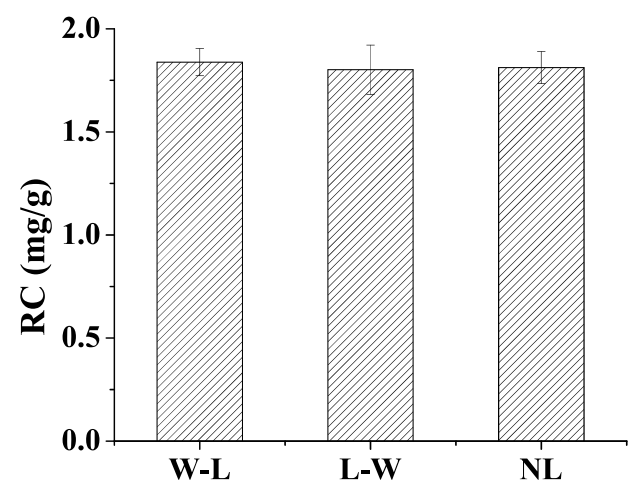

Figure 4.8 Recognition Capacities of label-wash films (L-W), wash-label films (W-L), and non-labeled films (NL) at DNT concentration of $10 \mathrm{ppm}$

\subsubsection{Recognition Capacities of MIPs, NIP-PMAAA, and NIP-PAA}

The recognition capacities of the MIPs, NIP-PMAAA and NIP-PAA were investigated through affinity adsorption experiment. Figure 4.9 shows the binding performance of the MIPs, NIP-PMAAA and NIP-PAA with different concentrations of DNT. For all of the concentrations studied, the MIPs exhibited higher adsorption capacity of DNT than the NIPs, with the IEs in the range of 2.0 to 2.5 (using NIP-PMAAA as control). The higher 
adsorption of MIPs is the result of the combination of specific adsorption of DNT due to the cavities provided by molecularly imprinting and nonspecific adsorption. In contrast, only nonspecific adsorption may occur in the NIP films. In the range of concentrations investigated, both MIPs and NIPs exhibited relatively linear increase of recognition capacity with concentration; however, RC of MIPs changed at a faster rate than NIPs. This denotes a higher affinity of DNT towards the MIPs; the saturation of binding sites of MIPs was not reached in the range of concentrations tested.

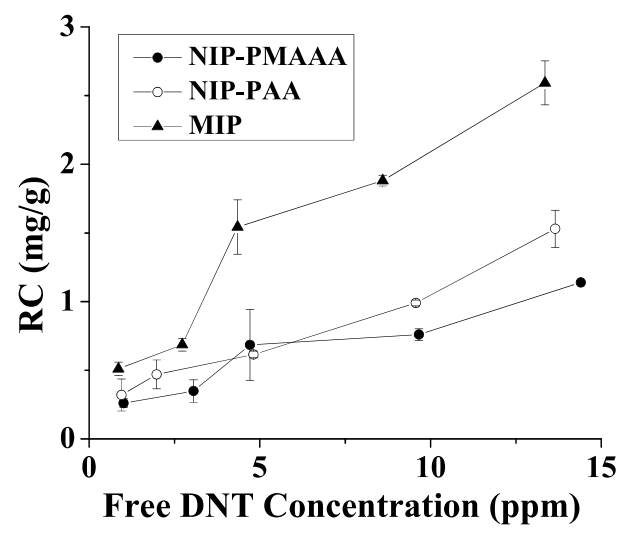

Figure 4.9 Recognition capacities of NIP-PMAAA, NIP-PAA and MIP

DNT is an aromatic nitro compound, which shows great tendency toward reactive and electron-rich radicals, and therefore may act as a retarder in vinyl polymerization, which slows the polymerization of PAA (Mishra and Yagci 2008). For this reason, MA was introduced as a copolymer for the fabrication of MIP films, as opposed to the more hydrophilic AA. The mechanism of radical termination involves attack on both the aromatic ring and the nitro group, but nitro compounds have very little effect on methyl acrylate (Mishra and Yagci 2008). Thus, the presence of a suitable comonomer can minimize the inhibitory effect of DNT on copolymerization. 
PMAAA is more rigid than PAA because the presence of a hydrophobic monomer (MA) improves the mechanical properties (Katime et al. 2000), which in turn may result in slow response due to a decreased water absorption of the film and non-specific interaction with compounds. However, non-specific adsorption by NIP-PAA at most concentrations studied was slightly higher than NIP-PMAAA, although the difference was not significant. The increased adsorption was attributed to the hydrophobic/hydrophilic properties of PAA and PMAAA. PAA films are hydrogels and more hydrophilic than PMAAA as evidenced by the higher swelling ratios of PAA at neutral and basic environments (Figure 4.10) and contact angle measurements performed on nonporous thin films of $64 \pm 2^{\circ}$ for PAA and of $96 \pm 2^{\circ}$ for PMAAA. The presence of MA made the films more manageable with negligible impact on the non-specific adsorption, providing a good balance of hydrophilicity and mechanical strength.

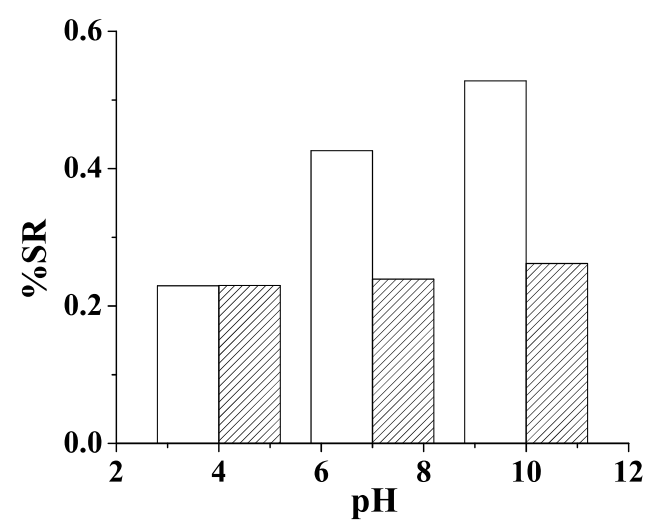

Figure 4.10 Swelling ratio \% of nonporous PAA (open) and PMAAA films (hatched)

DSC is generally used to investigate miscibility in polymer blends or complexes; DSC thermograms (Figure 4.11) showed that both films went through a glass transition process with glass transition temperatures $\left(\mathrm{Tg}_{\mathrm{g}}\right)$ around $50{ }^{\circ} \mathrm{C}$, which stands for the 
temperature at which films changes from a glassy state to a rubbery state. Both NIP-PAA and NIP-PMAAA films showed only a single glass transition temperature, suggesting that the copolymer formed fully miscible complexes, with a homogeneous amorphous phase (Liu et al. 2004).

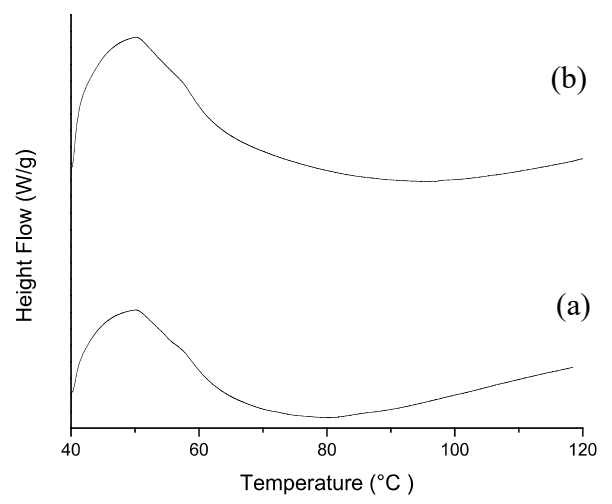

Figure 4.11 DSC thermograms of NIP-Copolymer (a) and NIP-PAA (b)

The chemical structures of PAA and copolymer were investigated by FTIR and ${ }^{1} \mathrm{H}$ NMR spectroscopy. The FTIR spectra were compared in Figure 4.12. The peaks at around $2955 \mathrm{~cm}^{-1}$ for both PAA and copolymer are associated with the methylene $\left(-\mathrm{CH}_{2}-\right)$. The bands due to the carbonyl group $-\mathrm{C}=\mathrm{O}$ of PAA and copolymer overlap at $1737 \mathrm{~cm}^{-1}$. The absence of the peaks at $\sim 1600 \mathrm{~cm}^{-1}$ of unsaturated $\mathrm{C}=\mathrm{C}$ stretch for both samples proves the absence of monomer impurities. The spectrum also displays bands at $1450 \mathrm{~cm}^{-1}$ (scissors of $\mathrm{CH}_{2}$ ), $1230 \mathrm{~cm}^{-1}$ (OH bending of carboxyl group) and $\sim 1170 \mathrm{~cm}^{-1}$ (C-O stretch). 


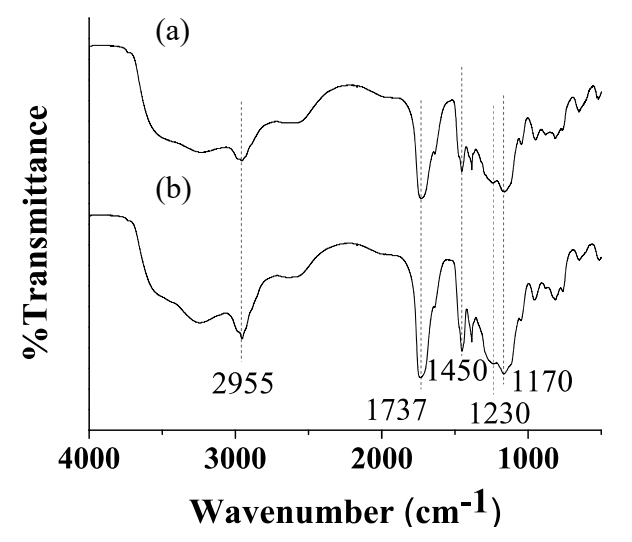

Figure 4.12 FTIR spectra of NIP-PMAAA (a) and NIP-PAA (b)

The presence of the poly methyl acrylate in PMAAA was confirmed by quantitative ${ }^{1} \mathrm{H}$ NMR spectroscopy, which revealed a methyl ester $\left(\mathrm{CH}_{3}\right)$ at $3.75 \mathrm{ppm}$, methylene $\left(\mathrm{CH}_{2}\right)$ at $2.23 \mathrm{ppm}$, and methine $(\mathrm{CH})$ at $2.84 \mathrm{ppm}$. In comparison, the ${ }^{1} \mathrm{H}$ NMR spectrum of PAA does not have corresponding chemical shift, which confirms that poly methyl acrylate does not exist in PAA. 
(A)

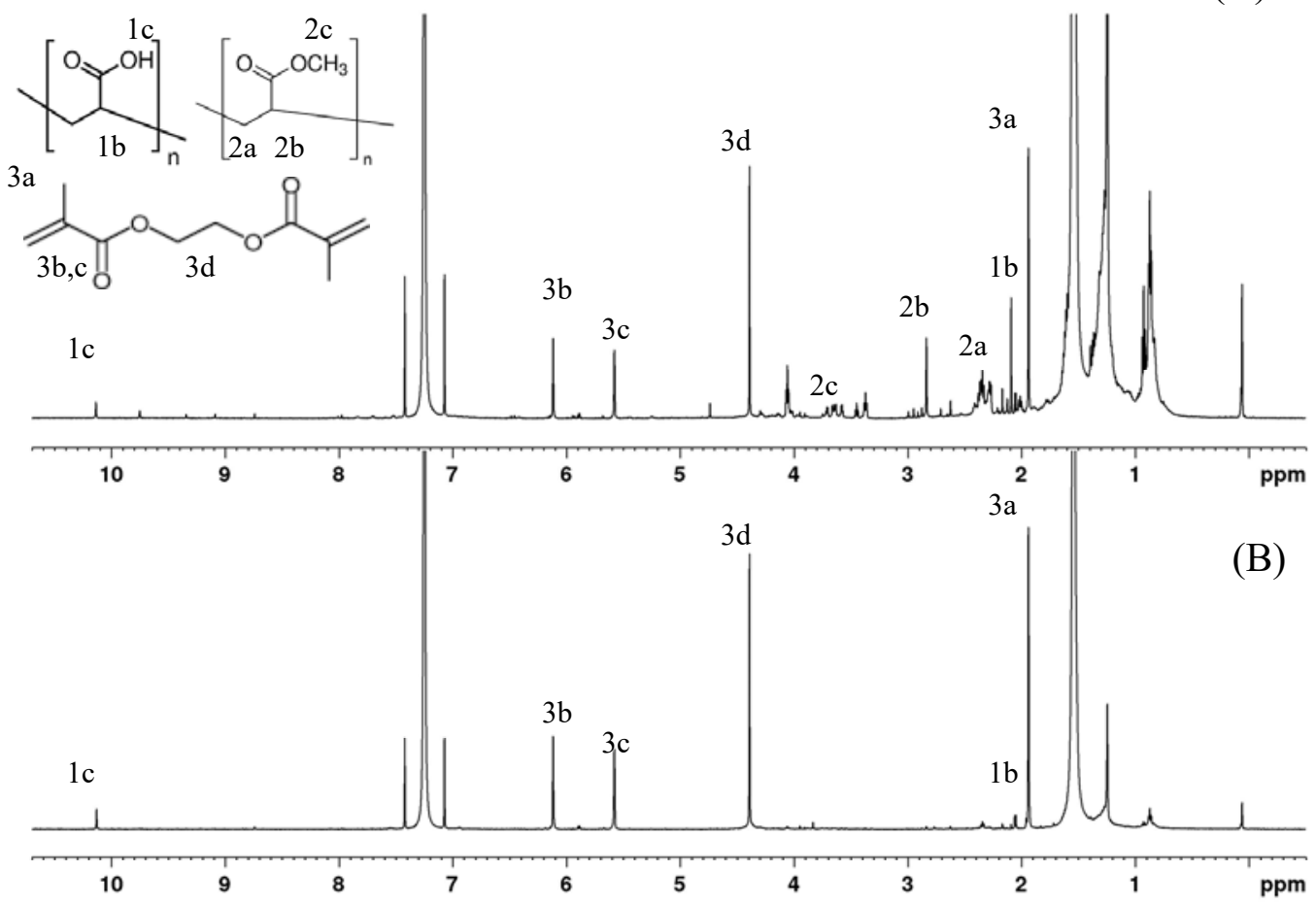

Figure 4.13 ${ }^{1} \mathrm{H}$ NMR spectra of NIP-Copolymer (A) and NIP-PAA (B)

\subsubsection{Photoluminescence Properties of MIP Sensors}

After adsorption tests, the change in fluorescence intensity of MIP films with DNT concentration was measured. The fluorescence intensity of the MIP film was quenched gradually with increasing concentration of DNT (Figure 4.14). Generally, the fluorescence quenching depends on the adsorptive affinity of the films and DNT. As the FL intensity of films was measured after taken out from the DNT solution and rinsing with clean water, quenching by nonspecific adsorption was diminished. The methodology minimizes interferences by other solution constituents. Hence, the sensor provides 
specific recognition for DNT rather than general quenchers. The fluorescence quenching in this system followed the Stern-Volmer equation (Wahba et al. 2015).

$F_{o} / F=1+K_{s v} C_{q}$

Equation 4.4

where $\mathrm{F}_{0}$ and $\mathrm{F}$ are the fluorescence intensity in the absence and presence of quencher, respectively, $\mathrm{C}_{\mathrm{q}}$ is the concentration of the quencher, and $\mathrm{K}_{\mathrm{sv}}$ is the quenching constant for the quencher.

The analytical performance of the MIPs was evaluated by the Stern-Volmer plot. It showed a linear relationship between $\mathrm{F}_{0} / \mathrm{F}$ and DNT concentrations from $1 \mathrm{ppm}$ to 15 ppm (Figure 6 b) with a correlation coefficient of 0.998 . The detection limit was calculated to be $0.28 \mathrm{ppm}(\mathrm{S} / \mathrm{N}=3)$. A golden standard for detection of aqueous DNT can be reached to a few ppb by chromatographic method in combination with various detectors (2014). The detection limit of our sensor differs two orders of magnitude. However, our sensor is at low cost, easily fabricated and operated and it could be a good alternative for on-site detection of DNT contaminated field.

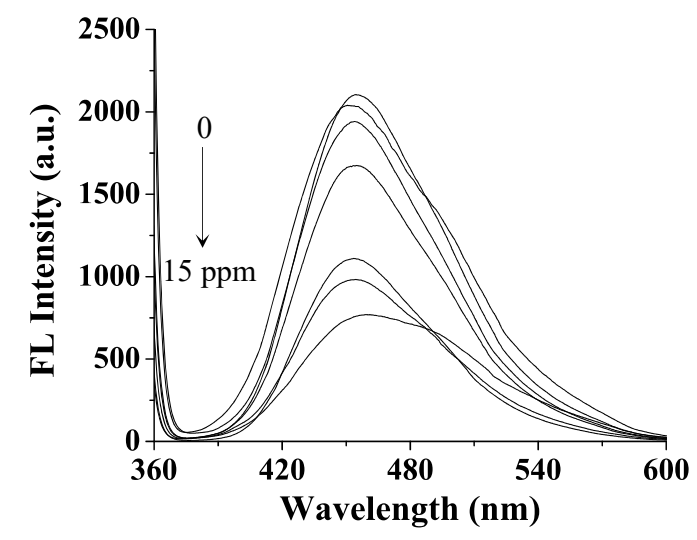




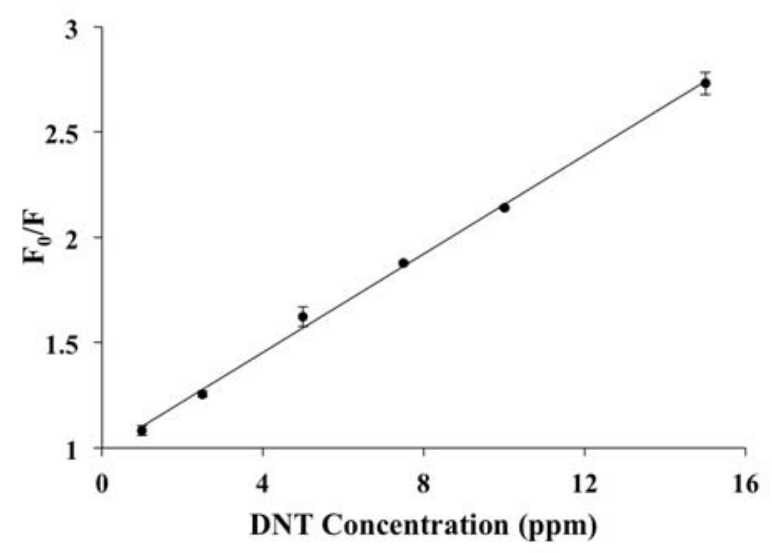

Figure 4.14 Fluorescence emission spectra of C-MIPs with an increasing concentration of DNT (a) and Stern-Volmer plot of C-MIPs with an increasing concentration of DNT (b)

The response time of the fluorescence intensity for DNT was studied. A MIP film was place in a $10 \mathrm{ppm}$ DNT solution and removed periodically to measured FL intensity; additionally, the test solution was sampled over time to quantify the remaining amount of DNT in solution. The sensor had a fast response to the presence of DNT. Figure 4.15 shows the fluorescence intensity decreased rapidly in the first 10 minutes, followed by a slow decrease with minimal changes up to 30 minutes. The adsorption kinetics of DNT on the films was also measured; the remaining concentration in the liquid showed a trend similar to the FL response (Figure 4.15). These results further support the concept that the FL intensity decrease was due to the adsorption in the films, as it was fast during the initial 10 minutes and slower after that, almost reaching equilibrium after approximately 30 minutes. 


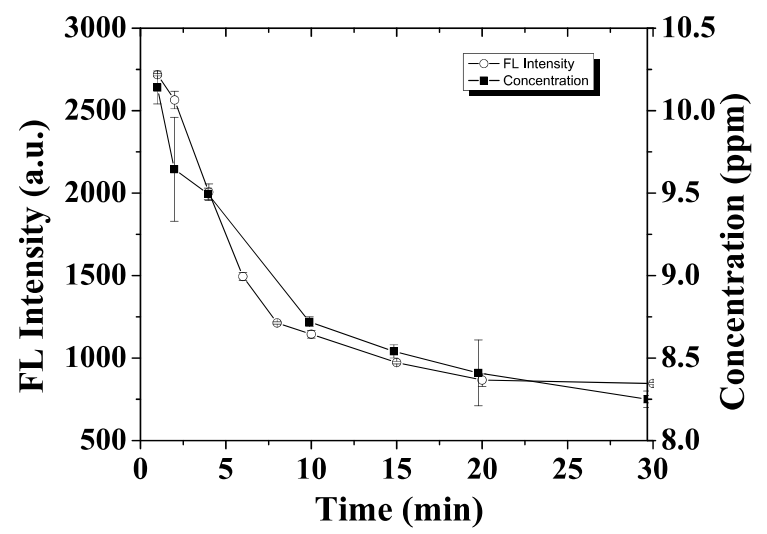

Figure 4.15 Fluorescence response time of C-MIP for DNT and adsorption kinetics of MIP with initial concentration of $10 \mathrm{ppm}$

The economic feasibility of the sensor as well as its practicality is strongly related to the ability to recycle the films after each use. Theoretically, the same approach employed to remove the target molecule after fabrication would result in successful regeneration. However, polymeric structures may suffer from relaxation of their structures after successive use with the consequent loss of recognition capacity due to deformation of binding cavities. Additionally, molecules may adsorb non-specifically and irreversibly on the C-dots, preventing the recovery of initial PL levels. We investigated the reusability of the sensor conducting 5 cycles of DNT removal and incubation for 30 minutes in $10 \mathrm{ppm}$ DNT solutions. The results revealed that the fluorescence labeled sensor had good reusability, with a slightly decrease of 12\% for initial FL intensity (Figure 4.16). 


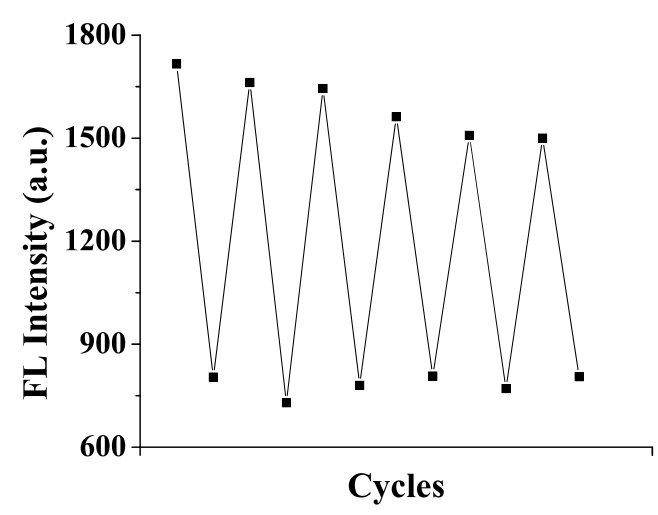

Figure 4.16 The cycle curves of the AC-Dots labeled MIPs in the absence (the upper dots) and the presence (the bottom dots) of $10 \mathrm{ppm}$ DNT

\subsubsection{Selectivity of MIP sensors}

The competitive binding tests were conducted on DNT and its mixture with phenol, 4-NP, and DNP, respectively. The ratio of the net decline in emission to initial emission intensity of the sensor, calculated as $\left(\mathrm{F}_{0}-\mathrm{F}\right) / \mathrm{F}_{0}$, was used as the index of quenching. The quenching amount by DNT and phenol was slightly lower than the control, while it was somewhat higher for the mixtures of DNT with 4-NP and DNP. By using the correlation obtained by Stern-Volmer plot, the apparent concentrations of DNT in the presence of its analogues were calculated.

Compared with the same concentration $(7.7 \mathrm{ppm})$ pure DNT solution, the apparent concentration of $0.04 \mathrm{mM}$ DNT with $0.02 \mathrm{mM}$ phenol was $6.8 \mathrm{ppm}$, which was about $12 \%$ decrease than the real concentration of DNT. Phenol molecules can compete with DNT for the binding sites, given their similar structure, but lack the ability to quench the ACdots FL and therefore its presence resulted in underestimation of the DNT concentration. For DNT in combination with 4-NP and DNP, the sensor estimated concentrations of 
DNT were 8.4 ppm and 9.4 ppm, respectively. Both compounds may compete for binding sites and produce quenching, as 4-NP and DNP both have nitro groups; DNP is expected to show more electron withdrawing ability and a superior quenching effect as it has twice as many of nitro groups per mole than 4-NP. Moreover, DNP is closer in size and shape to DNT, and thus the chance to be recognized by the imprinting cavities is higher than 4NP, resulting in a higher calculated concentration of DNT. However, the readings of DNT concentration in presence of analogues were $9 \%$ and $22 \%$ increase, respectively. In comparison with the very challenging 50\% dosage of analogues, the errors induced were low, and it can be concluded that the sensor exhibited good selectivity to its target molecule.

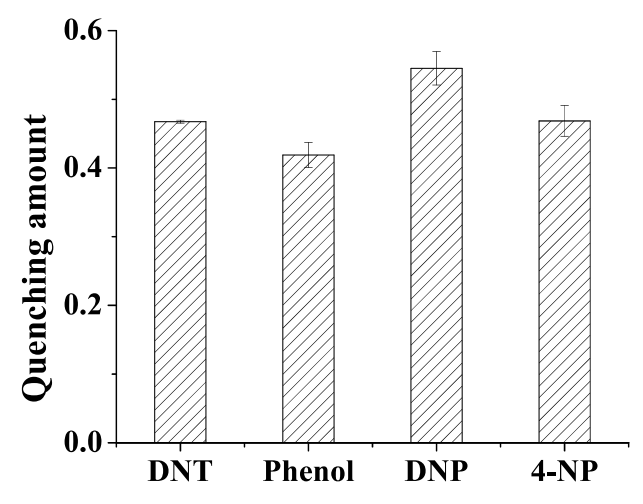

Figure 4.17 Quenching amount of DNT in presence of analogues; [DNT] $=0.04 \mathrm{mM}$; [analogues] $=0.02$ $\mathrm{mM}$.

\subsection{Conclusions}

AC-dots labeled DNT-MIPs were developed for selective detection of DNT based on the combination of molecularly imprinted polymers and fluorescence quenching. The sensors had a high surface area provided by the macroporous structures for more easily available binding sites. Moreover, it displayed higher recognition capacities of DNT than NIPs at 
all concentrations investigated (IEs 2-2.5) because of the specific adsorption of the target. On the other hand, this fluorescence sensor showed a rapid response and was able to provide quantitative measurements in the linear range between 1 to $15 \mathrm{ppm}$ with a detection limit of $0.28 \mathrm{ppm}$. Selectivity tests further proved the good recognition of target molecule of MIPs in the presence of potential interferences (structural analogues), with reasonably good estimates of DNT concentrations.

The AC-dots labeled MIP sensors have overcome the environmental toxicity of Q-dots based sensors in terms of leaching of heavy metals. In addition, compared with previously reported MIP capped C-dots systems (Hao et al. 2016, Liu et al. 2016, Wang et al. 2015b), this films are easier to operate and can better distinguish the target contaminant interferences because the sensor can be rinsed after capturing and before FL measurement, which provides an opportunity for the removal of loosely bound, nonspecifically adsorbed compounds. Moreover, the material can be recovered after measurement and regenerated for continuous use, lowering costs and providing a noninvasive method of analysis (no nanomaterials are left in the sample). It shows a good reusability till 5 cycles. However, DNT concentration reading of the sensor may be affected by the presence of interferences (heavy metal such as copper, natural organic matter, and structural analogues). Further research will be conducted to explore its applicability for real water sample.

In conclusion, the AC-dots labeled DNT-MIP films exhibit good selectivity and sensitivity with the ability to quantitatively analyze the concentration of DNT. The system has the potential to be further developed for a platform of fast, easy and on-site detection for DNT contamination. 


\section{Chapter 5. Effect of $\mathbf{p H}$, ionic strength and natural organic matter on the DNT-MIP fluorescent sensor}

Molecular Imprinted Polymer (MIP) based sensors had been successfully applied to the quantitative detection of specific contaminants. In order to obtain a useful sensor device, different transducer mechanisms have been combined with MIPs, such as optical, chemical or electrical properties variations. Among them, the optical MIP-based molecular recognition sensors have been developed due to the covalent linkage of MIPs to optical transducers. The MIP binding efficiency, recognition processes and selectivity have been intensively studied by optical means due to the general high sensitivity and simplicity of the utilization of optical techniques (Henry et al. 2005). The sensing properties can also be characterized by parameters such as linearity, limit of detection, sensitivity, selectivity, reproducibility, accuracy, robustness, response time and lifetime (Li et al. 2012). However, the effects of sample characteristics commonly encountered in natural waters (i.e. variations of $\mathrm{pH}$, ionic strength, natural organic matter content) on the sensing performance of optical MIPs are not fully understood. MIPs have been reported which respond to stimuli such as change in temperature (Pan et al. 2013), pH (Kanekiyo et al. 2003), and ionic strength ( $\mathrm{Wu}$ et al. 2010). Interferences are another concern because they can affect the recognition of MIPs. In the context of this study, we define interference as a compound that will hinder the access to capture sites of the target molecule by non-specific adsorption onto the polymer surface resulting in pore blocking, steric repulsion or electrostatic interactions at or near the interface, and produce a decrease in the capacity of the MIP. Potential interferences in natural waters are dissolved organic matter, dissolved salts, inorganic colloidal particles, and microorganisms. Given 
the fact that most efforts have been devoted to the synthesis of MIPs as SPE media, research in this area has been directed mostly towards the conditioning, washing and eluting stages of the process rather than the capture and sensing, and much of the actual mechanisms by which this interference may occur are not fully understood. The potential for MIP-based sensors to be used in-situ is promising if the influences of these parameters are explored and interpreted.

Some research has been reported as the use of MIPs as the sorbent for solid-phase extraction of natural water samples (Davoodi et al. 2014, Kueseng et al. 2009). The direct use of MIPs and MIP-enabled devices as sensors in natural waters has not been fully explored (Alizadeh et al. 2010, Hao et al. 2016), especially regarding the effects of the different parameters in the water chemistry matrix. Conductometric transducer based atrazine sensor allowed the monitoring of atrazine both in contaminated and uncontaminated natural waters as the detection limit was $1 \mathrm{ppb}$. The only draw back was that it required laboratory based instrumentation and was not suitable for field studies (Rao et al. 2007). Atrazine and its analogs simazine, ametryn and propazine could be successfully extracted from natural water samples, using atrazine as the template molecule (Wen et al. 2012). In another study, diphenolic Acid (DPA) and bisphenol A (BPA) were used as dummy template molecules for the capture of tetrabromobisphenol A (TBBPA). The reported recoveries of TBBPA in tap, river and lake water ranged from $85 \%$ to $97 \%$ with detection limit as low as $2 \mathrm{ng} / \mathrm{mL}$ (Yin et al. 2012).

Fluorescence based sensors are particular suited for field use, as they are generally sturdy and easy to measure. In this area, fluorescent nanoparticles are preferable to fluorescent polymers, as they have shown to be brighter and more stable; however, leakage of the 
nanomaterial into the sample may be a concern, if the particles are not strongly bonded to the MIP matrix. Sample chemical matrix may produce additional interference by interacting or preventing interaction between the target compound and the fluorescent material, leading to over or underestimations of the true concentration of the target. Detection of contaminants in natural waters at environmentally relevant concentrations is particularly challenging, due to the complex and potential variability of its chemical composition.

We recently reported a novel C-dot fluorescent label MIP based sensor for the detection of 2,4-dinitrotoluene (DNT) in water samples in a range from 1 to $15 \mathrm{ppm}$, that allows for quantitation of DNT in aqueous solutions, with a detection limit of $0.28 \mathrm{ppm}$. A thin porous film was synthesized by templating with monodispersed silica particles. The porous macrostructure provided a high surface area for abundant and easily available binding sites. A strong, covalent bonding between AC-dots and polymer was created by linking amino groups and carboxyl groups with N-hydroxysuccinimide (NHS) and N-(3Dimethylaminopropyl)-N'-ethylcarbodiimide hydrochloride (EDC) and provided superior durability, avoiding the loss of nanoparticles and ensuring fluorescence stability (Dai et al. 2017). In this study, we investigated the field application of the MIP sensor labeled with fluorescent nanoparticles (carbon dots) for the detection and quantification DNT in natural water conditions. The effect of salinity, $\mathrm{pH}$ and dissolved organic matter on the sensor performance was analyzed. Two factors may potentially play important roles: interference with the nonspecific and specific adsorption of the DNT onto the MIP, and additional quenching by solutes other than the DNT. A methodology was established to minimize those effects and/or create an adjusted calibration for determination of DNT 
concentrations under different background water matrixes.

The MIP based sensors can be successfully used for quantitative detection of specific contaminants. The potential to be used in-situ is very promising if the effects of characteristics (i.e. $\mathrm{pH}$, ionic strength, natural organic matter) in natural water are explored and understood. A variety of MIPs have been reported which respond to stimuli such as change in temperature (Pan et al. 2013), $\mathrm{pH}$ (Kanekiyo et al. 2003), and ionic strength (Wu et al. 2010).

Our objectives are to investigate the applicability of our sensors in various aqueous environments and try to understand the role of natural organic matter and water chemistry on the effect of DNT rebinding and carbon dots fluorescence quenching.

\subsection{Materials and Method}

\subsubsection{Materials}

Chemicals were purchased from Sigma-Aldrich (https://www.sigmaaldrich.com/unitedstates.html) and used without further purification; tetraethoxysilane (TEOS) (98\%), ammonia solution ( $25 \%$ in water), ethanol (200 proof, ACS reagent, $99.5 \%)$, acrylic acid (AA) (99\%), methyl acrylate (MA) (99\%), ethylene glycol dimethacrylate (EGDMA) (98\%), 2,2'-azobisisobutyronitrile (AIBN) (98\%), 2,4-dinitrotoluene (DNT) (97\%), phenol, 4-nitrophenol (4-NP) (99\%), and dinitrophenol (DNP) (98\%), hydrofluoric acid (HF) (48\%), citric acid (CA) (99.5\%), branched polyethylenimine (BPEI), Nhydroxysuccinimide (NHS), and N-(3-Dimethylaminopropyl)-N'-ethylcarbodiimide hydrochloride (EDC).

$\mathrm{NaOH}$ and $\mathrm{HCl}$ (Fisher Scientific) were used for $\mathrm{pH}$ adjustment; ionic strength of the 
samples was controlled by addition of $\mathrm{NaCl}$ (reagent grade, Acros Organics) or $\mathrm{CaCl} 2$ (reagent grade, J.T.Baker). Suwannee River NOM (SRNOM) was used as a model of natural organic matter and purchased from International Humic Substances Society (St. Paul, MN, USA).

\subsubsection{Synthesis of MIPs and Fluorescent labeling}

The fluorescence labeled MIP films were prepared as described in our previous work (Dai et al. 2017). Non-imprinted polymer films (NIP) films were synthesized following a similar procedure without adding DNT and played as control for the MIP sensors in the tests. The porosity of the MIP films was created using sacrificial colloidal crystals made of silica particles (200 $\mathrm{nm}$ diameter) as templates followed by etching of the particles. Monodisperse silica particles were synthesized following the Stöber method (Stöber et al. $1968)$ by the reaction of TEOS $(8.3 \mathrm{~mL})$, ethanol $(200 \mathrm{~mL})$ and ammonia $(18.3 \mathrm{~mL})$ The particles were centrifuged and dispersed twice in DI water and once in ethanol for purification. Colloidal crystals were fabricated by vertical self-assembly on a glass substrate as described elsewhere (Jiang et al. 1999). In this procedure, a cleaned glass slide was vertically placed into a flask containing a $0.5 \%$ volume fraction suspension of silica particles in ethanol. After the volatilization of ethanol, the colloidal crystals were formed on the slide. Another slide was placed upon the colloidal crystal and the two slides were firmly held together. Polymerization solutions were prepared mixing $0.4 \mathrm{~mL}$ (4.4 mmol) AA and $0.4 \mathrm{~mL}(5.8 \mathrm{mmol}) \mathrm{MA}$ as the functional monomer (with the molar ratio of 3:4), $0.55 \mathrm{~mL}$ (2.9 mmol) EGDMA as the crosslinking agent, $6 \mathrm{mg}(0.04 \mathrm{mmol})$ AIBN as the initiator, $4 \mathrm{mg}(0.02 \mathrm{mmol}) \mathrm{DNT}$ as the target molecule and $0.6 \mathrm{~mL}$ ethanol as the solvent, to yield MIP-PMAAA. One end of glass assembly was put in contact with 
the polymerization mixture that rose by capillary forces filling the void spaces within the colloidal crystal. Polymerization was performed under UV light at wavelength of $365 \mathrm{~nm}$ for $5 \mathrm{~h}$ at room temperature. Silica particles were removed by submerging in $5 \%$ hydrofluoric acid bath. Finally, the films were soaked in ethanol in order to elute DNT, changing the solvent every half an hour for $3 \mathrm{~h}$.

Amino functionalized carbon dots (AC-dots) were synthesized following a published method (Zheng et al. 2014b). The porous MIP films were placed into a $0.033 \mathrm{mM}$ EDC / $0.033 \mathrm{mM}$ NHS aqueous solution. After stirring for $1 \mathrm{~h}$ at room temperature, $2 \mathrm{~mL}$ solution of AC-dots with a concentration of $5 \mathrm{~g} \cdot \mathrm{L}^{-1}$ was added dropwise. The mixture was stirred for $36 \mathrm{~h}$ at room temperature in the dark to form the covalent bonding between the AC-dots and the films.

\subsubsection{Characterization}

Silica particles and porous films were imaged by a FEI Quanta 600 FEG Environmental Scanning Electron Microscopy (ESEM) to investigate the pore size and morphology. Samples were attached to a metal mount using carbon tape and were coated with a thin layer of platinum to provide a conductive surface using a sputter coater (Emitech K575x). Fourier Transformed Infrared Spectroscopy (FTIR) spectrum of AC-dots was collected with a Nicolet 4700 FT-IR in order to identify the functional groups present on the surface of AC-dots.

Swelling experiments of NIP-PMAAA were performed in: (1) phosphate buffer at three different $\mathrm{pH}$ values (4, 7 and 10) at room temperature; and (2) $\mathrm{NaCl}$ or $\mathrm{CaCl}_{2}$ solution at different ionic strengths $(1,10$ and $100 \mathrm{mM})$ at room temperature. The polymers were 
swollen in solutions for $48 \mathrm{~h}$ at ambient temperature and the degree of swelling was determined gravimetrically. The percentage swelling ratio (\%SR) was calculated from the expression 5.1.

$\% \mathrm{SR}=\left(\mathrm{m}_{\mathrm{s}}-\mathrm{m}_{\mathrm{d}}\right) / \mathrm{m}_{\mathrm{d}}$

Equation 5.1

where $\mathrm{ms}$ is the mass of the swollen film at equilibrium and md is the mass of the freeze dried films.

\subsubsection{Fluorescence Quenching Experiments}

Photoluminescent properties were investigated on a Hitachi F-4500 Fluorescence Spectrophotometer. Emission scans were performed at an excitation wavelength of 350 $\mathrm{nm}$. The slit width was $5 \mathrm{~nm}$ and scans were conducted in an emission wavelength range of $300 \mathrm{~nm}$ to $700 \mathrm{~nm}$ at a speed of $1200 \mathrm{~nm} \cdot$ minute $^{-1}$.

The initial FL intensity of MIP and NIP films was measured in DI. Secondly, sensor strips were dipped in the corresponding test solutions and incubated for 30 minutes, as determined in previous research13. Then, sensors were rinsed with DI water and their FL intensities were measured submerged in pure water at neutral $\mathrm{pH}$. The level of quenching is calculated as the ratio of the initial intensity to the intensity measured after exposure to the sample, $\mathrm{F}_{0} / \mathrm{F}$. The linear relationship between $\mathrm{F}_{0} / \mathrm{F}$ and $\mathrm{DNT}$ concentration in solution followed Stern-Volmer equation (Wahba et al. 2015).

$\mathrm{F}_{\mathrm{o}} / \mathrm{F}=1+\mathrm{K}_{\mathrm{sv}} \mathrm{C}_{\mathrm{q}}$

Equation 5.2

where $\mathrm{F}_{0}$ and $\mathrm{F}$ are the fluorescence intensity in the absence and presence of quencher, respectively, $\mathrm{Cq}$ is the concentration of the quencher, and $\mathrm{Ksv}$ is the quenching constant for the quencher. 
The effect of $\mathrm{pH}$ on the detection and quantification of DNT by the MIP sensor was investigated in 10 ppm DNT solutions adjusted at three levels of $\mathrm{pH}(4,6,9)$, adding $\mathrm{HCl}$ $(10 \mathrm{mM})$ or $\mathrm{NaOH}(10 \mathrm{mM})$ solutions as needed. The DNT concentration was chosen because it falls into the linear range of the sensor detection range and causes a significant FL decrease. For comparison, the responses of MIPs and AC-Dots $(0.2 \mathrm{mg} / \mathrm{L})$ to $\mathrm{pH}$, both having the similar FL intensities at neutral $\mathrm{pH}$, were tested in absence of DNT as well. The IS effect was investigated in $10 \mathrm{ppm}$ DNT test solutions with a background concentration of at 1,10 , and $100 \mathrm{mM} \mathrm{NaCl}$ or $\mathrm{CaCl}_{2}$.

Organic materials are ubiquitous in natural waters, originating from the decay of plans, animals or anthropogenic pollution. Natural organic matter (NOM) includes substances that can emit or quench fluorescence, as well as adsorb onto the sensor surfaces, and therefore induce error in sensor output. In order to assess this effect, experiments were performed with NIP and MIP sensors in mixture solutions of $10 \mathrm{ppm}$ DNT with different levels of Suwannee River NOM (SRNOM), as a model of natural organic matter, at concentration of 1, 10 and $50 \mathrm{ppm}$. Control tests performed with NIP and MIP sensors at the same SRNOM concentration levels in the absence of DNT.

Finally, the sensor was tested in real water samples collected from Stephens Lake, Columbia, Missouri, and tap water at the University of Missouri (coming from Water Treatment Plant with Columbia Utilities) that were spiked with DNT. Immediately after collection, the sampled water was characterized by $\mathrm{pH}$, conductivity and total organic carbon (TOC) measurements using a Shimadzu TOC-VCPN analyzer.

The MIP-sensor was dipped in the spiked solutions, let to equilibrate for 30 minutes and rinsed with DI before reading fluorescence intensity. DNT concentrations were 
determined by the quenching amount as established by the Stern-Volmer equation. Sensor measurements were validated by analytical determinations of the DNT concentrations in the samples, by high-performance liquid chromatography (HPLC) coupled with a $15-\mathrm{cm}$ by $4.6-\mathrm{mm}$ Epic C18 column. Methane/water (50:50, v/v) was used as the mobile phase at flow rate of $0.75 \mathrm{~mL} \cdot \mathrm{min}^{-1}$ with UV Detection at $254 \mathrm{~nm}$ and injection volume of $10 \mu \mathrm{L}$.

\subsection{Results and Discussion}

\subsubsection{Characterization Results}

SEM

The porous structure of MIP films was given by templating with monodispersed silica particles. Silica particles of narrow size distribution were obtained through strict control of the synthesis parameters. SEM images of particle templates are shown in Figure S1 (a). The particle sizes obtained by SEM were $213 \pm 9 \mathrm{~nm}$, and $231 \pm 1 \mathrm{~nm}$ when measured by DLS. A micrograph of the porous films is shown in Figure S1 (b) and (c). This porous macrostructure can provide a high surface area for abundant and easily available binding sites.

FTIR

The surface chemistry of AC-dots was investigated by FTIR. The AC-Dots spectrum showed abundant presence of amino groups on the surface (Figure S2). The stretching vibrations of $\mathrm{N}-\mathrm{H}$ at $3286 \mathrm{~cm}^{-1}, \mathrm{C}-\mathrm{H}$ at $2958 \mathrm{~cm}^{-1}$ and $2819 \mathrm{~cm}^{-1}$ were observed in the high wavenumber region. Two strong peaks at $1658 \mathrm{~cm}^{-1}$ and $1570 \mathrm{~cm}^{-1}$ indicated that the amide linkage was formed. The adsorption bands of $\mathrm{C}=\mathrm{C}, \mathrm{C}=\mathrm{N}, \mathrm{C}=\mathrm{C}-\mathrm{O}$ and epoxy 
groups were identified at $1466,1362,1300,1111 \mathrm{~cm}^{-1}$, respectively. The surface basic groups resulted in a positively charged surface due to protonation of amino groups. The amino groups can be further protonated with decreasing pHs (Bayati et al. 2017). The quenching ability may be hindered when amino groups are well protonated and thus unable to complex with quenchers (Dong et al. 2012a).

Swelling properties

The equilibrium swelling degree of hydrogels depends on the cross-linking and charge densities of the polymer network. Polyacrylic acid can shrink or swell in response to changes in temperature, $\mathrm{pH}$, ionic strength, solvent quality, electronic field, etc (Okay 2009). The \%SR increased from 4 to 7 and to 10, but not dramatically (Figure 5.1). Unlike pure acrylic acid systems, the swelling properties of hydrogels are less $\mathrm{pH}$ dependent (Chen et al. 2015, Dai et al. 2017). There was still slightly higher nonspecific adsorption at $\mathrm{pH}$ of 9 with higher swelling capacity, which might result from faster response by increased water absorption of the film and non-specific interaction with compounds. For the sensors, the responses may cause more quenching due to higher nonspecific adsorption, especially if NOM is the adsorbate.

The $\%$ SR decreased with increasing concentrations of electrolytes, and the trends were similar for both $\mathrm{NaCl}$ and $\mathrm{CaCl} 2$ (Figure 5.2). Similarly, this swelling behavior may lead to less quenching in the presence of salts due to lower adsorption. 


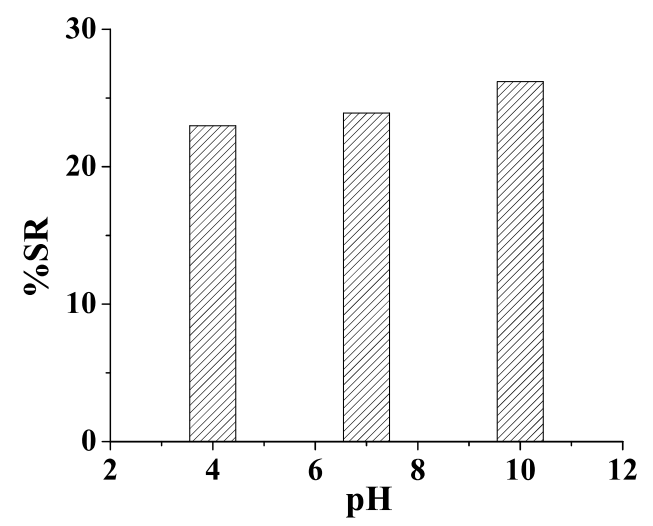

Figure 5.1 Swelling ratios of PMAAA films

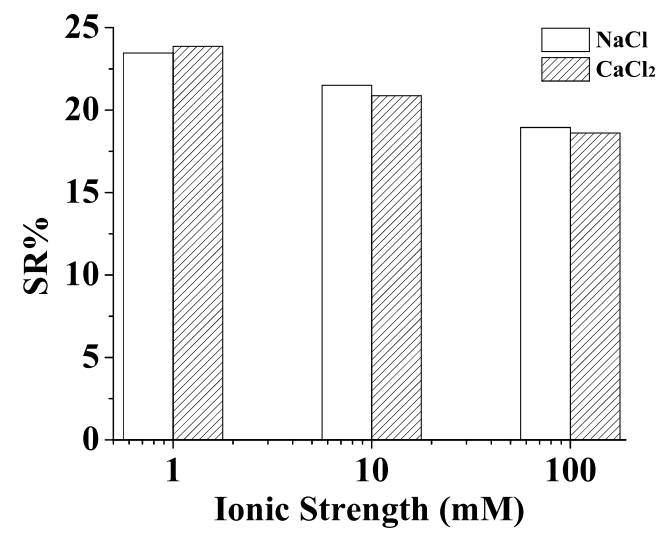

Figure 5.2. Swelling Ratio\% of MIP films at different ionic strength with different salts

\subsubsection{Effect of $\mathrm{pH}$}

The $\mathrm{pH}$ of the sample to be analyzed can affect the operation of the sensor by altering the surface chemistry of the carbon dots and, as a consequence their fluorescent emission, and / or by altering the conformation of the polymer matrix, resulting in deformation of the cavities available for capture. The emission intensity is $\mathrm{pH}$ dependent. AC-dots $(0.2 \mathrm{mg} / \mathrm{L}$ in suspension) exhibited the highest FL intensity at $\mathrm{pH}$ of 4 , and it decreased with increasing $\mathrm{pH}$ (Figure 5.3). Their FL activities was strong at weakly acidic media 
( $\mathrm{pH} 4-6$ ), but weak at other $\mathrm{pHs,} \mathrm{especially} \mathrm{at} \mathrm{pH}>10$. This observation is in agreement with previous research (Dong et al. 2012a), and suggests that the surface states of ACdots play key roles determining their FL activities (Ding et al. 2014).

The tests on effects of $\mathrm{pHs}$ were conducted with initial FL intensities $\left(\mathrm{F}_{0}\right)$ measured in DI water. Then, the MIP-sensors were dipped in acidic, neutral, or basic 10 ppm DNT solutions, and rinsed with DI water before measuring the FL intensities (F), again in clean water. Although the sensor initially showed different responses for the three $\mathrm{pH}$ levels, those were effectively minimized by the rinsing step. Figure $\mathbf{5 . 4}$ shows that, in the absence of DNT, exposing the MIP films to acid or base solutions, the fluorescence intensity changed accordingly. However, after the clean water wash, the effects are less evident and $\mathrm{F}_{0} / \mathrm{F}$ much closer to 1 as expected since no DNT molecules were present. This represents a significant advantage over sensing approaches involving the direct addition of C-dots to the water sample, as the target is concentrated and retained in the MIP film, while other chemicals can be removed by a simple cleaning step.

However, interference by other compounds in the sample could not be completely avoided. Figure 5.5 showed the $\mathrm{F}_{0} / \mathrm{F}$ ratios for both MIPs and NIPs, after placed in contact with a $10 \mathrm{ppm}$ DNT solution. The quenching effects increased slightly with $\mathrm{pH}$ for both MIPs and NIPs as $\mathrm{pH}$ turned more basic. Reasons for this trend may be the increased nonspecific adsorption at $\mathrm{pH}$ of 9 with higher swelling capacity, and acid or base residues after rinsing that may have been carried with the sensor. 


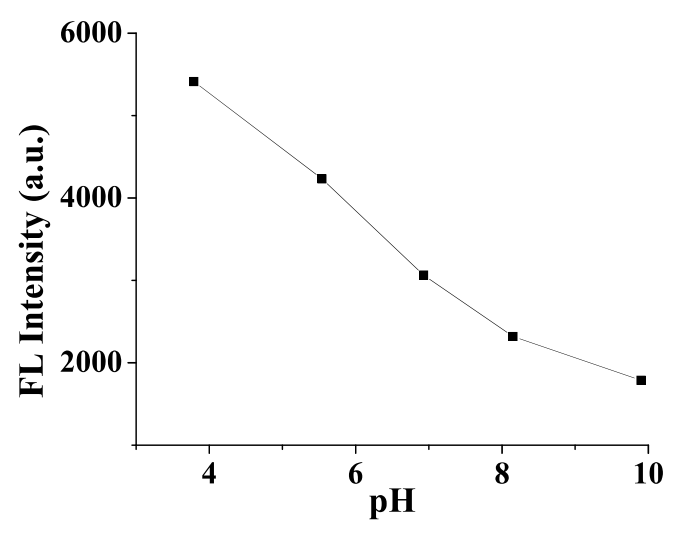

Figure 5.3 Fluorescence intensity of AC-Dots response to $\mathrm{pHs}$

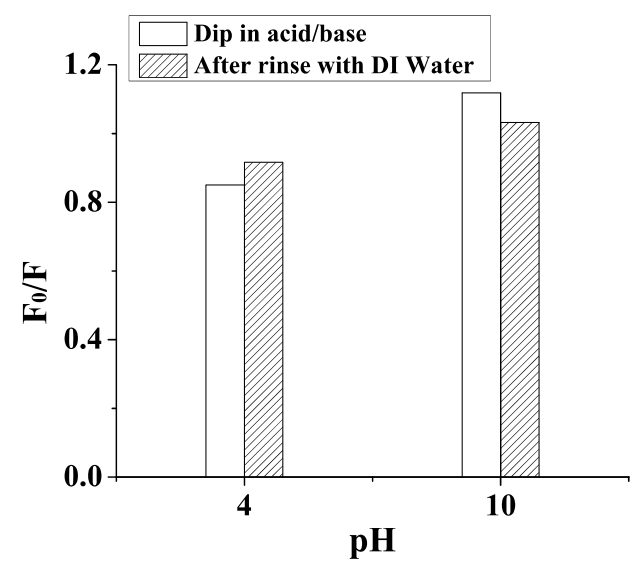

Figure 5.4 FL Intensity of MIP sensors measured in acidic $(\mathrm{pH}=4)$ and basic $(\mathrm{pH}=10)$ environments and measured after rinse in the absence of DNT.

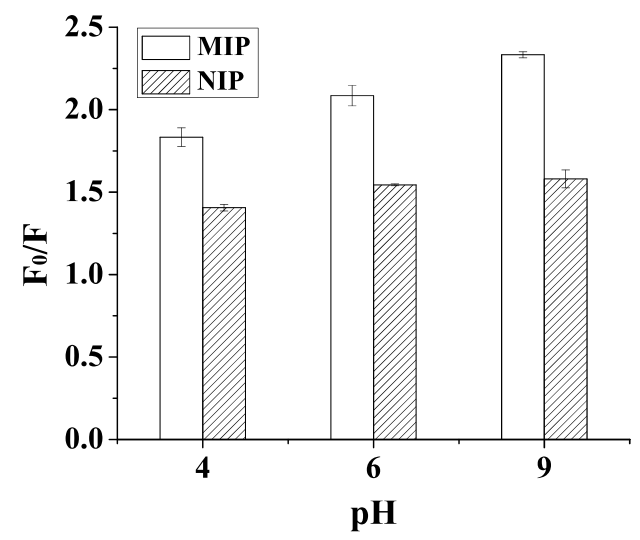

Figure 5.5 Quenching by DNT (10 ppm) at different pHs. 


\subsubsection{Effect of Ionic Strength and Different Salts Types}

The sensor was applied to the quantification of DNT solutions with variable dissolved salts concentration, resulting in a smaller FL intensity change for both MIP and NIP films (Figure 5.6). As expected, NIPs showed consistently less quenching amount by DNT than MIPs (Figure 5.6). With the increase in ionic strength from $1 \mathrm{mM}$ to $100 \mathrm{mM}$, the quenching amount of MIPs decreased $19 \%$ and $30 \%$ in the presence of $\mathrm{NaCl}$ and $\mathrm{CaCl}_{2}$, respectively. For NIPs, ionic strength from $1 \mathrm{mM}$ to $100 \mathrm{mM}$, the quenching amount decreased $25 \%$ and $38 \%$ with $\mathrm{NaCl}$ and $\mathrm{CaCl}_{2}$, respectively. Both cations decreased the binding of DNT; $\mathrm{Na}^{+}$is expected to have a more profound effect since cations were reported to have a significant influence on the binding capacity following the Hofmeister series (Kempe and Kempe 2010). However, we observed that a larger influence for $\mathrm{Ca}^{2+}$, which could be related to its chemical properties and its specific interactions with the AC-Dots surface (Bayati et al. 2017).

The diminishing gap between MIP and NIP quenching with increasing IS suggested that binding to the cavities may have also been affected. One possible explanation is that the specific adsorption based on the shape and size of cavities may decrease because of the conformational change of the hydrogels. It was reported that photonic polymers fabricated by colloidal crystal templating can swell or shrink in response to ionic strength variations (Huang et al. 2008). 


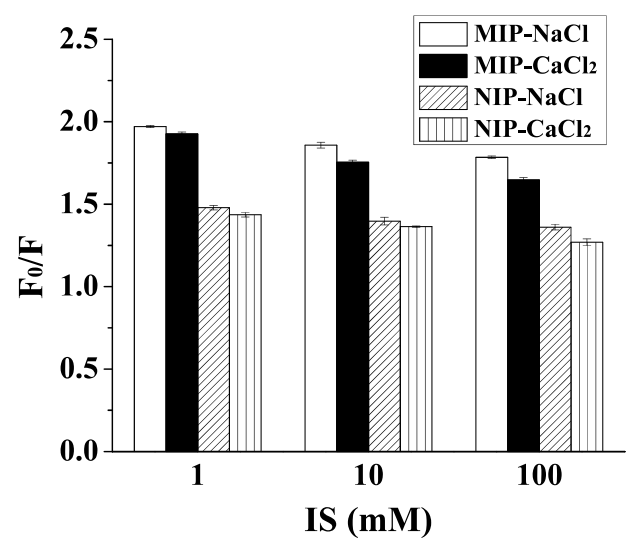

Figure 5.6 Quenching by DNT (10 ppm) with different salts, $\mathrm{pH}=6$

\subsubsection{Effect of NOM on Fluorescent Quenching}

Although their exact composition is variable, natural organic matter compounds are expected to be quenchers of AC-dots fluorescence and therefore may induce higher than real determinations by the sensor when used in natural waters (Lakowicz and Masters 2008). The quenching effect of SRNOM was first determined in the absence of DNT, in a range of concentrations plausible to be encountered in environmental samples. Figure 5.7 illustrated the decreased fluorescence intensity in the presence of SRNOM from a suspension of $0.2 \mathrm{mg} / \mathrm{L}$ AC-dots and from the MIP films. The fitting of the Stern-Volmer plot showed a linear relationship between $\mathrm{F}_{0} / \mathrm{F}$ and SRNOM concentrations for both ACDots and MIP systems (Figure 7). 


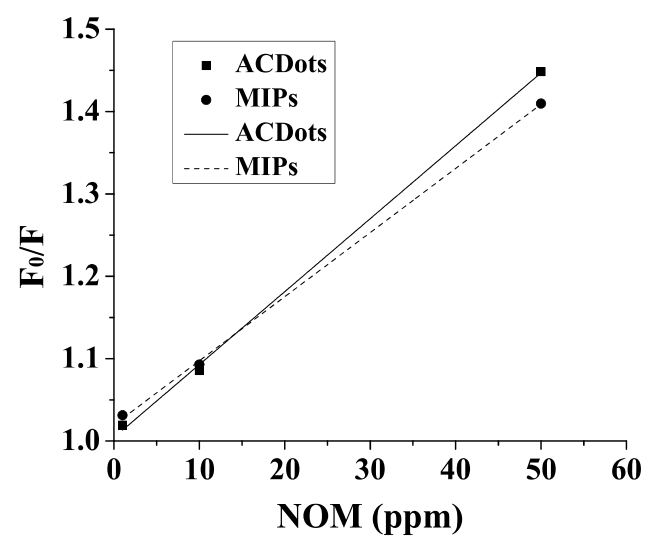

Figure 5.7 Quenching of AC-Dots and AC-Dots labeled MIPs by variable concentrations of SRNOM, in absence of DNT.

Consequently, the quenching amount increased when NOM existed in the DNT solutions (Figure 5.8) with respect to the pure water DNT solution. The slope of $\mathrm{F}_{0} / \mathrm{F}$ vs. DNT concentration curve for MIPs (0.0054) was very similar to that corresponding to the NIPs (0.0052), which implies that the increase in quenching was mainly related to nonspecific adsorption and not due to SRNOM occupying or blocking access to imprinted cavities. Since NOM did interfere with the specific capture of DNT molecules by the MIP, its concentration in a mixed solution with NOM could still be accurately obtained by subtracting the background quenching by NOM from the raw sensor reading. 


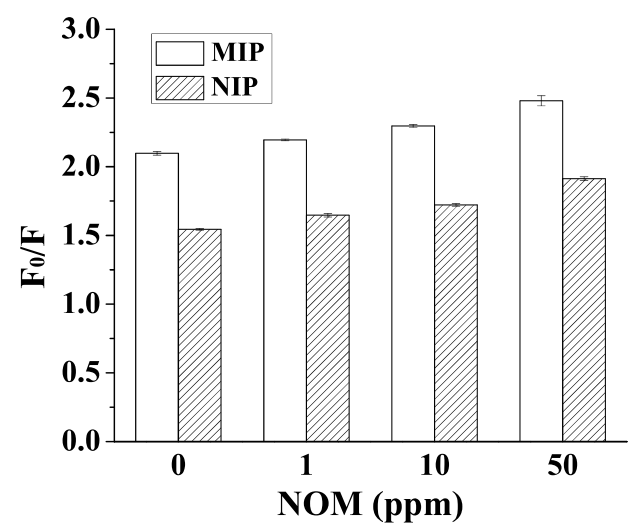

Figure 5.8 Quenching by DNT (10 ppm) with Suwannee River NOM

Equation 5.3 shows the response curve of the sensor in the absence of NOM (Dai et al. 2017):

$F_{o} / F=0.117 \times \mathrm{C}_{\mathrm{DNT}}+0.98551$

Equation 5.3

The NOM content of the sample can be estimated from the NIP reading (Figure 5.9) linear fit as shown by the following equation:

$F_{o} / F=0.0054 \times \mathrm{C}_{\mathrm{NOM}}+1$

Equation 5.4

The sensor calibration curve in the presence of NOM can be obtained combing Equations 5.3 and 5.4, resulting in: 


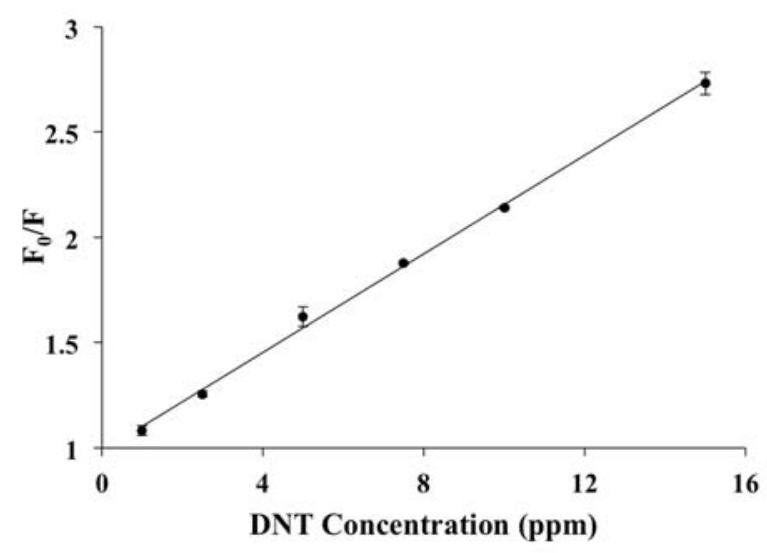

Figure 5.9 Stern-Volmer plot of AC-MIPs with an increasing concentration of DNT

$F_{o} / F=0.117 \times \mathrm{C}_{\mathrm{DNT}}+0.0054 \times \mathrm{C}_{\mathrm{NOM}}+1$

\section{Equation 5.5}

Applying the modified equation to the $10 \mathrm{ppm}$ DNT test solutions in the presence of NOM, values of 10.2, 10.6 and $10.3 \mathrm{ppm}$, were obtained by the sensors at SRNOM concentrations $1,10,50 \mathrm{ppm}$ respectively, proving the feasibility of ruling out the factor of NOM or other nonspecific quenchers based on the methods.

\subsubsection{Analytical applications in natural water samples}

Real natural water samples are complex solutions, where $\mathrm{pH}$, dissolved solids and natural organic compounds are probably the most frequent but not necessarily the only possible challenging components.

To further test the applicability of the sensor, the AC-MIP films were used for the determination of DNT concentrations in two real waters: Stephens Lake water and tap water from the laboratory at the University of Missouri. The collected samples were spiked with DNT and its concentration measured by the newly fabricated sensor and compared to HPLC analysis. 
The experiments were conducted for different DNT concentrations, and the corresponding analysis results are listed in Table 1. DNT concentrations were calculated using equation $5.5 . F_{o} / F=0.117 \times \mathrm{C}_{\mathrm{DNT}}+0.0054 \times \mathrm{C}_{\mathrm{NOM}}+1$

Lake water was slightly basic while tap water had a neutral $\mathrm{pH}$. The IS of both lake and tap water was close to $10 \mathrm{ppm}$, which was expected to have minor effects on the sensor performance. For the lake, the basic $\mathrm{pH}$ may result in underestimation of DNT concentrations, while medium IS could do the opposite. The TOC level of the lake water was higher than tap water due to the existence of dissolved NOM. Taking NOM in account and assuming $\mathrm{NOM}=\mathrm{TOC}-\mathrm{TOC}$ dnt, adjusted readings were obtained. The readings by sensors in tap water were closer to the HPLC analysis than in lake water. The sensor consistently underestimated the DNT concentration in the lake sample; in addition to the measured higher TOC content, lake water may have contributions of other unknown pollutants and particulate matter that can further interfere with the sensor detection. The matrix complexity results in a higher chance to block the cavities or the surfaces of sensor films. Still, the values in both tap and lakes were reasonably close to the HPLC measured DNT concentrations. The results are promissory, showing good recognition ability in challenging, unknown natural water samples.

Table 5.1 Detection of DNT in real water samples

\begin{tabular}{|c|c|c|c|c|c|c|c|}
\hline Sample & $\begin{array}{lr}\text { Sensor } & \text { measured } \\
\text { DNT } & \text { concentration } \\
(\mathrm{ppm}) & \end{array}$ & Stdev & Error (\%) & $\begin{array}{l}\text { HPLC measured DNT } \\
\text { concentration }(\mathrm{ppm})\end{array}$ & $\mathrm{pH}$ & $\begin{array}{l}\text { IS } \\
(\mathrm{mM})\end{array}$ & TOC (ppm) \\
\hline \multirow{3}{*}{ Lake } & 5.53 & 0.54 & 12.0 & 6.28 & \multirow{3}{*}{8.5} & \multirow{3}{*}{7.5} & \multirow{3}{*}{4.9} \\
\hline & 5.77 & 0.46 & 9.7 & 7.99 & & & \\
\hline & 12.32 & 1.86 & 18.4 & 14.67 & & & \\
\hline \multirow{3}{*}{ Tap } & 4.02 & 0.77 & 20.1 & 3.83 & \multirow{3}{*}{7.2} & \multirow{3}{*}{13.8} & \multirow{3}{*}{ Nondetectable } \\
\hline & 6.65 & 0.65 & 9.6 & 6.81 & & & \\
\hline & 10.66 & 0.69 & 6.0 & 11.49 & & & \\
\hline
\end{tabular}




\subsection{Conclusion and Future Study}

The effect of sample $\mathrm{pH}$, ionic strength and NOM content on the sensor performance was investigated. The influence on rebinding and fluorescence quenching process are concerns regarding the fabricated MIPs sensing ability. Surface chemistry of carbon dots can be affected by $\mathrm{pH}$, resulting in fluorescence emission change; nonspecific adsorption may be slightly higher at high $\mathrm{pH}$ with higher swelling capacity of the polymer. These effects result in higher quenching at basic environments, which means slightly higher readings than real DNT concentrations. At higher ionic strengths, the readings by sensors are relatively lower than real DNT concentrations. The decrease quenching effects with salts may be attributed to lower nonspecific adsorption, weaker specific binding, and less availability of imprinted cavities due to conformational change. In the presence of NOM, the readings observed were higher than expected based on actual DNT concentrations because NOM acted as a nonspecific quencher contributing to the quenching by DNT molecules. Since NOM quenching also obeys the Stern-Volmer equation, a modified expression including NOM concentration as a parameter was developed. For real water samples, the sensor was able to provide a rather accurate estimation of DNT concentration in tap and lake water, which was close to HPLC analysis. We believe that the AC-dots labeled DNT-MIP films fabricated in this work are promising materials for in situ detection of DNT contamination in the environment. 


\section{Chapter 6. Water Quality Monitoring at Hydraulic Fracturing Sites Using Molecularly Imprinted Porous Hydrogels}

The rapid rise in unconventional oil and natural gas (UOG) operations combined with directional drilling and hydraulic fracturing or fracking (HF) increases the opportunity for air and water pollution from these processes. UOG operations involve the injection of millions of gallons of water and thousands of gallons of more than 750 chemicals (Committee on Energy and Commerce 2011), which comes back to the surface as produced water with the addition of naturally occurring compounds such as radioactive materials, salts, arsenic, selenium, strontium, and barium which are embedded in the shale layer (Fontenot et al. 2013, Maule et al. 2013, Orem et al. 2014, The Shale Gas Subcommittee of the Secretary of Energy Advisory Board 2011, Warner et al. 2012). UOG operations release large amounts of reproductive, immunological and neurological toxicants, carcinogens, and endocrine disrupting chemicals (EDCs) into the environment (Colborn et al. 2010), and their health effects have been observed in intensively drilled areas in the United States (Bamberger and Oswald 2012).

Conventional methods to detect organic compounds involve not only expensive instrumentation, but also a large number of separating analytical procedures, resulting in a complex, time-consuming, and laborious screening procedure. Furthermore, detection in remote areas with difficult access adds complexity and inaccuracy due to sampling errors by non-trained personnel and sample conservation issues. Public health concerns stimulate the development of new analytical methods for the detection of contamination due to HF; in particular, approaches for easy and rapid detection of selected organic contaminants are highly desirable (Auriol et al. 2006, Matthiessen and Johnson 2006) . 
Molecular imprinting is a well-established technique used to synthesize polymers with specific molecular recognition nanocavities (Caykara et al. 2003). Owing to the complementarity in shape and binding sites, the created nanocavities exhibit high selectivity towards the imprinted molecules, including a large and diverse set of important organic compounds including hormones and metal ions (L. Chen et al. 2011, S. Wei et al. 2006, Zhu L et al. 2003).

Molecularly imprinted polymers (MIPs) were applied in the fabrication of sensors with capabilities to respond to a variety of physical or chemical stimulus; electrochemical, photoluminescent, and UV-vis spectroscopic sensors have been reported (Valero-Navarro et al. 2009, Yang et al. 2012a). Optical sensors based on the conformational change in presence of template are due to shrinking or swelling behaviors of MIPs. Inverse opal films of MIPs based on colloidal crystal template method have been reported (Griffete et al. 2012). These highly ordered structures made of hydrogels can swell or shrink in response to various stimuli including $\mathrm{pH}$, glucose, ionic strength, temperature and so on, leading to a change in reflection wavelength accompanied by a visually structure color change (Chen et al. 2016).

The objective of this work is to development of MIP based sensors for on-site sensing of UOG derived contamination. Sensors will be able to recognize the chemical fingerprint and would allow for rapid detection of impacts and quick adoption of mitigation strategies. This would limit the potential negative impact of UOG process, thus making it much more sustainable. 2BE has been identified in hydraulic fracking wastewater in high concentrations, up to grams per liter, and therefore can be considered an indicator of such contamination (Thacker et al. 2015). 
In this article, we report the development of a highly ordered porous photonic 2butoxyethanol-MIP film as UV-vis reflectance sensor. By combining MIP with inverse opals, the polymer is expected to selectively adsorb $2 \mathrm{BE}$ when present in the tested solutions, resulting in a change the conformational structure of the film and consequently a shift of the peak wavelength of the light reflected by the polymer (Wu et al. 2008b).

\subsection{Materials and methods}

\subsubsection{Materials}

Chemicals were purchased from Sigma-Aldrich and used without further purification; tetraethoxysilane (TEOS) (98\%), ammonia solution (25\% in water), ethanol (200 proof, ACS reagent, 99.5\%), acrylic acid (AA) (99\%), ethylene glycol dimethacrylate (EGDMA) (98\%), 2,2'-azobisisobutyronitrile (AIBN) (98\%), and 2-butoxyethanol (2BE).

\subsubsection{Molecularly Imprinted Porous Films Fabrication}

Monodisperse silica particles were synthesized following the Stöber method (Stöber et al. 1968). Briefly, $0.04 \mathrm{~mol}$ TEOS $(8.3 \mathrm{~mL})$ and ethanol $(200 \mathrm{~mL})$ were mixed in a round flask and stirred at $300 \mathrm{rpm}$. Then, ammonia $(18.3 \mathrm{~mL})$ was introduced and allowed to react for $12 \mathrm{~h}$. Silica particles obtained were centrifuged and redispersed twice in DI water and once in ethanol for purification. Colloidal crystals were fabricated by vertical self-assembly on a glass substrate as described elsewhere (Jiang et al. 1999).

Polymerization mixtures were prepared from $0.8 \mathrm{~mL}(11.6 \mathrm{mmol})$ acrylic acid (AA) as the functional monomer, $0.55 \mathrm{~mL}(2.9 \mathrm{mmol})$ ethylene glycol dimethacrylate (EGDMA) as the crosslinker agent, $6 \mathrm{mg}(0.04 \mathrm{mmol})$ azobisisobutyronitrile (AIBN) as the initiator, 
and $4 \mu \mathrm{l}(0.03 \mathrm{mmol}), 40 \mu \mathrm{l}(0.30 \mathrm{mmol}), 0.1 \mathrm{~mL}(0.76 \mathrm{mmol}) 2 \mathrm{BE}$ as the target with a molar ratio of target molecule: functional monomer: crosslinker of 1:387:97, 1:39:10, and 1:15:4, respectively. PMMA slides were placed on both sides of the glass substrate with the colloidal crystal and the assembly was held firmly together. One end of the slide assembly was put in contact with the polymerization mixture so that, as the liquid raised by capillary forces, it filled the void spaces within the colloidal crystal. The polymerization was performed under UV light at wavelength of $365 \mathrm{~nm}$ for $4 \mathrm{hrs}$ at room temperature. Silica particles were removed by submerging in 5\% hydrofluoric (HF) acid bath. Finally, to remove the target molecule, the resulting polymer film was dried in a vacuum oven (Accu Temp-09, Across International) at $40{ }^{\circ} \mathrm{C}$ and $30 \mathrm{mmHg}$ (30 Torr/4000Pa) for $10 \mathrm{hrs}$. The fabrication process is demonstrated in Figure 6.1. The fabrication of non-imprinted polymers (NIPs) followed a similar procedure but without the addition of $2 \mathrm{BE}$ to the polymerization solution. 

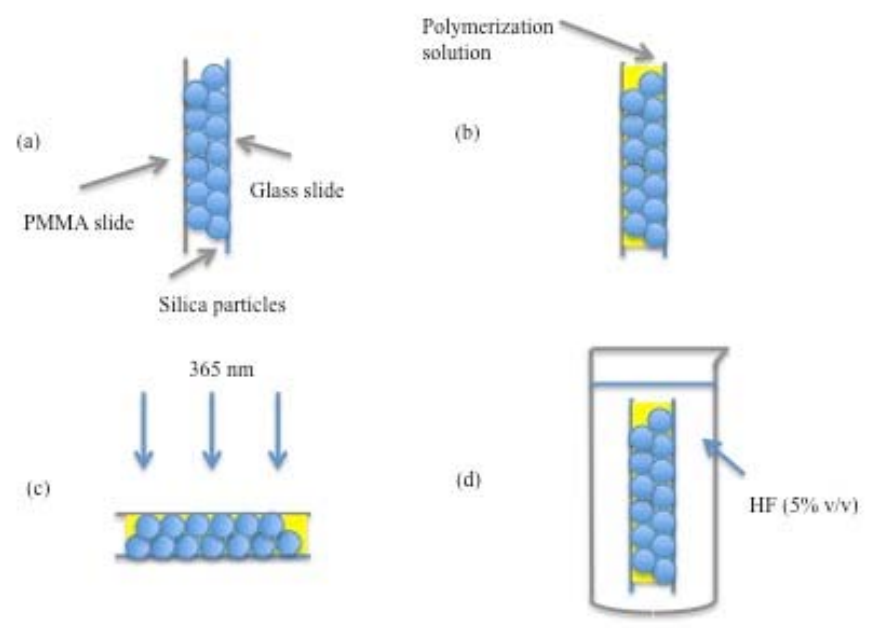

(e)
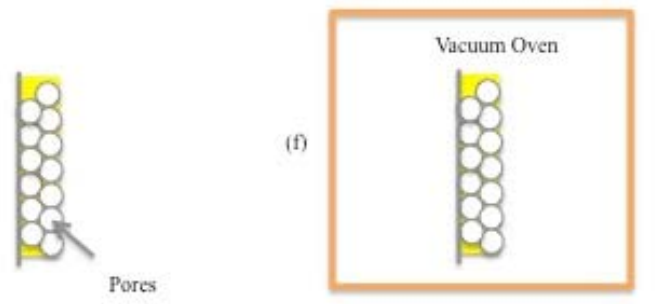

Figure 6.1 Schematic of the MIP fabrication process: (a) monomer infiltration of the colloidal silica deposit; (b) glass "sandwich" formation; (c) UV polymerization; (d) particle and glass removal by hydrofluoric acid; (e) porous film containing target molecule; (f) removal of $2 \mathrm{BE}$ molecules by thermal treatment in vacuum to obtain MIP.

Similarly, assemblies made with all three glass slides led to self-standing films after HF treatment. The self-standing films were prepared for characterization of the material and equilibrium rebinding experiments with the target molecule: functional monomer: crosslinker of 1: 387: 97. PMMA supported films were used in reflection experiments.

\subsubsection{MIPs Characterization}

The particle size distribution (PSD) of silica particles was analyzed using dynamic light scattering (DLS) on a Zetasizer Nano ZS instrument (Malvern Instruments). Additionally, silica particle deposits and porous films were imaged by a FEI Quanta 600 FEG 
Environmental Scanning Electron Microscopy (ESEM). Samples were attached to a metal mount using carbon tape and were coated with a thin layer of platinum to provide a conductive surface using a sputter coater (Emitech K575x). The SEM images were analyzed, using the software Image J (Schneider et al. 2012b): over 300 particles were measured from at least 5 different images.

Hydrogel film swelling experiments were performed in phosphate buffer at three different $\mathrm{pH}$ values $(4,7$ and 10$)$ at room temperature. The polymers were swollen in solutions for $48 \mathrm{~h}$ at ambient temperature and the degree of swelling was determined gravimetrically. The percentage swelling ratio $(\% \mathrm{SR})$ was calculated from the expression

$\% S R=\left(m_{s}-m_{d}\right) / m_{d}$

Equation 6.1

where $m_{s}$ is the mass of the swollen film at equilibrium and $m_{d}$ is the mass of the freeze dried films.

Thermal properties of the NIP-PAA films were determined by the differential scanning calorimetry (DSC) technique. The analysis was carried out on a TA Instruments Q100 Differential Scanning Calorimeter, at a heating rate of $5{ }^{\circ} \mathrm{C} / \mathrm{min}$ between $40{ }^{\circ} \mathrm{C}$ to $120^{\circ} \mathrm{C}$. The sample ( $\sim 2 \mathrm{mg}$ of a lyophilized hydrogel) was sealed into a DSC aluminum pan, and an empty aluminum pan was used as reference.

\subsubsection{Binding / Incubation tests}

Incubation tests were conducted by suspending approximately $6-10 \mathrm{mg}$ of dried (MIPPAA, NIP-PAA) unsupported films in a $20 \mathrm{~mL}$ 2BE solution for 2 days, after which the system was assumed to reach adsorption equilibrium. Then, the films were separated and solutions sampled for analysis. Tests were performed at 2BE initial concentrations of 10 , 
$20,35,45$, and $60 \mathrm{ppm}$.

The recognition capacity ( $\mathrm{RC}$ ) is defined as the adsorbate mass per unit of adsorbent mass and is calculated from the equation:

$R C=\left(C_{i}-C_{e}\right) V / m$

Equation 6.2

where $C_{i}$ and $C_{e}$ are the initial and the equilibrium concentrations of the DNT in solution (in $\mathrm{mg} \mathrm{mL}^{-1}$ ), respectively, $V$ is the volume of solution (in $\mathrm{mL}$ ) and $m$ is the mass of the films. Then, the imprinting efficiency (IE) is defined by

$I E=R C_{M I P} / R C_{N I P}$

Equation 6.3

where $\mathrm{RC}_{\mathrm{MIP}}$ and $\mathrm{RC}_{\mathrm{NIP}}$ are the recognition capacities of MIP and NIP-PAA, respectively. GC-MS Analysis

Recognition capacity (RC) and imprinting efficiency (IE) were calculated based on the concentration measured by liquid-liquid extraction followed by GC-MS. In brief, $2 \mathrm{BE}$ in aqueous solution was extracted using chloroform in the ratio of 1:1 (v/v). The mixture was vortexed for 1 minute, and centrifuged at 4000 RPM for 5 minutes. The lower layer was separated, and filtered through a $0.2 \mu \mathrm{m}$ membrane filter (Whatman Anotop 10) prior to injection into Varian 3400CX GC with a Hewlett Packard cross-linked methylsiloxane DB-5 capillary column (30 m x 0.25 mm I.D.) coupled with a Varian Saturn 2000 iontrap mass selective detector (Varian Inc., Walnut Creek, CA). The GC temperature program was initially set at $35^{\circ} \mathrm{C}$ for 5 minutes, then increased to $250^{\circ} \mathrm{C}$ at $10^{\circ} \mathrm{C} / \mathrm{min}$, and was held for 10 minutes, using split injection mode with split ratio of 1:100, and constant carrier gas flow $(\mathrm{He}, 1.0 \mathrm{~mL} / \mathrm{min})$. Injector temperature was held at $275^{\circ} \mathrm{C}$. Transfer line between the GC and mass spectrometer was held at $280^{\circ} \mathrm{C}$, and the ion trap manifold was set to $250^{\circ} \mathrm{C}$. The quantitative ions were selected by injecting a standard solution of $2 \mathrm{BE}$, 
and selection of diagnostic and quantitative ions was optimized by a procedure previously described (Lin et al. 2007). Calibration curves were constructed using seven concentration levels of $2 \mathrm{BE}$ standards to represent relationship between known concentrations and ion intensities.

\subsubsection{Optical sensing tests}

Due to the uniformly ordered porous structure, the reflection peaks recorded by the spectrophotometer could be interpreted as interferences between light reflection on dense (111) plane (Griffete et al. 2012). The peak, $\lambda_{\max }$, for the porous hydrogel is related to the structure of the porous film by the Bragg equation:

$\lambda_{\max }=1.633(\mathrm{~d} / \mathrm{m})\left(\mathrm{D} / \mathrm{D}_{0}\right)\left(\mathrm{n}_{\mathrm{a}}^{2}-\sin \theta^{2}\right)^{0.5}$

Equation 6.4

where $d$ is the sphere diameter of the silica colloidal particles used to fabricate the porous film, $m$ is the order of Bragg diffraction, $\left(D / D_{0}\right)$ is the degree of swelling of the gel $(D$ and $D_{0}$ denote the diameters of the gel in the equilibrium state at a certain condition and in the reference state, respectively), $n_{a}$ is the average refractive index of the porous gel at a certain condition, and $\theta$ is the angle of incidence.

Optical sensing tests were conducted for MIPs (with three molar ratios of target molecule: functional monomer of 1: 387, 1:39, and 1: 15) and NIP films. The kinetic experiments were conducted with an initial concentration of $10 \mathrm{ppm}$. In the sensor performance tests, supported films were exposed to $20 \mathrm{ml} 2 \mathrm{BE}$ solutions at concentrations of $0,1,10,100$ ppb and 1, 10, 100 ppm for 30 mins, which is sufficient for shifts based on the kinetic tests, and excess solutions remained on the films were wiped before reading the reflectance. 
Reflectance of the photonic hydrogel films was measured over a wavelength range of 200-800 nm, using a double-beam UV-Vis-NIR spectrophotometer (Cary 60, Varian) with a Harrick Scientific's Specular Reflection Accessory (ERA-30G) for measurement reflectance at a fix angle of 30 degrees.

\subsection{Results and Discussion}

\subsubsection{Characterization of MIPs}

The digital SEM images (Figure 6.2 a) of silica particle deposits were analyzed, and the average particle size obtained from microscopy images was $213 \pm 9 \mathrm{~nm}$, while DLS determinations yielded an average size of $231 \pm 1 \mathrm{~nm}$. The two techniques showed excellent agreement, taking in consideration that DLS measures hydrodynamic radius of the particle, and it usually overestimates the actual size.

The colloidal crystals acted as a template for the porous structure and therefore a highly organized, open structure was obtained (Figure 6.2 b and c). This porous macrostructure can provide a high surface area for easy access to abundant binding sites. 


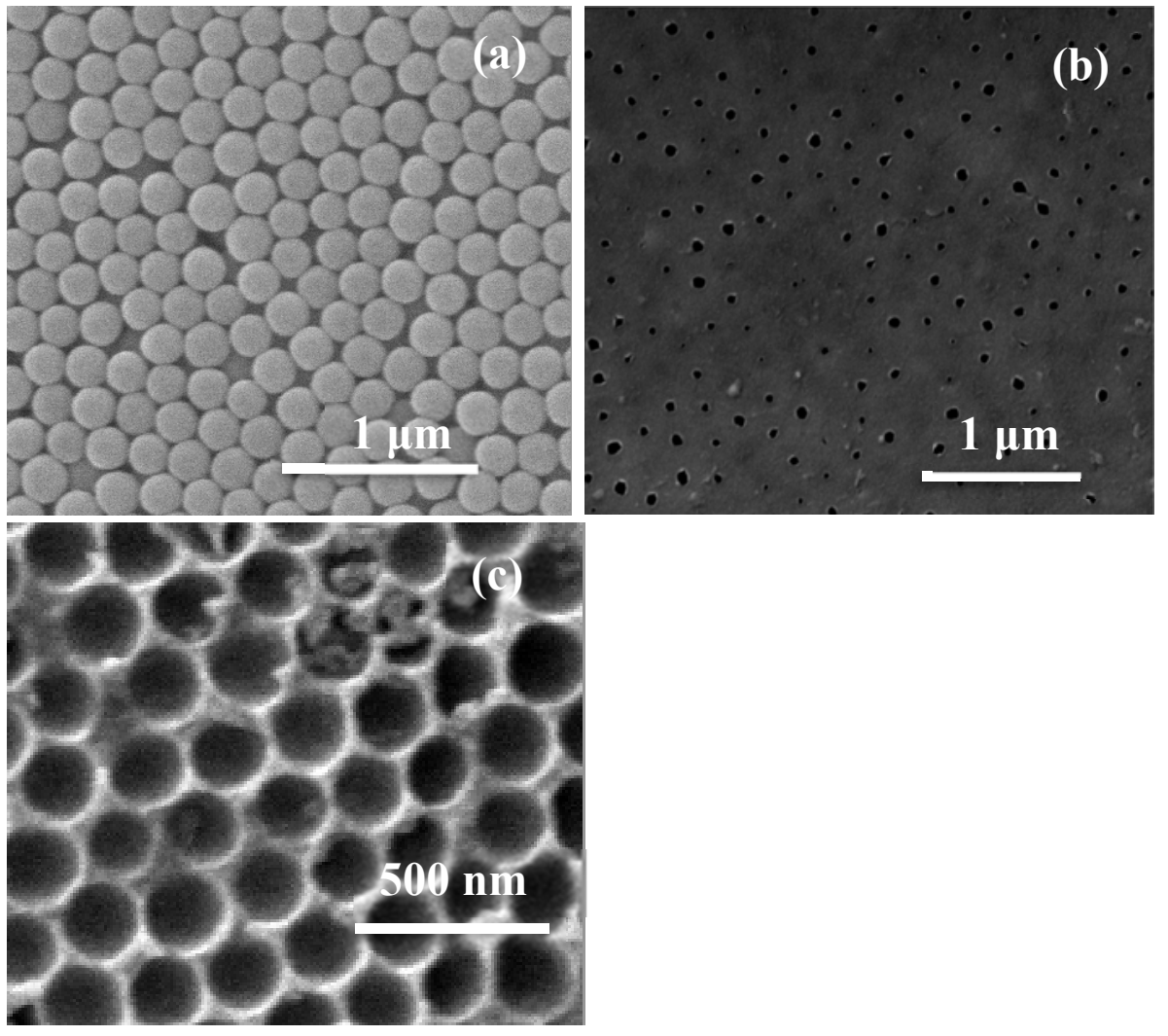

Figure 6.2 SEM images of: silica particles (a); porous film surface (b); inner structure (c).

The DSC scan showed that the films went through a glass transition process at a temperature (Tg) of $50.3{ }^{\circ} \mathrm{C}$ (Figure 6.3). Therefore, the polymer films are stable and safe to use and recycle under $50.3{ }^{\circ} \mathrm{C}$, which also determines that the template removal heating temperature needs to be below the glass transition temperature to maintain the integrity and property of polymer films. 


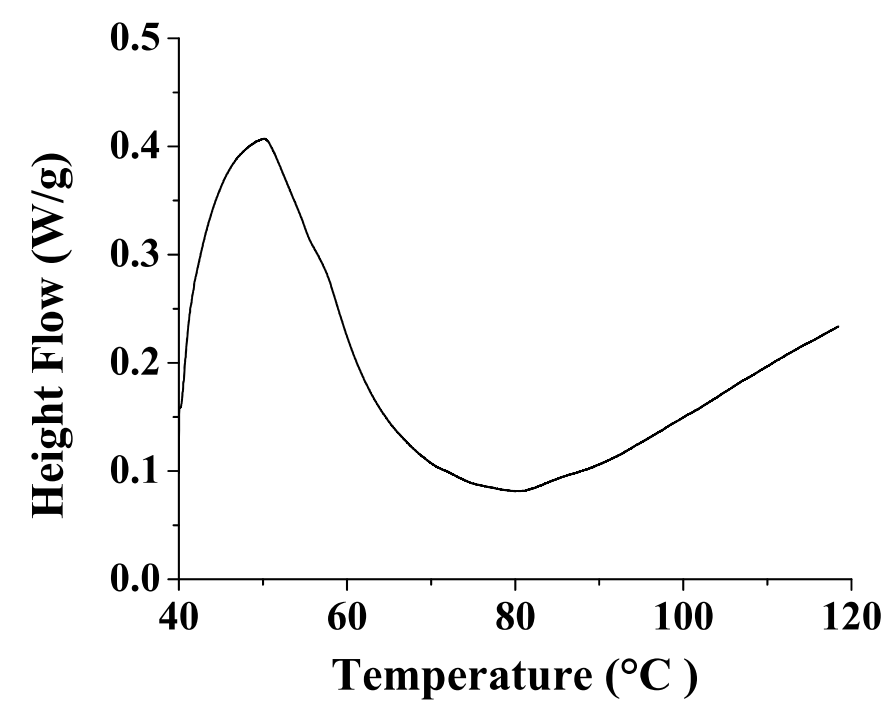

Figure 6.3 DSC thermograms of NIP hydrogel films.

Figure 6.4 shows the \% SR as a function of $\mathrm{pH}$ for the unsupported films. At low $\mathrm{pH}$ values, carboxyl groups present in the polymer reduced the repulsive interactions between the polymer chains, resulting in less swelling. In contrast, at neutral and alkaline $\mathrm{pH}$ values when carboxyl groups become deprotonated, electrostatic repulsion together with the breaking of hydrogen bonds generated an increase of the material swelling. Therefore, $\mathrm{pH}$ must be kept constant for sensor reading to be comparable, since the reflectance may change with $\mathrm{pH}$. 


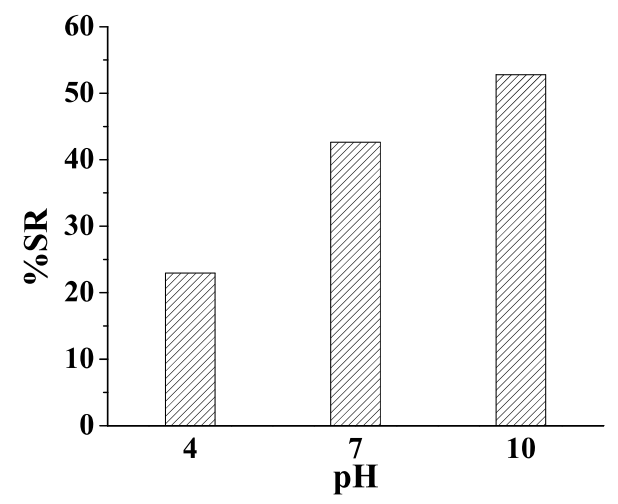

Figure 6.4 Swelling ratio (\%SR) variations of PAA films with $\mathrm{pH}$.

\subsubsection{Recognition Capacities of MIPs and NIPs}

The recognition capacities of the 2BE-MIPs and NIPs were investigated through affinity adsorption experiment. Figure 6.5 shows the binding performance of the 2BE-MIPs and NIPs at different concentrations of $2 \mathrm{BE}$ at neutral $\mathrm{pH}$. In all of the concentrations studied, the MIP exhibited higher adsorption capacity of 2BE than the NIPs and also binding increased at the higher rate for MIPs than NIPs, with imprinting efficiency (IE) of approximately 2; from 10 to $35 \mathrm{ppm}$, the IE increased from 2.0 to 2.2 , and from $35 \mathrm{ppm}$ to $60 \mathrm{ppm}$, the IE decreased from 2.2 to 1.9 . After a certain concentration, the specific binding capacity of the film can be saturated, and then IE is expected to decrease. The higher adsorption of MIP is the result of a combination of specific adsorption of $2 \mathrm{BE}$ due to the cavities provided by the molecularly imprinting and nonspecific absorbed molecules loosely bound on the surface of polymer films, not captured by the imprinted cavities. In contrast, only nonspecific adsorption may occur in the NIP films. In the range of concentrations investigated, NIPs exhibited relatively linear increase of recognition capacity with concentration while MIPs seemed saturated after $35 \mathrm{ppm}$ due to the limited amounts of specific sites available. Moreover, RC of MIPs changed at a faster rate than 
NIPs at lower concentrations, because of a higher affinity of 2BE towards the MIPs.

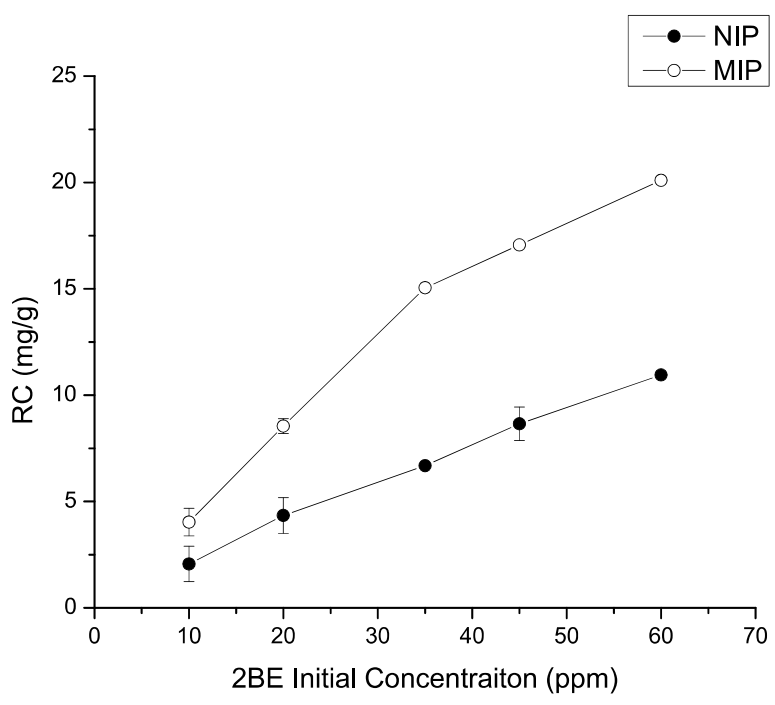

Figure 6.5 Recognition capacities of NIP and MIP at neutral pH.

\subsubsection{Sensing properties of MIPs}

According to the Bragg equation, if the molecular recognition process could cause any change in the pore structure of the prepared hydrogel, a readable optical signal may be detectable. Due to the 3D ordered porous structure, the signal can be generated by the molecularly imprinted hydrogel itself through Bragg diffraction, and the molecular recognition process can be directly transferred into readable optical signals.

The hydrogels showed well defined Bragg's peaks. It was found that the optical diffraction of MIPs was very sensitive to the rebinding of butoxylethanol (2BE) molecules. Upon exposure to $2 \mathrm{BE}$, the diffraction peak wavelength changed; with the increase of the concentration of $2 \mathrm{BE}$ in the test solution, the peak shifted gradually to longer wavelengths (Figure 6.6). To elucidate the molecular recognition properties of the 
imprinted materials, a non-imprinted 3D ordered macroporous hydrogel (NIP) as a control sample was also synthesized under the same chemical conditions as the imprinted hydrogel, but in the absence of $2 \mathrm{BE}$ template. Figure $6 \mathrm{~d}$ displays the evolution of the diffraction peak of the NIP hydrogel film upon exposure to $2 \mathrm{BE}$ solutions with different concentrations. Only slight fluctuations of the Bragg peak were observed in the case of the non-imprinted hydrogel film, probably due to some nonspecific adsorption, but the response was remarkable different from that exhibited by MIP film.

For the same $2 \mathrm{BE}$ concentration, smaller wavelength shifts were seen at low template/monomer ratios (1:387); wavelength shifts increased with higher template/monomer ratios (1: 39 and 1:15). However, when the monomer/template was high enough (Figure 6.7) the shifts responding to the same concentrations were similar (at template/monomer ratios of 1:39 and 1:15). This result indicates that for the 1:39 ratio, the MIP films gained enough cavities that availability of specific sites was not a limiting factor in the binding, and the film was able to efficiently transfer the swelling performance to reflectance signal changes. For these three MIPs, the shifts seemed to vary linearly with logs of concentration. Among them, MIP2 displayed the best fit, presented in Equation 5, with an $\mathrm{R}^{2}$ of 0.989 .

$y=8.90 \times \log x+5.32$

Equation 6.5

The shift observed for the NIPs, which can be associated with nonspecific adsorption, was used for the detection limit calculation. Therefore, the detection of limit of MIP2 is $3.4 \mathrm{ppm}$. 
Overall, the results demonstrated that the wavelength shift remains fairly unaffected by nonspecific interactions, although the binding experiments indicated they occurred. The existence of nonspecific adsorption is one of the main challenges of MIPs. The developed sensor presented the noteworthy advantage of minimizing its effect by a combination a superior transduction mechanism, mainly unaffected by non-specific binding, and the ability of the sensor slide to be rinsed before reflectance measurement so to get rid of some loosely bound material, neutralizing other potentially interfering effects, as for example $\mathrm{pH}$ variation or complex water matrixes.
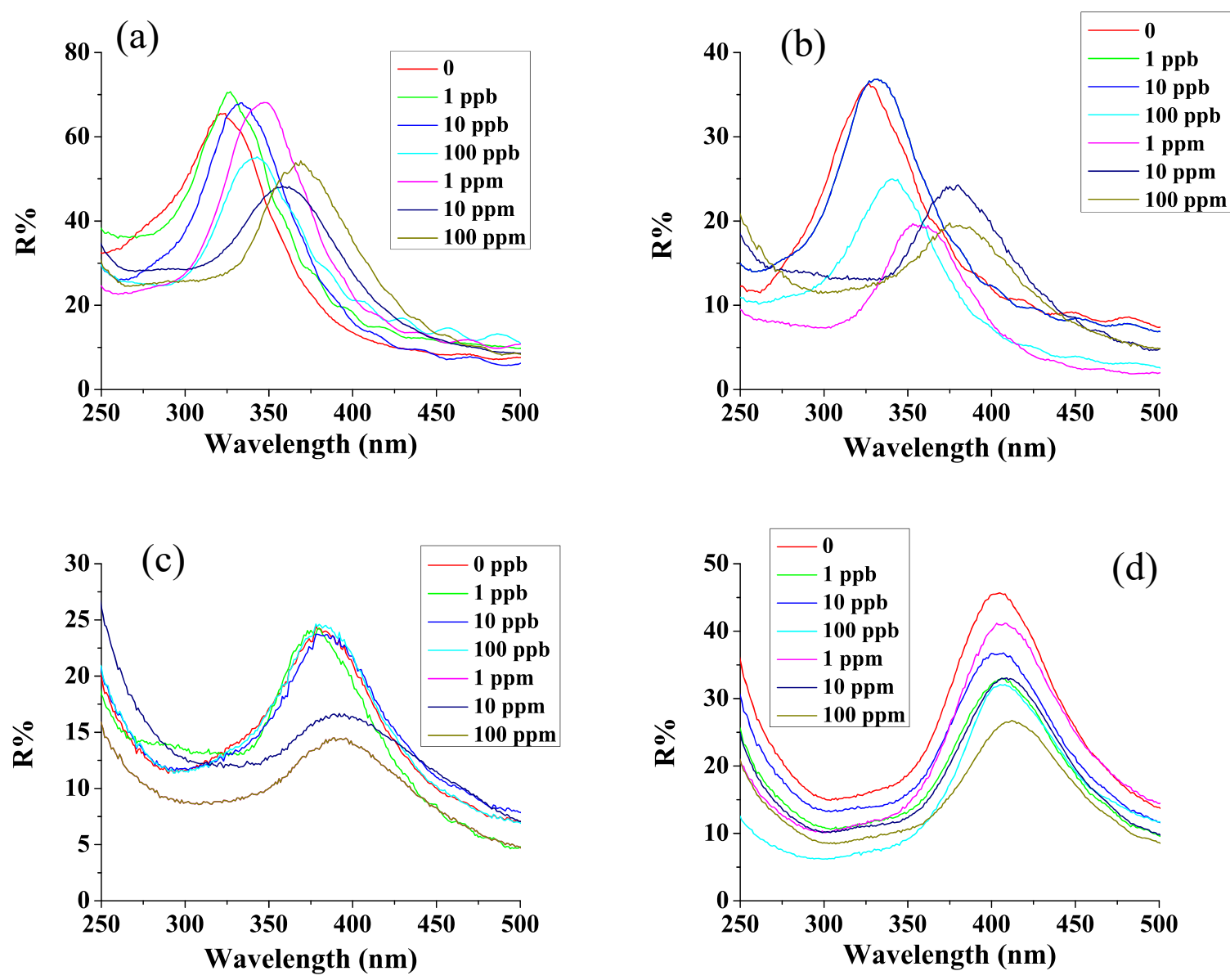
Figure 6.6 Reflectance spectra at $\theta=30^{\circ}$ of a: MIP1 (molar ratio of template/monomer $=1: 15$ ), b: MIP2 $($ molar ratio of template/monomer $=1: 39), \mathrm{c}:$ MIP3 $($ molar ratio of template/monomer $=1: 387)$, and d: NIP porous film.

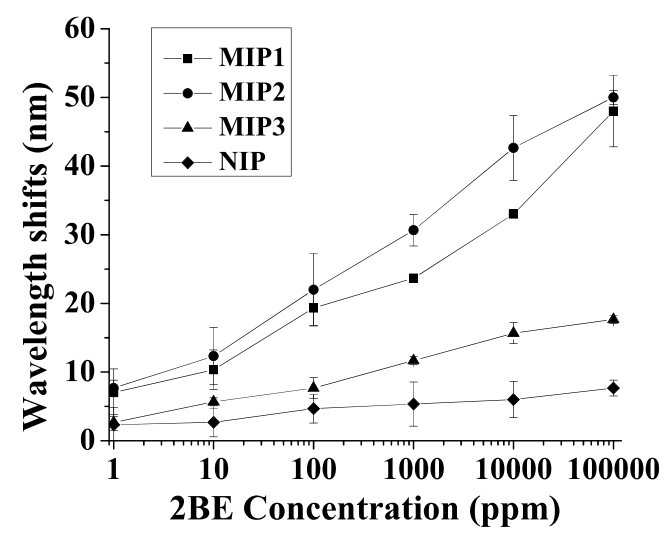

Figure 6.7 Wavelength shifts of MIP1, MIP2, MIP3 and NIP.

The sensor had a fast response to the presence of 2BE. Figure 6.8 shows the reflectance shifts in the first 25 minutes, and remained the same after 30 minutes. Therefore, we chose 30 minutes for all the tests.

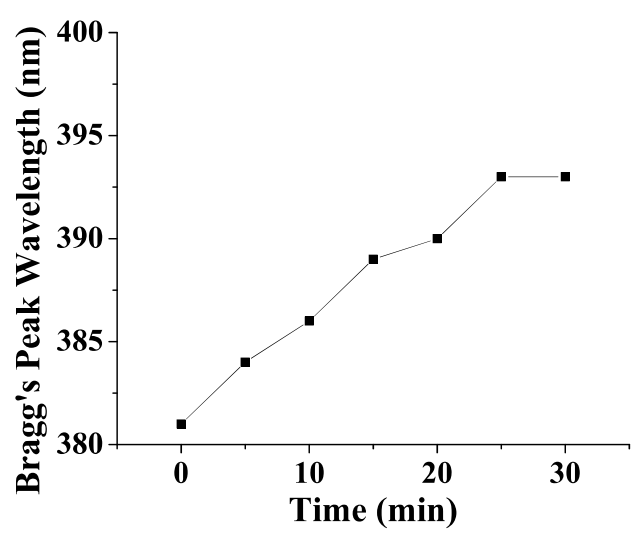

Figure 6.8 Bragg's peak wavelength response time of MIP sensors for 2BE with initial concentration of 10 ppm. 
The economic feasibility of the sensor as well as its practicality is strongly related to the ability to regenerate and reuse the films. Theoretically, the same approach employed to remove the target molecule after fabrication would result in successful regeneration. However, besides the well-known template and monomer leaching, polymer degradation products can also result in contamination of samples during the application of the MIPs. We investigated the reusability of the sensor conducting 5 cycles of 2BE removal (by vacuum oven at $40{ }^{\circ} \mathrm{C}$ and $30 \mathrm{mmHg}$ for $10 \mathrm{hrs}$ ) and incubation for 30 minutes in $100 \mathrm{ppb}$ 2BE solutions. The results revealed the sensor had good reusability, with a slightly increase of $5 \mathrm{~nm}$ for initial Bragg's peak wavelength (Figure 6.9).

The MIPs sensor has a shelf life of at least 3 months, during which period it exhibited a constant and well defined Bragg's peak wavelength (327 nm for MIP1); the materials showed to be stable, and can be stored indefinitely in dry form at normal ambient conditions.

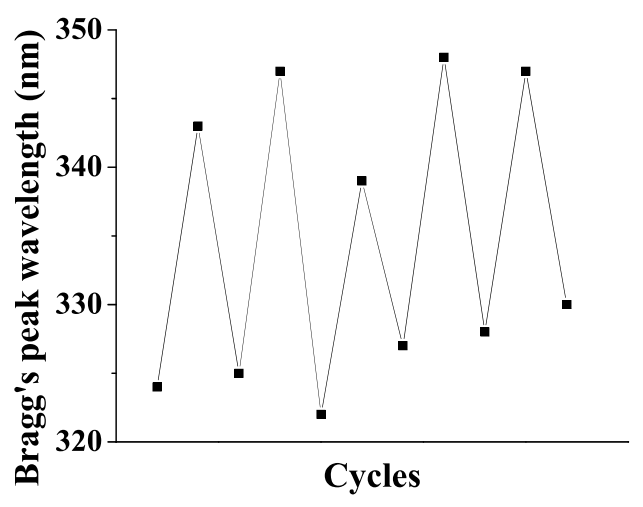

Figure 6.9 Reusability test of MIP sensor (2BE concentration $=100 \mathrm{ppb})$.

\subsection{Conclusion}

The sensor porous morphologies were successfully obtained by bulk polymerization 
inside a colloidal crystal void space. Molecularly imprinting with $2 \mathrm{BE}$ did not alter the structure of the film, as evidenced by the results of the characterization work on imprinted and non-imprinted films. The MIPs exhibited higher adsorption capacity towards 2BE than the NIPs, with IEs around 2. The higher adsorption of MIP is the result of a combination of specific adsorption of $2 \mathrm{BE}$ due to the cavities provided by molecularly imprinting and nonspecific adsorption. A readable optical signal of the reflectance Bragg's peak position shifts of sensor is related to the $2 \mathrm{BE}$ concentrations from $1 \mathrm{ppb}$ to $100 \mathrm{ppm}$. From the shift number, we can estimate the $2 \mathrm{BE}$ concentration from unknown samples, and potentially evaluate the $2 \mathrm{BE}$ levels in surface water or groundwater.

The sensor can be used for the concentration range from 10 to $10000 \mathrm{ppm}$ for quantitative analysis, with a LOD of $3.4 \mathrm{ppm}$. The non-specific adsorption did not show a significant interference effect. The sensor exhibited a good reusability for 5 cycles of binding and removing of the target and had a shelf life of at least 3 months. The results indicated that it is stable over time and multiple uses, making it a sustainable and cost effective approach for $2 \mathrm{BE}$ analysis in water.

The availability of early detection of hydraulic fracking contamination is the main benefit to people and the planet from a human health perspective; secondly, and as a direct consequence of the former, the sustainable exploitation of a natural resource in the US and around the world has important implications for society development, economic growth and international relationships. 


\section{Chapter 7. Conclusions and Future Study}

In this study, we have explored carbon dots with different surface functionality. Their surface charge, given by the zeta potential, and their hydrodynamic diameter in suspension were investigated under a variety of environmentally relevant conditions. The results showed that water chemistry altered the surface charge of the nanomaterials, but their hydrodynamic size could not be correlated to those changes. The aminofunctionalized carbon dots (AC-dots) were applied to fluorescently label a molecularly imprinted polymer (MIP) for 2,4-dinitrotoluene (DNT) as a template. MIPs exhibit higher adsorption than NIPs, with imprinting efficiencies ranging from 2 to 2.5. DNT is specifically captured by the cavities in the MIP and interact with AC-dots on the surface, resulting in quenching of the fluorescence of the AC-dots. Response to DNT reaches equilibrium within $\sim 30 \mathrm{~min}$. The method has a dynamic range that extends from 1 to 15

ppm, and allows for quantitation of DNT in aqueous solutions, with a detection limit of $0.28 \mathrm{ppm}$. Selectivity tests conducted in presence of DNT analogs demonstrated the specific recognition of DNT. We investigated the sensor performance in various aqueous matrixes to understand the effect of water chemistry on C-MIPs fluorescence quenching and affinity. The results suggest that with the increase of ionic strength from $1 \mathrm{mM}$ to $100 \mathrm{mM}$, the quenching amount of MIPs decreased about $19 \%$ and $30 \%$ with $\mathrm{NaCl}$ and $\mathrm{CaCl}_{2}$ respectively. In the range of $\mathrm{pH}$ from 4 to 9 , quenching effect is slightly higher at basic environment for both MIPs and NIPs resulting from swelling properties of the films. In both lake water and tap water, DNT concentrations read by the sensors were very close to the HPLC measured DNT concentrations with the range from $76 \%$ to $104.9 \%$. Molecularly imprinted polymers (MIPs) sensors for detection of 2-butoxyethanol (2BE), 
which presence in water is associated with hydraulic fracturing contamination, were developed based on the combination of a colloidal crystal templating method and a molecular imprinting technique. MIPs exhibited higher adsorption than NIPs due to the specific adsorption provided by molecular imprinting with imprinting efficiencies around 2. The reflection spectra of the sensors showed Bragg's peaks, which responded to the presence of 2BE. The reflectance changes showed increasing red shifts up to $50 \mathrm{~nm}$ with $2 \mathrm{BE}$ concentrations in the range of $1 \mathrm{ppb}$ to $100 \mathrm{ppm}$, which allows quantitative estimates of present $2 \mathrm{BE}$ concentration in aqueous solutions.

In summary, MIP based sensors were applied to detect DNT/2BE based on fluorescence/reflectance properties. They are able to quantitatively analyze the concentration of target contaminants and have the potential to use in real water and early detection to certain process by fingerprinting.

We will investigate the effect of analogues and potential interference from natural organic matter or dissolved substances in natural waters for photonic MIP sensors. Selectivity studies of MIPs will be carried out in a similar way that the recognition studies for each target molecule, but in this essay MIPs will be incubated in the presence of the target and an appropriate analogue, i.e. a molecule structurally similar to the target that may be present in the water matrix, in equal molar concentration. Furthermore, interference studies will be conducted in similar fashion, by incubation of the MIPs with the target in a modified solvent that would mimic natural water conditions. Suwannee River humic acid (SRHA) will be used as a model for dissolved organic matter; $\mathrm{NaCl}$ and $\mathrm{CaCl}_{2}$ will be added to provide a background ionic strength, representing waters with a range of total dissolved solids and hardness levels. All selectivity studies will be carried 
out in duplicate. Two water chemistries will be considered: groundwater and surface water. The former is characterized by higher dissolved solids concentration and above neutral $\mathrm{pH}$, while the latter is generally associated with presence of dissolve organic matter and lower $\mathrm{pH}$ values.

The long-term goal is to develop a sensing device composed of an array of MIPs individually designed to capture the group of contaminants identified in hydraulic fracking process, that could be deployed at potentially impacted or at risk sites to monitor surface and /or groundwater contamination by hydraulic fracturing activity. Each film within the sensor should produce a measurable detection signal proportional to the concentration of the pollutant in the water and should store or transmit the information to a centralized location for effectively water quality monitoring of over large areas. Optical methods, including fluorescence by addition of photoluminescent nanoparticles to the porous films, are preferred, but other approaches like piezometric (Dickert et al. 2004) or photonic sensors are possible (Griffete et al. 2011b). 


\section{References}

(2014) Technical Fact Sheet - Dinitrotoluene (DNT), United States Environmental Protection Agency

Alizadeh, T., Zare, M., Ganjali, M.R., Norouzi, P. and Tavana, B. (2010) A new molecularly imprinted polymer (MIP)-based electrochemical sensor for monitoring 2,4,6-trinitrotoluene (TNT) in natural waters and soil samples. Biosensors and Bioelectronics 25(5), 1166-1172.

Attarchi, M.S., Ashouri, M., Labbafinejad, Y. and Mohammadi, S. (2012) Assessment of time to pregnancy and spontaneous abortion status following occupational exposure to organic solvents mixture. Int Arch Occup Environ Health 85(3), 295303.

Auriol, M., Filali-Meknassib, Y., Tyagia, R., Adamsc, C. and Surampallib, R.Y. (2006) Endocrine disrupting compounds removal from wastewater, a new challenge. Process Biochemistry 41(3), 525-539.

Baker, S.N. and Baker, G.A. (2010a) Luminescent Carbon Nanodots: Emergent Nanolights. Angew Chem Int Edit 49(38), 6726-6744.

Baker, S.N. and Baker, G.A. (2010b) Luminescent Carbon Nanodots: Emergent Nanolights. Angewandte Chemie International Edition 49(38), 6726-6744.

Baker, S.N. and Baker, G.A. (2010c) Luminescent carbon nanodots: emergent nanolights. Angew Chem Int Ed Engl 49(38), 6726-6744.

Bamberger, M. and Oswald, R.E. (2012) Impacts of gas drilling on human and animal health. New Solut 22(1), 51-77.

Bao, L.J. and Zeng, E.Y. (2014) Field application of passive sampling techniques for sensing hydrophobic organic contaminants. Trends in Environmental Analytical Chemistry 1, e19-e24.

Baptista, F.R., Belhout, S.A., Giordani, S. and Quinn, S.J. (2015) Recent developments in carbon nanomaterial sensors. Chem Soc Rev 44(13), 4433-4453.

Barahona, F., Turiel, E., Cormack, P.A.G. and Martín-Esteban, A. (2010) Chromatographic performance of molecularly imprinted polymers: Core-shell microspheres by precipitation polymerization and grafted MIP films via inifertermodified silica beads. Journal of Polymer Science Part A: Polymer Chemistry 48(5), 1058-1066. 
Bayati, M., Dai, J., Zambrana, A., Rees, C. and de Cortalezzi, M.F. (2017) Effect of water chemistry on the aggregation and photoluminescence behavior of carbon dots. Journal of Environmental Sciences.

Bosco, F.G., Bache, M., Hwu, E.T., Chen, C.H., Andersen, S.S., Nielsen, K.A., Keller, S.S., Jeppesen, J.O., Hwang, I.S. and Boisen, A. (2012) Statistical analysis of DNT detection using chemically functionalized microcantilever arrays. Sensors Actuat B: Chem 171-172, 1054-1059.

Bourlinos, A.B., Zbořil, R., Petr, J., Bakandritsos, A., Krysmann, M. and Giannelis, E.P. (2011) Luminescent surface quaternized carbon dots. Chemistry of Materials 24(1), 6-8.

Cao, S., Chen, J., Sheng, W., Wu, W., Zhao, Z. and Long, F. (2012) Molecularly Imprinted Sensors. Ge, S.L. and Lunec, S.A.P. (eds), pp. 57-72, Elsevier, Amsterdam.

Caykara, T.C., Bozkaya, U. and Kantoglu, O. (2003) Network structure and swelling behavior of poly(acrylamide/crotonic acid) hydrogels in aqueous salt solutions. Journal of Polymer Science Part B: Polymer Physics 41, 1656-1664.

Cayuela, A., Soriano, M.L., Carrillo-Carrion, C. and Valcarcel, M. (2016) Semiconductor and carbon-based fluorescent nanodots: the need for consistency. Chem Commun (Camb) 52(7), 1311-1326.

Cayuela, A., Soriano, M.L. and Valcárcel, M. (2015) Photoluminescent carbon dot sensor for carboxylated multiwalled carbon nanotube detection in river water. Sensors and Actuators B: Chemical 207, 596-601.

Cecinato, A., Guerriero, E., Balducci, C. and Muto, V. (2014) Use of the PAH fingerprints for identifying pollution sources. Urban Climate.

Chang, H.-Y., Shih, T.-S., Guo, Y.L., Tsai, C.-Y. and Hsu, P.-C. (2004) Sperm function in workers exposed to N,N-dimethylformamide in the synthetic leather industry. Fertil Steril 81(6), 1589-1594.

Chen, C.Y., Wang, C.H. and Chen, A.H. (2011) Recognition of molecularly imprinted polymers for a quaternary alkaloid of berberine. Talanta 84(4), 1038-1046.

Chen, W., Ma, Y., Pan, J., Meng, Z., Pan, G. and Sellergren, B. (2015) Molecularly Imprinted Polymers with Stimuli-Responsive Affinity: Progress and Perspectives. Polymers 7(9), 1689-1715.

Chen, W., Meng, Z., Xue, M. and Shea, K.J. (2016) Molecular imprinted photonic crystal for sensing of biomolecules. Molecular Imprinting 4(1), 1-12. 
Chowdhury, I., Duch, M.C., Mansukhani, N.D., Hersam, M.C. and Bouchard, D. (2013) Colloidal Properties and Stability of Graphene Oxide Nanomaterials in the Aquatic Environment. Environmental Science \& Technology 47(12), 6288-6296.

Christiansen, S., Scholze, M., Axelstad, M., Boberg, J., Kortenkamp, A. and Hass, U. (2008) Combined exposure to anti-androgens causes markedly increased frequencies of hypospadias in the rat. Int J Androl 31(2), 241-248.

Colborn, T., Kwiatkowski, C., Schultz, K. and Bachran, M. (2010) Natural Gas Operations from a Public Health Perspective Hum Ecol Risk Assess 17(5), 10391056.

Colborn, T., Kwiatkowski, C., Schultz, K., Bachran, M. (2011) Natural Gas Operations from a Public Health Perspective. International Journal of Human and Ecological Risk Assessment 17(5), 18.

Committee on Energy and Commerce (2011) Chemicals Used in Hydraulic Fracturing. Representatives, U.S.H.o. (ed).

Cormack, P.A. and Elorza, A.Z. (2004) Molecularly imprinted polymers: synthesis and characterisation. J Chromatogr B Analyt Technol Biomed Life Sci 804(1), 173182.

Creasy, D. (1985) A quantitative study of stage-specific spermatocyte damage following administration of ethylene glycol monomethyl ether in the rat. Experimental and Molecular Pathology 43(3).

Dadvand, P., Parker, J., Bell, M.L., Bonzini, M., Brauer, M., Darrow, L.A., Gehring, U., Glinianaia, S.V., Gouveia, N., Ha, E.H., Leem, J.H., van den Hooven, E.H., Jalaludin, B., Jesdale, B.M., Lepeule, J., Morello-Frosch, R., Morgan, G.G., Pesatori, A.C., Pierik, F.H., Pless-Mulloli, T., Rich, D.Q., Sathyanarayana, S., Seo, J., Slama, R., Strickland, M., Tamburic, L., Wartenberg, D., Nieuwenhuijsen, M.J. and Woodruff, T.J. (2013) Maternal exposure to particulate air pollution and term birth weight: a multi-country evaluation of effect and heterogeneity. Environ Health Perspect 121(3), 267-373.

Dai, J., Dong, X. and de Cortalezzi, M.F. (2017) Molecularly imprinted polymers labeled with amino-functionalized carbon dots for fluorescent determination of 2, 4dinitrotoluene. Microchimica Acta, 1-9.

Davison, W. and Woof, C. (1985) Performance tests for the measurement of $\mathrm{pH}$ with glass electrodes in low ionic strength solutions including natural waters. Analytical Chemistry 57(13), 2567-2570.

Davoodi, D., Hassanzadeh-Khayyat, M., Rezaei, M.A. and Mohajeri, S.A. (2014) Preparation, evaluation and application of diazinon imprinted polymers as the sorbent in molecularly imprinted solid-phase extraction and liquid 
chromatography analysis in cucumber and aqueous samples. Food Chem 158, 421-428.

Demeestere, K., Petrovic, M., Gros, M., Dewulf, J., Van Langenhove, H. and Barcelo, D. (2010) Trace analysis of antidepressants in environmental waters by molecularly imprinted polymer-based solid-phase extraction followed by ultra-performance liquid chromatography coupled to triple quadrupole mass spectrometry. Anal Bioanal Chem 396(2), 825-837.

Deng, S., Yu, G. and Ting, Y.P. (2006) Removal of Humic Acid Using PEI-Modified Fungal Biomass. Separation Science and Technology 41(13), 2989-3002.

Devillers, J., Chezeau, A., Thybaud, E., Poulsen, V., Porcher, J.-M., Graff, L., Vasseur, P., Mouchet, F., Ferrirer, V. and Quiniou, F. (2002) Ecotoxicity of Ethylene Glycol Monobutyl Ether and Its Acetate. Toxicology Mechanisms and Methods 12,9 .

Dhenadhayalan, N. and Lin, K.C. (2015) Chemically induced fluorescence switching of carbon-dots and its multiple logic gate implementation. Sci Rep 5, 10012.

Dickert, F., Lieberzeit, P., Achatz, P., Palfinger, C., Fassnauer, M., Schmid, E., Werther, W. and Horner, G. (2004) QCM array for on-line-monitoring of composting procedures. Analyst 129(5), 432-437.

Dimiev, A.M., Alemany, L.B. and Tour, J.M. (2012) Graphene oxide. Origin of acidity, its instability in water, and a new dynamic structural model. ACS nano 7(1), 576588 .

Ding, C., Zhu, A. and Tian, Y. (2014) Functional Surface Engineering of C-Dots for Fluorescent Biosensing and in Vivo Bioimaging. Accounts Chem Res 47(1), 2030.

Dong, Y., Wang, R., Li, G., Chen, C., Chi, Y. and Chen, G. (2012a) Polyaminefunctionalized carbon quantum dots as fluorescent probes for selective and sensitive detection of copper ions. Anal Chem 84(14), 6220-6224.

Dong, Y., Wang, R., Li, H., Shao, J., Chi, Y., Lin, X. and Chen, G. (2012b) Polyaminefunctionalized carbon quantum dots for chemical sensing. Carbon 50(8), 28102815 .

Dubin, D. (2003) Ion adventure in the heartland: exploring the heart's ionic-molecular microcosm, Cover Publishing Company.

Elimelech, M., Gregory, J. and Jia, X. (2013) Particle deposition and aggregation: measurement, modelling and simulation, Butterworth-Heinemann. 
Esteves da Silva, J.C.G. and Gonçalves, H.M.R. (2011) Analytical and bioanalytical applications of carbon dots. TrAC Trends in Analytical Chemistry 30(8), 13271336.

Fang, Y., Guo, S., Li, D., Zhu, C., Ren, W., Dong, S. and Wang, E. (2011) Easy synthesis and imaging applications of cross-linked green fluorescent hollow carbon nanoparticles. ACS nano 6(1), 400-409.

Feriancikova, L. and Xu, S. (2012) Deposition and remobilization of graphene oxide within saturated sand packs. J Hazard Mater 235-236, 194-200.

Fontenot, B.E., Hunt, L.R., Hildenbrand, Z.L., Carlton Jr, D.D., Oka, H., Walton, J.L., Hopkins, D., Osorio, A., Bjorndal, B., Hu, Q.H. and Schug, K.A. (2013) An Evaluation of Water Quality in Private Drinking Water Wells Near Natural Gas Extraction Sites in the Barnett Shale Formation. Environ Sci Technol.

Gam-Derouich, S., Bourdillon, C., Lakhdar Chaouche, S., Coolen, L., Maitre, A., Mangeney, C. and Schwob, C. (2017) Imprinted Photonic Hydrogels for the Sizeand Shell-Selective Recognition of Nanoparticles. Angew Chem Int Ed Engl.

Gao, R., Kong, X., Su, F., He, X., Chen, L. and Zhang, Y. (2010) Synthesis and evaluation of molecularly imprinted core-shell carbon nanotubes for the determination of triclosan in environmental water samples. J Chromatogr A 1217(52), 8095-8102.

García-Calzón, J.A. and Díaz-García, M.E. (2007) Characterization of binding sites in molecularly imprinted polymers. Sensors and Actuators B: Chemical 123(2), 1180-1194.

Goodpaster, J.V. and McGuffin, V.L. (2001a) Fluorescence Quenching as an Indirect Detection Method for Nitrated Explosives. Analytical Chemistry 73(9), 20042011.

Goodpaster, J.V. and McGuffin, V.L. (2001b) Fluorescence Quenching as an Indirect Detection Method for Nitrated Explosives. Anal. Chem. 73(9), 2004-2011.

Gregory, J. (1975) Interaction of unequal double layers at constant charge. J Colloid Interface Sci 51(1), 44-51.

Gregory, J. (1981) Approximate expressions for retarded van der waals interaction. J Colloid Interface Sci 83(1), 138-145.

Griffete, N., Frederich, H., Maitre, A., Cheimi, M., Ravaine, S. and Mangeney, C. (2011a) Photonic crystal $\mathrm{pH}$ sensor containing a planar defect for fast and enhanced response. Journal of Materials Chemistry 21, 13052-13055. 
Griffete, N., Frederich, H., Maitre, A., Ravaine, S., Chehimi, M.M. and Mangeney, C. (2012) Inverse opals of molecularly imprinted hydrogels for the detection of bisphenol A and pH sensing. Langmuir 28(1), 1005-1012.

Griffete, N., Frederich, H., Maitre, A., Schwob, C., Ravaine, S., Carbonnier, B., Cheimi, M. and Mangeney, C. (2011b) Introduction of a planar defect in a molecularly imprinted photonic crystal sensor for the detection of bisphenol A. Journal of Colloid and Interface Science 364, 18-23.

Hao, T., Wei, X., Nie, Y., Xu, Y., Yan, Y. and Zhou, Z. (2016) An eco-friendly molecularly imprinted fluorescence composite material based on carbon dots for fluorescent detection of 4-nitrophenol. Microchi Acta 183(7), 2197-2203.

Haupt, K. (2003) Peer Reviewed: Molecularly Imprinted Polymers: The Next Generation. Analytical Chemistry 75(17), 376 A-383 A.

Haupt, K. (ed) (2012) Molecular Imprinting, Springer, Berlin.

He, C., Long, Y., Pan, J., Li, K. and Liu, F. (2007) Application of molecularly imprinted polymers to solid-phase extraction of analytes from real samples. Journal of Biochemical and Biophysical Methods 70, 133-150.

Hecker, M., Tyler, C., Hoffmann, M. and Maddix, S. (2002) Plasma biomarkers in fish provide evidence for endocrine modulation in the Elbe River, Germany. Environmental Science and Technology 36(11), 2311-2321.

Henry, O.Y., Cullen, D.C. and Piletsky, S.A. (2005) Optical interrogation of molecularly imprinted polymers and development of MIP sensors: a review. Anal Bioanal Chem 382(4), 947-956.

Holtz, J.H. and Asher, S.A. (1999) Entropic Trapping of Macromolecules by Mesoscopic Periodic Voids in a Polymer Hydrogel. Nature 389, 829-832.

Hsu, P.-C., Chen, P.-C., Ou, C.-M., Chang, H.-Y. and Chang, H.-T. (2013) Extremely high inhibition activity of photoluminescent carbon nanodots toward cancer cells. Journal of Materials Chemistry B 1(13), 1774-1781.

Hsu, P.-C., Shih, Z.-Y., Lee, C.-H. and Chang, H.-T. (2012) Synthesis and analytical applications of photoluminescent carbon nanodots. Green Chemistry 14(4), 917920.

Hu, M.L., Jiang, M., Wang, P., Mei, S.R., Lin, Y.F., Hu, X.Z., Shi, Y., Lu, B. and Dai, K. (2007) Selective solid-phase extraction of tebuconazole in biological and environmental samples using molecularly imprinted polymers. Anal Bioanal Chem 387(3), 1007-1016. 
Hu, S.-L., Niu, K.-Y., Sun, J., Yang, J., Zhao, N.-Q. and Du, X.-W. (2009) One-step synthesis of fluorescent carbon nanoparticles by laser irradiation. Journal of Materials Chemistry 19(4), 484-488.

Hu, X., An, Q., Li, G., Tao, S. and Liu, J. (2006) Imprinted Photonic Polymers for Chiral Recognition. Angewandte Chemie 45, 8145-8148.

Hu, X., Huang, J., Zhang, W., Li, M., Tao, C. and Li, G. (2008a) Photonic Ionic Liquids Polymer for Naked-Eye Detection of Anions. Advanced Materials 20, 4074-4078.

Hu, X., Li, G., Li, M., Huang, J., Li, Y., Gao, Y. and Zhang, Y. (2008b) Ultrasensitive Specific Stimulant Assay Based on Molecularly Imprinted Photonic Hydrogels. Advanced Functional Materials 18(4), 575-583.

Huang, J., Hu, X., Zhang, W., Zhang, Y. and Li, G. (2008) pH and ionic strength responsive photonic polymers fabricated by using colloidal crystal templating. Colloid and Polymer Science 286(1), 113-118.

Jalili, R. and Amjadi, M. (2015) Surface molecular imprinting on silane-functionalized carbon dots for selective recognition of nifedipine. RSC Adv. 5(90), 74084-74090.

Javanbakht, M. and Akbari-Adergani, B. (2012) Molecularly Imprinted Sensors. Ge, S.L. and Lunec, S.A.P. (eds), pp. 247-273, Elsevier, Amsterdam.

Jelinek, R. (2016) Carbon Quantum Dots: Synthesis, Properties and Applications, Springer International Publishing.

Jia, X., Li, J. and Wang, E. (2012) One-pot green synthesis of optically pH-sensitive carbon dots with upconversion luminescence. Nanoscale 4(18), 5572-5575.

Jiang, P., Bertone, J., Hwang, K. and Colvin, V. (1999) Single-crystal colloidal multilayers of controlled thickness. Chem Mater 11(8), 2132-2140.

Johnson, A. and Sumpter, J. (2001) Removal of Endocrine-Disrupting Chemicals in Activated Sludge Treatment Works. Environmental Science and Technology 35(24), 4697-4703.

Johnson-White, B., Zeinali, M., Shaffer, K.M., Patterson, C.H., Charles, P.T. and Markowitz, M.A. (2007) Detection of organics using porphyrin embedded nanoporous organosilicas. Biosensors and Bioelectronics 22(6), 1154-1162.

Kabessa, Y., Eyal, O., Bar-On, O., Korouma, V., Yagur-Kroll, S., Belkin, S. and Agranat, A.J. (2016) Standoff detection of explosives and buried landmines using fluorescent bacterial sensor cells. Biosens Bioelectron 79, 784-788.

Kanekiyo, Y., Naganawa, R. and Tao, H. (2003) pH-responsive molecularly imprinted polymers. Angew Chem Int Ed Engl 42(26), 3014-3016. 
Kanekiyo, Y., Tao, H. and Sellergren, B. (2008) Stimuli-responsive guest binding and releasing by dendritic polymer-based hydrogels. Polymer journal 40(8), 684 .

Karim, K., Breton, F., Rouillon, R., Piletska, E.V., Guerreiro, A., Chianella, I. and Piletsky, S.A. (2005) How to find effective functional monomers for effective molecularly imprinted polymers? Adv Drug Deliv Rev 57(12), 1795-1808.

Kassotis, C.D., Tillitt, D.E., Davis, J.W., Hormann, A.M. and Nagel, S.C. (2014) Estrogen and Androgen Receptor Activities of Hydraulic Fracturing Chemicals and Surface and Ground Water in a Drilling-Dense Region. Endocrinology 155(3), 11 .

Kassotis, C.D., Tillitt, D.E., Lin, C.H., McElroy, J.A. and Nagel, S.C. (2016) EndocrineDisrupting Chemicals and Oil and Natural Gas Operations: Potential Environmental Contamination and Recommendations to Assess Complex Environmental Mixtures. Environ Health Perspect 124(3), 256-264.

Katime, I., Díaz de Apodaca, E., Mendizábal, E. and Puig, J.E. (2000) Acrylic acid/methyl methacrylate hydrogels. I. Effect of composition on mechanical and thermodynamic properties. J Macromol Sci A 37(4), 307-321.

Katz, E. and Willner, I. (2004) Integrated nanoparticle-biomolecule hybrid systems: synthesis, properties, and applications. Angewandte Chemie International Edition 43(45), 6042-6108.

Kempe, H. and Kempe, M. (2010) Influence of salt ions on binding to molecularly imprinted polymers. Anal Bioanal Chem 396(4), 1599-1606.

Kriz, D., Ramström, O. and Mosbach, K. (1997) Peer Reviewed: Molecular Imprinting: New Possibilities for Sensor Technology. Analytical Chemistry 69(11), 345A349A.

Kueseng, P., Noir, M.L., Mattiasson, B., Thavarungkul, P. and Kanatharana, P. (2009) Molecularly imprinted polymer for analysis of trace atrazine herbicide in water. Journal of Environmental Science and Health, Part B 44(8), 772-780.

L. Chen, Xua, S. and Li, J. (2011) Recent advances in molecular imprinting technology: current status, challenges and highlighted applications. Chemical Society Reviews 40, 2922-2942.

Lakowicz, J.R. and Masters, B.R. (2008) Principles of fluorescence spectroscopy. Journal of biomedical optics 13(2), 029901.

Lakshmi, D., Bossi, A., Whitcombe, M.J., Chianella, I., Fowler, S.A., Subrahmanyam, S., Piletska, E.V. and Piletsky, S.A. (2009) Electrochemical sensor for catechol and dopamine based on a catalytic molecularly imprinted polymer-conducting polymer hybrid recognition element. Analytical Chemistry 81(9), 3576-3584. 
Lanphere, J.D., Luth, C.J. and Walker, S.L. (2013) Effects of solution chemistry on the transport of graphene oxide in saturated porous media. Environmental Science \& Technology 47(9), 4255-4261.

Lent, E.M., Crouse, L.C.B., Quinn, M.J. and Wallace, S.M. (2012) Comparison of the Repeated Dose Toxicity of Isomers of Dinitrotoluene. Int J Toxicol 31(2), 143157.

Li, D. and Kaner, R.B. (2008) Graphene-based materials. Nat Nanotechnol 3, 101.

Li, H., He, X., Kang, Z., Huang, H., Liu, Y., Liu, J., Lian, S., Tsang, C.H., Yang, X. and Lee, S.T. (2010a) Water-soluble fluorescent carbon quantum dots and photocatalyst design. Angew Chem Int Ed Engl 49(26), 4430-4434.

Li, H., He, X., Kang, Z., Huang, H., Liu, Y., Liu, J., Lian, S., Tsang, C.H.A., Yang, X. and Lee, S.T. (2010b) Water-soluble fluorescent carbon quantum dots and photocatalyst design. Angewandte Chemie International Edition 49(26), 44304434.

Li, H., Li, Y. and Cheng, J. (2010c) Molecularly Imprinted Silica Nanospheres Embedded CdSe Quantum Dots for Highly Selective and Sensitive Optosensing of Pyrethroids. Chemistry of Materials 22(8), 2451-2457.

Li, H., Zhang, Y., Wang, L., Tian, J. and Sun, X. (2011) Nucleic acid detection using carbon nanoparticles as a fluorescent sensing platform. Chem Commun (Camb) 47(3), 961-963.

Li, S., Ge, Y., Piletsky, S.A. and Lunec, J. (2012) Molecularly imprinted sensors: overview and applications, Elsevier.

Li, X., Rui, M., Song, J., Shen, Z. and Zeng, H. (2015) Carbon and Graphene Quantum Dots for Optoelectronic and Energy Devices: A Review. Adv Funct Mater 25(31), 4929-4947.

Liang, F., Kerpen, K., Kuklya, A. and Telgheder, U. (2012) Fingerprint identification of volatile organic compounds in gasoline contaminated groundwater using gas chromatography differential ion mobility spectrometry. International Journal for Ion Mobility Spectrometry 15(3), 169-177.

Lin, C.H., Lerch, R.N., Garrett, H.E. and George, M.F. (2007) Improved GC-MS/MS Method for Determination of Atrazine and Its Chlorinated Metabolites in Forage Plants-Laboratory and Field Experiments. Communications in soil science and plant analysis 38(13-14), 1753-1773.

Liu, G., Chen, Z., Jiang, X., Feng, D.-Q., Zhao, J., Fan, D. and Wang, W. (2016) In-situ hydrothermal synthesis of molecularly imprinted polymers coated carbon dots for fluorescent detection of bisphenol A. Sensors and Actuat B: Chem 228, 302-307. 
Liu, G., Ding, X., Cao, Y., Zheng, Z. and Peng, Y. (2004) Shape Memory of HydrogenBonded Polymer Network/Poly(ethylene glycol) Complexes. Macromol 37(6), 2228-2232.

Liu, J.M., Lin, L.P., Wang, X.X., Lin, S.Q., Cai, W.L., Zhang, L.H. and Zheng, Z.Y. (2012a) Highly selective and sensitive detection of $\mathrm{Cu} 2+$ with lysine enhancing bovine serum albumin modified-carbon dots fluorescent probe. Analyst 137(11), 2637-2642.

Liu, Y., Liu, C.-y. and Zhang, Z.-y. (2012b) Synthesis of highly luminescent graphitized carbon dots and the application in the Hg $2+$ detection. Applied Surface Science 263, 481-485.

Llop, S., Ballester, F., Estarlich, M., Esplugues, A., Rebagliato, M. and Iniguez, C. (2010) Preterm birth and exposure to air pollutants during pregnancy. Environ Res $110(8), 778-785$.

Long, M., Strand, J., Lassen, P., Krüger, T., Dahllöf, I., Bossi, R., Larsen, M.M., WibergLarsen, P. and Bonefeld-Jørgensen, E.C. (2014) Endocrine-disrupting effects of compounds in Danish streams. Archives of Environmental Contamination and Toxicology 66(1), 1-18.

Lupo, P.J., Symanski, E., Waller, D.K., Chan, W., Langlois, P.H., Canfield, M.A. and Mitchell, L.E. (2011) Maternal exposure to ambient levels of benzene and neural tube defects among offspring: Texas, 1999-2004. Environ Health Perspect 119(3), 397-402.

Lydy, M.J., Landrum, P.F., Oen, A.M., Allinson, M., Smedes, F., Harwood, A.D., Li, H., Maruya, K.A. and Liu, J. (2014) Passive sampling methods for contaminated sediments: state of the science for organic contaminants. Integrated environmental assessment and management 10(2), 167-178.

Mao, X.-J., Zheng, H.-Z., Long, Y.-J., Du, J., Hao, J.-Y., Wang, L.-L. and Zhou, D.-B. (2010a) Study on the fluorescence characteristics of carbon dots. Spectrochimica Acta Part A: Molecular and Biomolecular Spectroscopy 75(2), 553-557.

Mao, X.J., Zheng, H.Z., Long, Y.J., Du, J., Hao, J.Y., Wang, L.L. and Zhou, D.B. (2010b) Study on the fluorescence characteristics of carbon dots. Spectrochim Acta A Mol Biomol Spectrosc 75(2), 553-557.

Mao, Y., Bao, Y., Han, D., Li, F. and Niu, L. (2012) Efficient one-pot synthesis of molecularly imprinted silica nanospheres embedded carbon dots for fluorescent dopamine optosensing. Biosens Bioelectron 38(1), 55-60.

Matthiessen, P. and Johnson, I. (2006) Implications of research on endocrine disruption for the environmental risk assessment, regulation and monitoring of chemicals in the European Union. Environmental Pollution 146, 9-18. 
Maule, A.L., Makey, C.M., Benson, E.B., Burrows, I.J. and Scammell, M.K. (2013) Disclosure of hydraulic fracturing fluid chemical additives: analysis of regulations. New Solut 23(1), 167-187.

McCluskey, A., Holdsworth, C.I. and Bowyer, M.C. (2007a) Molecularly imprinted polymers (MIPs): sensing, an explosive new opportunity? Organic \& Biomolecular Chemistry 5(20), 3233-3244.

McCluskey, A., Holdsworth, C.I. and Bowyer, M.C. (2007b) Molecularly imprinted polymers (MIPs): sensing, an explosive new opportunity? Org Biomol Chem 5(20), 3233-3244.

McKenzie, L.M., Guo, R., Witter, R.Z., Savitz, D.A., Newman, L.S. and Adgate, J.L. (2014) Birth Outcomes and Maternal Residential Proximity to Natural Gas Development in Rural Colorado. Environ Health Perspect.

Mishra, M. and Yagci, Y. (2008) Handbook of vinyl polymers: radical polymerization, process, and technology, CRC press.

Mulabagal, V., Yin, F., John, G.F., Hayworth, J.S. and Clement, T.P. (2013) Chemical fingerprinting of petroleum biomarkers in Deepwater Horizon oil spill samples collected from Alabama shoreline. Marine Pollution Bulletin 70(1-2), 147-154.

Nie, H., Li, M., Li, Q., Liang, S., Tan, Y., Sheng, L., Shi, W. and Zhang, S.X.-A. (2014) Carbon Dots with Continuously Tunable Full-Color Emission and Their Application in Ratiometric pH Sensing. Chemistry of Materials 26(10), 31043112.

Okay, O. (2009) Hydrogel sensors and actuators, pp. 1-14, Springer.

Orem, W., Tatu, C., Varonka, M., Lerch, H., Bates, A., Engle, M., Crosby, L. and McIntosh, J. (2014) Organic substances in produced and formation water from unconventional natural gas extraction in coal and shale. International Journal of Coal Geology 1, 12.

Pan, G., Guo, Q., Cao, C., Yang, H. and Li, B. (2013) Thermo-responsive molecularly imprinted nanogels for specific recognition and controlled release of proteins. Soft Matter 9(14), 3840-3850.

Part, F., Zaba, C., Bixner, O., Zafiu, C., Hann, S., Sinner, E.-K. and Huber-Humer, M. (2016) Traceability of fluorescent engineered nanomaterials and their fate in complex liquid waste matrices. Environmental Pollution 214, 795-805.

Pei, W., Zhu, Y.Y., Zeng, L., Liu, S.X., Wang, X.J. and An, Z.J. (2013) The remote sensing identification of marine oil spill based on oil fingerprinting, pp. 756-765. 
Pelley, A.J. and Tufenkji, N. (2008) Effect of particle size and natural organic matter on the migration of nano-and microscale latex particles in saturated porous media. $\mathrm{J}$ Colloid Interface Sci 321(1), 74-83.

Peng, H., Wang, S., Zhang, Z., Xiong, H., Li, J., Chen, L. and Li, Y. (2012) Molecularly imprinted photonic hydrogels as colorimetric sensors for rapid and label-free detection of vanillin. J Agric Food Chem 60(8), 1921-1928.

Peveler, W.J., Roldan, A., Hollingsworth, N., Porter, M.J. and Parkin, I.P. (2016) Multichannel Detection and Differentiation of Explosives with a Quantum Dot Array. ACS nano 10(1), 1139-1146.

Piletsky, S.A. and Turner, A.P.F. (2008) Optical Biosensors (Second Edition), pp. 543581, Elsevier, Amsterdam.

Qu, S., Wang, X., Lu, Q., Liu, X. and Wang, L. (2012) A Biocompatible Fluorescent Ink Based on Water-Soluble Luminescent Carbon Nanodots. Angewandte Chemie International Edition 51(49), 12215-12218.

Rao, C.e.N.e.R., Sood, A.e.K., Subrahmanyam, K.e.S. and Govindaraj, A. (2009) Graphene: the new two-dimensional nanomaterial. Angewandte Chemie International Edition 48(42), 7752-7777.

Rao, T.P., Prasad, K., Kala, R. and Gladis, J.M. (2007) Biomimetic sensors for toxic pesticides and inorganics based on optoelectronic/electrochemical transducersAn overview. Critical Reviews in Analytical Chemistry 37(3), 191-210.

Reddy, K.R., Khodadoust, A.P. and Darko-Kagya, K. (2014) Transport and Reactivity of Lactate-Modified Nanoscale Iron Particles for Remediation of DNT in Subsurface Soils. J Environ Eng 140(12), 04014042.

S. Wei, Molinelli, A. and Mizaikoff, B. (2006) Molecularly imprinted micro and nanospheres for the selective recognition of 17ß-Estradiol. Biosensor and Bioelectronics 21, 1943-1951.

Saleh, N.B., Pfefferle, L.D. and Elimelech, M. (2010) Influence of biomacromolecules and humic acid on the aggregation kinetics of single-walled carbon nanotubes. Environmental Science \& Technology 44(7), 2412-2418.

Salinas, Y., Martínez-Máñez, R., Marcos, M.D., Sancenón, F., Costero, A.M., Parra, M. and Gil, S. (2012) Optical chemosensors and reagents to detect explosives. Chemical Society Reviews 41(3), 1261-1296.

Sambe, H., Hoshina, K. and Haginaka, J. (2007) Molecularly imprinted polymers for triazine herbicides prepared by multi-step swelling and polymerization method. Their application to the determination of methylthiotriazine herbicides in river water. J Chromatogr A 1152(1-2), 130-137. 
Schneider, C.A., Rasband, W.S. and Eliceiri, K.W. (2012a) NIH Image to ImageJ: 25 years of image analysis. Nat Meth 9(7), 671-675.

Schneider, C.A., Rasband, W.S. and Eliceiri, K.W. (2012b) NIH Image to ImageJ: 25 years of image analysis. Nature methods 9(7), 671-675.

Sellergren, B., Lepistoe, M. and Mosbach, K. (1988) Highly enantioselective and substrate-selective polymers obtained by molecular imprinting utilizing noncovalent interactions. NMR and chromatographic studies on the nature of recognition. Journal of the American Chemical Society 110(17), 5853-5860.

Sergeyeva, T., Piletsky, S., Brovko, A., Slinchenko, E., Sergeeva, L. and El'Skaya, A. (1999) Selective recognition of atrazine by molecularly imprinted polymer membranes. Development of conductometric sensor for herbicides detection. Analytica Chimica Acta 392(2), 105-111.

Shan, X., Chai, L., Ma, J., Qian, Z., Chen, J. and Feng, H. (2014) B-doped carbon quantum dots as a sensitive fluorescence probe for hydrogen peroxide and glucose detection. Analyst 139(10), 2322-2325.

Sharma, A., Jana, T., Kesavamoorthy, R., Shi, L., Virji, M., Finegold, D. and Asher, S. (2004) A General Photonic Crystal Sensing Motif: Creatinine in Bodily Fluids. Journal of the American Chemical Society 126, 2971-2977.

Shen, J., Zhu, Y., Yang, X. and Li, C. (2012a) Graphene quantum dots: emergent nanolights for bioimaging, sensors, catalysis and photovoltaic devices. Chemical Communications 48(31), 3686-3699.

Shen, J., Zhu, Y., Yang, X. and Li, C. (2012b) Graphene quantum dots: emergent nanolights for bioimaging, sensors, catalysis and photovoltaic devices. Chem Commun 48(31), 3686-3699.

Shi, X., Wu, A., Qu, G., Li, R. and Zhang, D. (2007) Development and characterisation of molecularly imprinted polymers based on methacrylic acid for selective recognition of drugs. Biomaterials 28(25), 3741-3749.

Sohoni, P. and Sumpter, J.P. (1998) Several environmental oestrogens are also antiandrogens. Journal of Endocrinology 158, 13.

Song, Y., Feng, D., Shi, W., Li, X. and Ma, H. (2013) Parallel comparative studies on the toxic effects of unmodified CdTe quantum dots, gold nanoparticles, and carbon nanodots on live cells as well as green gram sprouts. Talanta 116, 237-244.

Srivastava, S. and Gajbhiye, N.S. (2011) Carbogenic Nanodots: Photoluminescence and Room-Temperature Ferromagnetism. ChemPhysChem 12(14), 2624-2632.

Stark, G.R. (1965) Reactions of Cyanate with Functional Groups of Proteins. III. Reactions with Amino and Carboxyl Groups*. Biochemistry 4(6), 1030-1036. 
Stöber, W., Fink, A. and Bohn, E. (1968) Controlled growth of monodisperse silica spheres in the micron size range. J colloid interf sci 26(1), 62-69.

Stringer, R.C., Gangopadhyay, S. and Grant, S.A. (2010) Detection of Nitroaromatic Explosives Using a Fluorescent-Labeled Imprinted Polymer. Anal. Chem. 82(10), 4015-4019.

Stuart, M.E., Lapworth, D.J., Thomas, J. and Edwards, L. (2014) Fingerprinting groundwater pollution in catchments with contrasting contaminant sources using microorganic compounds. Science of the Total Environment 468-469, 564-577.

Tan, F., Sun, D., Gao, J., Zhao, Q., Wang, X., Teng, F., Quan, X. and Chen, J. (2013) Preparation of molecularly imprinted polymer nanoparticles for selective removal of fluoroquinolone antibiotics in aqueous solution. J Hazard Mater 244-245, 750757.

Tang, J., Kong, B., Wu, H., Xu, M., Wang, Y., Wang, Y., Zhao, D. and Zheng, G. (2013) Carbon nanodots featuring efficient FRET for real-time monitoring of drug delivery and two-photon imaging. Adv Mater 25(45), 6569-6574.

Tang, Y., Liang, S., Yu, S., Gao, N., Zhang, J., Guo, H. and Wang, Y. (2012) Enhanced adsorption of humic acid on amine functionalized magnetic mesoporous composite microspheres. Colloids and Surfaces A: Physicochemical and Engineering Aspects 406, 61-67.

Tao, H., Yang, K., Ma, Z., Wan, J., Zhang, Y., Kang, Z. and Liu, Z. (2012) In vivo NIR fluorescence imaging, biodistribution, and toxicology of photoluminescent carbon dots produced from carbon nanotubes and graphite. Small 8(2), 281-290.

Tao, Q., Xu, Z., Wang, J., Liu, F., Wan, H. and Zheng, S. (2010) Adsorption of humic acid to aminopropyl functionalized SBA-15. Microporous and Mesoporous Materials 131(1-3), 177-185.

Tchounwou, P.B., Newsome, C., Glass, K., Centeno, J.A., Leszczynski, J., Bryant, J., Okoh, J., Ishaque, A. and Brower, M. (2003) Environmental toxicology and health effects associated with dinitrotoluene exposure. Rev Environ Health 18(3), 203-229.

Thacker, J.B., Carlton, D.D., Hildenbrand, Z.L., Kadjo, A.F. and Schug, K.A. (2015) Chemical analysis of wastewater from unconventional drilling operations. Water 7(4), 1568-1579.

Thayer, K.A. and Belcher, S.M. (2011) Mechanisms of Action of Bisphenol A and other Biochemical/Molecular Interactions, World Health Organization.

The Shale Gas Subcommittee of the Secretary of Energy Advisory Board (2011) The SEAB Shale Gas Production Subcommittee Ninety-Day Report. 
Thomas, P. and Budiantara, L. (1995) Reproductive Life History Stages Sensitive to Oil and Naphthalene in Atlantic Croaker. Marine Environmental Research 39, 4.

Toal, S.J. and Trogler, W.C. (2006) Polymer sensors for nitroaromatic explosives detection. Journal of Materials Chemistry 16(28), 2871.

Troester, M., Brauch, H.-J. and Hofmann, T. (2016) Vulnerability of drinking water supplies to engineered nanoparticles. Water Res 96, 255-279.

Umpleby, R.J., 2nd, Baxter, S.C., Rampey, A.M., Rushton, G.T., Chen, Y. and Shimizu, K.D. (2004) Characterization of the heterogeneous binding site affinity distributions in molecularly imprinted polymers. J Chromatogr B Analyt Technol Biomed Life Sci 804(1), 141-149.

USEPA (2012) Study of the Potential Impacts of Hydraulic Fracturing on Drinking Water Resources: Progress Report, EPA 601/R-12/11, US Environmental Protection Agency.

Uzun, L. and Turner, A.P. (2016) Molecularly-imprinted polymer sensors: realising their potential. Biosens Bioelectron 76, 131-144.

Valero-Navarro, A., Salinas-Castillo, A., Fernandez-Sanchez, J.F., Segura-Carretero, A., Mallavia, R. and Fernandez-Gutierrez, A. (2009) The development of a MIPoptosensor for the detection of monoamine naphthalenes in drinking water. Biosens Bioelectron 24(7), 2305-2311.

Vasapollo, G., Sole, R.D., Mergola, L., Lazzoi, M.R., Scardino, A., Scorrano, S. and Mele, G. (2011) Molecularly imprinted polymers: present and future prospective. International journal of molecular sciences 12(9), 5908-5945.

Vázquez-González, M. and Carrillo-Carrion, C. (2014) Analytical strategies based on quantum dots for heavy metal ions detection. Journal of biomedical optics 19(10), 101503-101503.

Verwey, E.J.W. (1947) Theory of the Stability of Lyophobic Colloids. The Journal of Physical and Colloid Chemistry 51(3), 631-636.

Wagner, S., Gondikas, A., Neubauer, E., Hofmann, T. and von der Kammer, F. (2014) Spot the difference: engineered and natural nanoparticles in the environment-release, behavior, and fate. Angew Chem Int Ed Engl 53(46), 12398-12419.

Wahba, M.E.K., El-Enany, N. and Belal, F. (2015) Application of the Stern-Volmer equation for studying the spectrofluorimetric quenching reaction of eosin with clindamycin hydrochloride in its pure form and pharmaceutical preparations. Anal Method 7(24), 10445-10451. 
Wang, C., Xu, Z. and Zhang, C. (2015a) Polyethyleneimine-Functionalized Fluorescent Carbon Dots: Water Stability, pH Sensing, and Cellular Imaging. ChemNanoMat $1(2), 122-127$.

Wang, E., Sun, D., Li, H., Sun, X., Liu, J., Ren, Z. and Yan, S. (2016) High efficiency organosilicon-containing polymer sensors for the detection of trinitrotoluene and dinitrotoluene. J Mater Chem C 4(28), 6756-6760.

Wang, H., Yi, J., Velado, D., Yu, Y. and Zhou, S. (2015b) Immobilization of Carbon Dots in Molecularly Imprinted Microgels for Optical Sensing of Glucose at Physiological pH. ACS Appl Mater Interfac 7(29), 15735-15745.

Wang, L.Q., Lin, F.Y. and Yu, L.P. (2012a) A molecularly imprinted photonic polymer sensor with high selectivity for tetracyclines analysis in food. Analyst 137(15), 3502-3509.

Wang, S., Ge, L., Song, X., Yan, M., Ge, S., Yu, J. and Zeng, F. (2012b) Simple and covalent fabrication of a paper device and its application in sensitive chemiluminescence immunoassay. Analyst 137(16), 3821-3827.

Wang, X., Qu, K., Xu, B., Ren, J. and Qu, X. (2011a) Microwave assisted one-step green synthesis of cell-permeable multicolor photoluminescent carbon dots without surface passivation reagents. Journal of Materials Chemistry 21(8), 2445-2450.

Wang, X., Qu, K., Xu, B., Ren, J. and Qu, X. (2011b) Microwave assisted one-step green synthesis of cell-permeable multicolor photoluminescent carbon dots without surface passivation reagents. J Mater Chem 21(8), 2445-2450.

Wang, Y., Anilkumar, P., Cao, L., Liu, J.-H., Luo, P., Tackett II, K., Sahu, S., Wang, P., Wang, X. and Sun, Y.-P. (2011c) Carbon Dots of different composition and surface functionalization: Cytotoxicity issues relevant to fluorescence cell imaging. Experimental Biology and Medicine 236, 1231-1238.

Wang, Y., Anilkumar, P., Cao, L., Liu, J.-H., Luo, P.G., Tackett, K.N., Sahu, S., Wang, P., Wang, X. and Sun, Y.-P. (2011d) Carbon dots of different composition and surface functionalization: cytotoxicity issues relevant to fluorescence cell imaging. Exp Bio Med 236(11), 1231-1238.

Wang, Y. and Hu, A. (2014a) Carbon quantum dots: synthesis, properties and applications. Journal of Materials Chemistry C 2(34), 6921-6939.

Wang, Y. and Hu, A. (2014b) Carbon quantum dots: synthesis, properties and applications. J Mater Chem C 2(34), 6921.

Wang, Y., Li, C.Y., Mo, H.L., Sun, Y.Z., Chen, Y.M. and Wan, P.Y. (2013a) Extraction of chemical fingerprint in food industry wastewater, pp. 1484-1490. 
Wang, Y., Wang, S., Ge, S., Wang, S., Yan, M., Zang, D. and Yu, J. (2013b) Facile and sensitive paper-based chemiluminescence DNA biosensor using carbon dots dotted nanoporous gold signal amplification label. Analytical Methods 5(5), 1328-1336.

Warner, N.R., Jackson, R.B., Darrah, T., Osborn, S.G., Down, A., Zhao, K., White, A. and Vengosh, A. (2012) Geochemical evidence for possible natural migration of Marcellus Formation brine to shallow aquifers in Pennsylvania. Proceedings of the National Academy of Sciences 109(30), 11961-11966.

Watabe, Y., Hosoya, K., Tanaka, N., Kubo, T., Kondo, T. and Morita, M. (2005) Novel surface modified molecularly imprinted polymer focused on the removal of interference in environmental water samples for chromatographic determination. Journal of Chromatography A 1073(1-2), 363-370.

Wei, W., Xu, C., Ren, J., Xu, B. and Qu, X. (2012) Sensing metal ions with ion selectivity of a crown ether and fluorescence resonance energy transfer between carbon dots and graphene. Chem Commun (Camb) 48(9), 1284-1286.

Wen, Y., Chen, L., Li, J., Ma, Y., Xu, S., Zhang, Z., Niu, Z. and Choo, J. (2012) Molecularly imprinted matrix solid-phase dispersion coupled to micellar electrokinetic chromatography for simultaneous determination of triazines in soil, fruit, and vegetable samples. Electrophoresis 33(15), 2454-2463.

Whitcombe, M.J., Martin, L. and Vulfson, E.N. (1998) Predicting the selectivity of imprinted polymers. Chromatographia 47(7-8), 457-464.

Wu, E., Peng, Y., Zhang, X., Bai, J., Song, Y., He, H., Fan, L., Qu, X., Gao, Z., Liu, Y. and Ning, B. (2017) Ultrasensitive Sensing Material Based on Opal Photonic Crystal for Label-Free Monitoring of Transferrin. ACS Appl Mater Interfaces 9(7), 5778-5783.

Wu, H.-G., Ju, X.-J., Xie, R., Liu, Y.-M., Deng, J.-G., Niu, C.H. and Chu, L.-Y. (2010) A novel ion-imprinted hydrogel for recognition of potassium ions with rapid response. Polymers for Advanced Technologies, n/a-n/a.

Wu, Z., Tao, C., Lin, C., Shen, D. and Li, G. (2008a) Label-Free Colorimetric Detection of Trace Atrazine in Aqueous Solution by Using Molecularly Imprinted Photonic Polymers. Chemistry. A European Journal 14, 11358-11368.

Wu, Z., Tao, C.A., Lin, C., Shen, D. and Li, G. (2008b) Label-free colorimetric detection of trace atrazine in aqueous solution by using molecularly imprinted photonic polymers. Chemistry 14(36), 11358-11368.

Wulff, G. (2001) Enzyme-like Catalysis by Molecularly Imprinted Polymers. Chemical Reviews 102(1), 1-28. 
Wulff, G. (2002) Enzyme-like Catalysis by Molecularly Imprinted Polymers. Chem Rev 102(1), 1-28.

Xu, D., Zhu, W., Jiang, Y., Li, X., Li, W., Cui, J., Yin, J. and Li, G. (2012) Rational design of molecularly imprinted photonic films assisted by chemometrics. Journal of Materials Chemistry 22(32), 16572-16581.

$\mathrm{Xu}$, J. and Jing, N. (2012) Effects of 2,4-dinitrotoluene exposure on enzyme activity, energy reserves and condition factors in common carp (Cyprinus carpio). J. Hazard. Mater. 203-204, 299-307.

$\mathrm{Xu}, \mathrm{S} ., \mathrm{Lu}, \mathrm{H}$. and Chen, L. (2014) Double water compatible molecularly imprinted polymers applied as solid-phase extraction sorbent for selective preconcentration and determination of triazines in complicated water samples. J Chromatogr A 1350, 23-29.

Xu, S., Lu, H., Li, J., Song, X., Wang, A., Chen, L. and Han, S. (2013) Dummy molecularly imprinted polymers-capped CdTe quantum dots for the fluorescent sensing of 2,4,6-trinitrotoluene. ACS Appl Mater Interfac 5(16), 8146-8154.

Yan, M. (2004) Molecularly imprinted materials: science and technology, CRC press.

Yang, M., Han, A., Duan, J., Li, Z., Lai, Y. and Zhan, J. (2012a) Magnetic nanoparticles and quantum dots co-loaded imprinted matrix for pentachlorophenol. J Hazard Mater 237-238, 63-70.

Yang, Y., Cui, J., Zheng, M., Hu, C., Tan, S., Xiao, Y., Yang, Q. and Liu, Y. (2012b) One-step synthesis of amino-functionalized fluorescent carbon nanoparticles by hydrothermal carbonization of chitosan. Chem Commun 48(3), 380-382.

Yin, Y.M., Chen, Y.P., Wang, X.F., Liu, Y., Liu, H.L. and Xie, M.X. (2012) Dummy molecularly imprinted polymers on silica particles for selective solid-phase extraction of tetrabromobisphenol A from water samples. J Chromatogr A 1220, $7-13$.

Yu, C., Li, X., Zeng, F., Zheng, F. and Wu, S. (2013) Carbon-dot-based ratiometric fluorescent sensor for detecting hydrogen sulfide in aqueous media and inside live cells. Chem Commun (Camb) 49(4), 403-405.

Zhang, K., Zhou, H., Mei, Q., Wang, S., Guan, G., Liu, R., Zhang, J. and Zhang, Z. (2011a) Instant visual detection of trinitrotoluene particulates on various surfaces by ratiometric fluorescence of dual-emission quantum dots hybrid. Journal of the American Chemical Society 133(22), 8424-8427.

Zhang, K., Zhou, H., Mei, Q., Wang, S., Guan, G., Liu, R., Zhang, J. and Zhang, Z. (2011b) Instant Visual Detection of Trinitrotoluene Particulates on Various Surfaces by Ratiometric Fluorescence of Dual-Emission Quantum Dots Hybrid. J Am Chem Soc 133(22), 8424-8427. 
Zhang, W., He, X.W., Chen, Y., Li, W.Y. and Zhang, Y.K. (2011c) Composite of CdTe quantum dots and molecularly imprinted polymer as a sensing material for cytochrome c. Biosens Bioelectron 26(5), 2553-2558.

Zhang, X. and Bai, R. (2003) Mechanisms and kinetics of humic acid adsorption onto chitosan-coated granules. J Colloid Interface Sci 264(1), 30-38.

Zheng, M., Liu, S., Li, J., Qu, D., Zhao, H., Guan, X., Hu, X., Xie, Z., Jing, X. and Sun, Z. (2014a) Integrating oxaliplatin with highly luminescent carbon dots: an unprecedented theranostic agent for personalized medicine. Adv Mater 26(21), 3554-3560.

Zheng, M., Liu, S., Li, J., Qu, D., Zhao, H., Guan, X., Hu, X., Xie, Z., Jing, X. and Sun, Z. (2014b) Integrating Oxaliplatin with Highly Luminescent Carbon Dots: An Unprecedented Theranostic Agent for Personalized Medicine. Adv Mater 26(21), 3554-3560.

Zheng, M., Xie, Z., Qu, D., Li, D., Du, P., Jing, X. and Sun, Z. (2013) On-off-on fluorescent carbon dot nanosensor for recognition of chromium(VI) and ascorbic acid based on the inner filter effect. ACS Appl Mater Interfaces 5(24), 1324213247.

Zhu L, Chen L and L, X. (2003) Application of a Molecularly Imprinted Polymer for the Effective Recognition of Different Anti-Epidermal Growth Factor Receptor Inhibitors. Analytical Chemistry 75(23), 6381-6387.

Zhu, S., Meng, Q., Wang, L., Zhang, J., Song, Y., Jin, H., Zhang, K., Sun, H., Wang, H. and Yang, B. (2013) Highly photoluminescent carbon dots for multicolor patterning, sensors, and bioimaging. Angew Chem Int Ed Engl 52(14), 3953-3957.

Zhu, S., Song, Y., Zhao, X., Shao, J., Zhang, J. and Yang, B. (2015) The photoluminescence mechanism in carbon dots (graphene quantum dots, carbon nanodots, and polymer dots): current state and future perspective. Nano Research $8(2), 355-381$.

Zuo, P., Gao, J., Peng, J., Liu, J., Zhao, M., Zhao, J., Zuo, P. and He, H. (2015) A sol-gel based molecular imprint incorporating carbon dots for fluorometric determination of nicotinic acid. Microchim Acta 183(1), 329-336. 


\section{VITA}

Jingjing Dai was born on September 19, 1988 in Liaoning, China. From 20072011, she studied Environmental Engineering at Nankai University. She attended the University of Missouri in August 2012, and received her master degree in July 2014 and Ph.D degree in December 2017, respectively. She will start her career as a water engineer at Arcadis. 